\title{
Existence locale et effet régularisant précisés pour des équations non linéaires de type Schrödinger
}

\author{
Pierre-Yves Bienaimé
}

Résumé. In this paper, we consider the Cauchy problem in the usual Sobolev spaces for some nonlinear equations of the form

$$
\left\{\begin{array}{l}
\partial_{t} u=i \mathscr{L} u+F\left(u, \nabla_{x} u, \bar{u}, \nabla_{x} \bar{u}\right), \quad t \in \mathbb{R}, x \in \mathbb{R}^{n}, \\
u(x, 0)=u_{0}(x) \in H^{s}\left(\mathbb{R}^{n}\right),
\end{array}\right.
$$

that is, equations which are of Schrödinger type. We study the local existence and the smoothing effect of the solutions, following C.E. Kenig, G. Ponce and L. Vega, and extend some of their results.

The nonlinearity $F$ is a smooth function which vanishes to the 3 rd order at 0 and the operator $\mathscr{L}$ has the form $\mathscr{L}=\sum_{j \leq k} \partial_{x_{j}}^{2}-\sum_{j>k} \partial_{x_{j}}^{2}$. It extends the Laplace operator but is not elliptic in general.

We prove the local existence, the uniqueness and the smoothing effect given any $u_{0} \in H^{s}\left(\mathbb{R}^{n}\right)$ with $s>n / 2+3$. The proof follows the same plan as that of Kenig, Ponce and Vega, [5]. We improve the estimates by using the paradifferential calculus of J.-M. Bony.

\section{Introduction}

On considère le problème de Cauchy suivant :

$$
\left\{\begin{array}{l}
\partial_{t} u=i \mathscr{L} u+F\left(u, \nabla_{x} u, \bar{u}, \nabla_{x} \bar{u}\right), \quad t \in \mathbb{R}, x \in \mathbb{R}^{n}, \\
u(x, 0)=u_{0}(x) \in H^{s}\left(\mathbb{R}^{n}\right)
\end{array}\right.
$$

où $u=u(x, t)$ est une fonction à valeurs complexes, $\bar{u}$ sa fonction conjuguée, $\nabla_{x} u=\left(\partial_{x_{1}} u, \ldots, \partial_{x_{n}} u\right), F(u, v, \bar{u}, \bar{v})$ une fonction $C^{\infty}$ de $\mathbb{C} \times \mathbb{C}^{n} \times \mathbb{C} \times \mathbb{C}^{n}$ dans $\mathbb{C}$ et

$$
\mathscr{L}=\sum_{j \leq k} \partial_{x_{j}}^{2}-\sum_{j>k} \partial_{x_{j}}^{2}, \quad k \in\{1, \ldots, n\},
$$

Mathematics Subject Classification (2010): Primary 35, 47, 46; Secondary 35Q, 35S, 35E, 35M, 47B, 47F, 47G, 47S, 46T.

Keywords: Nonlinear generalized Schrödinger type equations, Sobolev spaces, pseudo-differential and para-differential operators, Bony formula, Cauchy problem, local existence, smoothing effect. 
est un opérateur différentiel qui, bien qu'il puisse être égal au laplacien, n'est pas elliptique en général. Il s'agit donc d'équations non linéaires de type Schrödinger, et on étudie, dans ce travail, le caractère bien posé dans les espaces de Sobolev habituels $H^{s}\left(\mathbb{R}^{n}\right)$ du problème de Cauchy associé, ainsi que l'effet régularisant des solutions, en suivant les travaux de C. E. Kenig, G. Ponce et L. Vega.

Le résultat principal de cet article est le suivant :

Théorème 1.1. On suppose la fonction $F$ nulle à l'ordre 3 en 0 , autrement dit, $F$ et ses dérivées partielles jusqu'à l'ordre 2 sont nulles en 0 . Alors, pour tout $s>n / 2+3$ et toute fonction $u_{0} \in H^{s}\left(\mathbb{R}^{n}\right)$, il existe un réel $T>0$ tel que le problème de Cauchy (1.1) possède une solution unique $u \in E_{T}$ où $E_{T}$ désigne l'ensemble des fonctions $u \in C\left([-T, T] ; H^{s}\left(\mathbb{R}^{n}\right)\right)$ telles que

$$
\left\|\mid J^{s+1 / 2} u\right\|_{T} \stackrel{\text { def }}{=} \sup _{\mu \in \mathbb{Z}^{n}}\left(\int_{-T}^{T} \int_{\mathbb{R}^{n}}\left|\left\langle x-x_{\mu}\right\rangle^{-2} J^{s+1 / 2} u(x, t)\right|^{2} d x d t\right)^{1 / 2}<\infty,
$$

avec $J$ l'opérateur $(1-\Delta)^{1 / 2}$ et $\Delta=\sum_{k=1}^{k=n} \partial_{x_{k}}^{2}$.

De plus, pour un borné $B$ de $H^{s}$ de données initiales, les solutions associées ont un même temps d'existence $T_{B}$, et, l'application qui à $u_{0} \in B$ associe $u \in E_{T_{B}}$ est uniformément continue.

Dans [5], C. E. Kenig, G. Ponce et L. Vega démontrent ce théorème dans le cas où $F$ est un polynôme de valuation $v \geq 3$ et $s>s_{0}$ où $s_{0}$ est assez grand. Ces auteurs ne se sont pas intéressés à la valeur du $s_{0}$, mais en reprenant leur démonstration, on peut voir qu'il est supérieur à $n / 2+10 n+1$. Par ailleurs, ils ont aussi étudié le cas où $F$ est de valuation 2 , mais il semble que dans ce cas il soit nécessaire de travailler dans les espaces de Sobolev à poids. En ce qui nous concerne, nous traiterons ce cas à part dans un prochain article. On peut remarquer que dans ce cas, dans [5], il est annoncé que la régularité $s_{0}$ utilisée est supérieure à $40 n+62$.

Le problème de Cauchy (1.1) a beaucoup été étudié dans les années 1990 surtout quand $\mathscr{L}=\Delta$, autrement dit, dans le cas de l'équation de Schrödinger. Nous renvoyons le lecteur intéressé à l'introduction très instructive de [5] et à la bibliographie de cet article. Quand au cas $\mathscr{L} \neq \Delta$, cas moins bien connu, son étude est motivée par l'existence d'un certain nombre d'équations provenant des applications et qui sont de ce type. Par exemple, les equations de type Ishimori (modèle de spin-1 en cristallographie) :

$$
i \partial_{t} u+\partial_{x_{1}}^{2} u-\partial_{x_{2}}^{2} u=\frac{2 \bar{u}}{1+|u|^{2}}\left(\left(\partial_{x_{1}} u\right)^{2}-\left(\partial_{x_{2}} u\right)^{2}\right)
$$

ou les systèmes type Davey-Stewartson :

$$
\left\{\begin{array}{l}
i \partial_{t} u+\partial_{x_{1}}^{2} u-\partial_{x_{2}}^{2} u=u|u|^{2}+\partial_{x_{2}} v u \\
\Delta v\left(x_{1}, x_{2}\right)=-\partial_{x_{2}}\left(|u|^{2}\right)
\end{array}\right.
$$

qui décrivent l'évolution de paquets d'ondes en eau d'une profondeur finie, même si ce dernier système ne se met pas exactement sous la forme (1.1). Pour plus de détails, voir [11], [7]. 
Notons aussi que L. T'Joen, [13], a généralisé les résultats de [5] au cas d'un opérateur $\mathscr{L}$ à coefficients variables et elliptique, sous l'hypothèse géométrique de non captance, sans préciser l'indice $s_{0}$, tandis que, dans [7], les auteurs traitent le cas non semi-linéaire, autrement dit, $\mathscr{L}$ est à coefficients variables et dépendant de $u$, en faisant certaines hypothèses, notamment d'ellipticité et de non captance, et avec un indice $s_{0}$ assez grand. Enfin, dans [12], J. Szeftel traite aussi le cas à coefficients variables et démontre un résultat d'effet régularisant microlocal en admettant l'existence des solutions et avec une non linéarité de la forme $F(u, \bar{u})$.

Dans cet article, l'effet régularisant est précisé car, dans [5], l'effet régularisant est obtenu pour

$$
\left\|\mid J^{s+1 / 2} u\right\| \|_{T, 1} \stackrel{\text { def }}{=} \sup _{\mu \in \mathbb{Z}^{n}}\left(\int_{-T}^{T} \int_{Q_{\mu}}\left|J^{s+1 / 2} u(x, t)\right|^{2} d x d t\right)^{1 / 2},
$$

où $Q_{\mu}$ est le cube $\mu+[0,1]^{n}$ de grande diagonale de longueur $d_{n}$ indépendante de $\mu$. Or, on a

$$
\left\|J^{s+1 / 2} u\right\|_{T, 1} \leq\left\langle d_{n}\right\rangle^{4}\left\|\mid J^{s+1 / 2} u\right\|_{T},
$$

où $d_{n}$ est la longueur de la grande diagonale de $Q_{0}$. Ce nouvel effet régularisant est donc plus précis.

Le théorème 1.1 est démontré pour les régularités $s>s_{0}$ avec $s_{0}=n / 2+3$. Naturellement, la question de l'optimalité de ce $s_{0}$ se pose. Il se trouve que nous ne disposons pas de contre-exemple pour le cas $s \leq s_{0}$ et nous ne savons pas si $s_{0}$ est optimal. Bien entendu, la valeur de ce $s_{0}$ est le résultat des techniques de démonstration utilisées. A titre de comparaison, on peut noter que, dans [6], on obtient le résultat du théorème 1.1 pour $s \geq 1 / 2+3$ en dimension 1 et pour $s \geq n+5 / 2$ en dimension $n \geq 2$ dans le cas où $\mathscr{L}=\Delta, F$ polynôme de valuation 3 et $\left\|u_{0}\right\|_{s_{0}}$ assez petite. Cependant, il n'est pas difficile de vérifier qu'avec une non linéarité de la forme $F(u, \bar{u})$, on a le résultat d'existence et d'unicité pour $s>n / 2$. Pour plus de détails, on pourra, par exemple, aller voir [4]. De plus, toujours dans [4], on montre que dans certains cas particuliers de (1.1), on a existence et unicité pour $s>n / 2+1$.

Donnons maintenant une idée sur la démonstration du théorème 1.1. Celle-ci suit le schéma de celle de [5] et passe bien entendu par une linéarisation. Cependant, pour obtenir des estimations plus précises, nous linéarisons l'équation (1.1) en utilisant la paralinéarisation de J.-M. Bony, [1], et un certain calcul symbolique paradifférentiel. L'utilisation de ce type de calcul permet essentiellement de supprimer les pertes de régularité liées aux commutations.

Ainsi, au lieu de linéariser de manière classique pour se ramener à étudier l'équation linéaire

$$
\partial_{t} u=i \mathscr{L} u+a_{1} u+a_{2} \bar{u}+b_{1} \nabla_{x} u+b_{2} \nabla_{x} \bar{u}+f(x, t),
$$

nous linéarisons selon la méthode de Bony pour se ramener à étudier l'équation suivante, qui est aussi linéaire :

$$
\partial_{t} u=i \mathscr{L} u+T_{a_{1}} u+T_{a_{2}} \bar{u}+T_{b_{1}} \nabla_{x} u+T_{b_{2}} \nabla_{x} \bar{u}+f(x, t),
$$


où $T_{a_{1}}, T_{a_{2}}, T_{b_{1}}$ et $T_{b_{2}}$ sont des opérateurs paradifférentiels et plus précisément des opérateurs de paramultiplication (voir section 2). Cette équation est souvent dite paralinéaire.

Pour expliquer l'hypothèse d'annulation faite sur $F$, rappelons que, dans le cas où $\mathscr{L}=\Delta$ et $b_{2}=0$, pour que le problème de Cauchy linéaire associé à (1.2) soit bien posé, une condition nécessaire portant sur le coefficient $b_{1}(x)$ a été démontrée :

$$
\sup _{x \in \mathbb{R}^{n}, \omega \in \mathbb{S}^{n-1}, R>0}\left|\mathscr{I} \int_{0}^{R} b_{1}(x+r \omega) \cdot \omega d r\right|<\infty .
$$

Voir [16], [10]. Cette condition est évidemment vérifiée si $b_{1}$ est réel. Elle est aussi vérifiée si $b_{1}=v w$ ou avw, pour $v, w \in H^{s}\left(\mathbb{R}^{n}\right), s>n / 2$ et $a \in L^{\infty}\left(\mathbb{R}^{n}\right)$, comme on peut le voir aisément en appliquant le théorème de trace. Comme le coefficient $b_{1}$ est une dérivée de $F$, ceci justifie l'hypothèse faite sur $F$ et explique aussi pourquoi l'on est contraint de travailler dans des espaces à poids si $F$ s'annule seulement à l'ordre 2 en 0.

La démonstration du théorème 1.1 consiste en grande partie à établir des estimations convenables sur la solution du problème de Cauchy associé à l'équation paralinéaire (1.3). On commence par traiter le cas d'un intervalle $[0, T]$, la démonstration étant la même pour obtenir les résultats sur l'intervalle $[-T, 0]$ sachant que, par exemple, on a

$$
\begin{aligned}
\sup _{\mu \in \mathbb{Z}^{n}} & \left(\int_{-T}^{T} \int_{\mathbb{R}^{n}}\left|\left\langle x-x_{\mu}\right\rangle^{-2} J^{s+1 / 2} u(x, t)\right|^{2} d x d t\right)^{1 / 2} \\
& \leq \sup _{\mu \in \mathbb{Z}^{n}}\left(\int_{-T}^{0} \int_{\mathbb{R}^{n}}\left|\left\langle x-x_{\mu}\right\rangle^{-2} J^{s+1 / 2} u(x, t)\right|^{2} d x d t\right)^{1 / 2}+\left|\left\|J^{s+1 / 2} u \mid\right\|_{T}\right.
\end{aligned}
$$

avec, à partir d'ici,

$$
\left\|\mid J^{s+1 / 2} u\right\| \|_{T}=\sup _{\mu \in \mathbb{Z}^{n}}\left(\int_{0}^{T} \int_{\mathbb{R}^{n}}\left|\left\langle x-x_{\mu}\right\rangle^{-2} J^{s+1 / 2} u(x, t)\right|^{2} d x d t\right)^{1 / 2} .
$$

Pour ce faire et suivant [5], on construit un opérateur pseudodifférentiel $\mathbf{C}$ d'ordre 0 et possédant de bonnes propriétés, notamment inversible et de symbole $\mathbf{c}(x, \xi)$ réel et pair en $\xi$. Cela permet, par estimation de $\|\mathbf{C} u\|_{0}$, d'obtenir des estimations d'énergie sur les solutions de (1.3) du type

$$
\begin{gathered}
\sup _{0 \leq t \leq T}\|u(t)\|_{s} \leq A\left(\left\|u_{0}\right\|_{s}+\int_{[0, T]}\|f(t)\|_{s} d t\right), \\
\|\| J^{s+1 / 2} u(t)\|\|_{T} \leq A\left(\left\|u_{0}\right\|_{s}+\int_{[0, T]}\|f(t)\|_{s} d t\right), \\
\sup _{0 \leq t \leq T}\|u(t)\|_{s} \leq A\left(\left\|u_{0}\right\|_{s}+\mathcal{N}_{T}\left(J^{s-1 / 2} f\right)\right), \\
\|\| J^{s+1 / 2} u\|\|_{T} \leq A\left(\left\|u_{0}\right\|_{s}+\mathcal{N}_{T}\left(J^{s-1 / 2} f\right)\right),
\end{gathered}
$$


avec

$\mathcal{N}_{T}(u)=\sup _{\mu \in \mathbb{Z}^{n}}\left(\int_{0}^{T} \int_{\mathbb{R}^{n}}\left|\left\langle x-x_{\mu}\right\rangle^{2} u(x, t)\right|^{2} d x d t\right)^{1 / 2}=\left\|\left\langle x-x_{\mu}\right\rangle^{2} u\right\|_{l_{\mu}^{\infty}\left(L^{2}\left(\mathbb{R}^{n} \times[0, T]\right)\right)}$.

On remarque que l'on peut aussi obtenir les estimations ci-dessus avec

$$
\left\|\mid J^{-1 / 2} f\right\|_{T}^{\prime}=\sum_{\mu \in \mathbb{Z}^{n}}\left(\int_{0}^{T} \int_{Q_{\mu}}\left|J^{-1 / 2} f\right|^{2} d x d t\right)^{1 / 2}
$$

au lieu de $\mathcal{N}_{T}\left(J^{-1 / 2} f\right)$, comme dans [5].

On met alors l'équation (1.1) sous la forme (1.3) avec $a_{1}=\partial_{u} F\left(z_{0}\right), a_{2}=$ $\partial_{\bar{u}} F\left(z_{0}\right), b_{1}=\nabla_{v} F\left(z_{0}\right), b_{2}=\nabla_{\bar{v}} F\left(z_{0}\right)$ où $z_{0}=\left(u_{0}, v_{0}, \bar{u}_{0}, \bar{v}_{0}\right), v=\nabla u$ et un terme non linéaire $f(x, t)=R\left(u, \nabla_{x} u, \bar{u}, \nabla_{x} \bar{u}\right)$. Dans la suite, $W(t) u_{0}$ désigne la solution de (1.3) avec $f=0$ telle que $W(0) u_{0}=u_{0}$. On détermine un espace métrique complet dans lequel on prouve que l'opérateur

$$
\Upsilon u=W(t) u_{0}+\int_{0}^{T} W\left(t-t^{\prime}\right) R\left(u, \nabla_{x} u, \bar{u}, \nabla_{x} \bar{u}\right) d t^{\prime}
$$

admet un unique point fixe pour un $T$ assez petit. Ce dernier résultat s'obtient en utilisant les estimations (1.5) et (1.6). Pour estimer la non-linéarité, on utilise la propriété d'algèbre de $H^{s}$ pour $s>n / 2$.

Pour prouver (1.5) et (1.6), on estime la norme $L^{2}$ de $\mathbf{C} u$ où $u$ est une solution de (1.3) en écrivant de manière classique

$$
\partial_{t}\|\mathbf{C} u\|_{0}^{2}=\left\langle\partial_{t} \mathbf{C} u, \mathbf{C} u\right\rangle+\left\langle\mathbf{C} u, \partial_{t} \mathbf{C} u\right\rangle,
$$

ce qui permet d'obtenir que

$$
\begin{aligned}
\left\|\mathbf{C} u\left(T_{0}\right)\right\|_{0}^{2} \leq & \left\|\mathbf{C} u_{0}\right\|_{0}^{2}+\left|2 \mathscr{R} \int_{0}^{T_{0}}\left\langle i[\mathbf{C}, \mathscr{L}] u+\mathbf{C} T_{i \mathscr{I}\left(b_{1}\right)} \nabla u, \mathbf{C} u\right\rangle d t\right| \\
& +\left|2 \mathscr{R} \int_{0}^{T_{0}}\left\langle\mathbf{C} T_{b_{2}} \nabla \bar{u}, \mathbf{C} u\right\rangle d t\right|+\left|2 \mathscr{R} \int_{0}^{T_{0}}\langle\mathbf{C} u, \mathbf{C} f\rangle d t\right| \\
& +A_{c} T_{0} \sup _{\left[0, T_{0}\right]}\|u(t)\|_{0}^{2}+\frac{A_{c}}{R}\left\|J^{1 / 2} u\right\|_{T_{0}}^{2}
\end{aligned}
$$

où $A_{c}$ est une constante qui dépend des semi-normes d'ordre $M$ (suffisamment grand) des symboles de $\mathbf{C}$ et $T_{b_{1}}$.

La difficulté de l'équation (1.3) semblant venir du coefficient $b_{1}$, on cherche à choisir $\mathbf{C}$ pour que l'opérateur

$$
i[\mathbf{C}, \mathscr{L}]+\mathbf{C} T_{i \mathscr{I}\left(b_{1}\right)} \cdot \nabla
$$

soit relativement petit, en un sens que l'on précisera, l'idéal étant qu'il soit continu sur $L^{2}$, ce qui nous ramène à étudier son symbole principal

$$
-2 \widetilde{\xi} \cdot \nabla_{x} \mathbf{c}(x, \xi)-\mathbf{c}(x, \xi) \mathscr{I}\left(\widetilde{b}_{1}(x, \xi)\right) \cdot \xi
$$

où $\widetilde{b}_{1}(x, \xi)$ est tel que $T_{i \mathscr{I}\left(b_{1}\right)}=i \mathscr{I}\left(\widetilde{b}_{1}(x, D)\right)$ et $\widetilde{\xi}=\left(-\xi_{1}, \ldots,-\xi_{k}, \xi_{k+1}, \ldots, \xi_{n}\right)$. 
Pour assurer plus ou moins l'inversibilité de $\mathbf{C}$, on cherche son symbole sous la forme $\mathbf{c}(x, \xi)=\exp (\gamma(x, \xi))$. Pour construire $\gamma$, on commence par décomposer $b_{1}$ suivant les cubes $Q_{\mu}$ en écrivant que

$$
b_{1}(x)=\sum_{\mu \in \mathbb{Z}^{n}} \alpha_{1, \mu} \varphi_{1, \mu}(x),
$$

avec $\left(\alpha_{1, \mu}\right) \in l^{1}, \operatorname{supp}\left(\varphi_{1, \mu}\right) \subset 2 Q_{\mu}$ et $\left\|\varphi_{1, \mu}\right\|_{C} \varrho \leq 1$ où $C^{\varrho}$ désigne la classe de Hölder si $\varrho$ n'est pas entier et l'espace des fonctions $\varrho$ fois dérivables à dérivées bornées sinon. Les calculs nous amènent ensuite à résoudre l'équation aux dérivées partielles suivante, d'inconnue $\eta_{\mu}$,

$$
-2 \widetilde{\xi} \cdot \nabla_{x} \eta_{\mu}(x, \xi)-\mathscr{I}\left(\widetilde{\varphi}_{1, \mu}(x, \xi)\right) \cdot \xi=0,
$$

où le symbole $\widetilde{\varphi}_{1, \mu}$ est défini par $T_{\varphi_{1, \mu}}=\widetilde{\varphi}_{1, \mu}(x, D)$. On symmétrise alors en $\xi$ la solution obtenue, puis on la tronque de manière convenable pour obtenir un symbole $\gamma_{\mu}$ d'ordre 0 . On pose enfin

$$
\gamma(x, \xi)=\sum_{\mu \in \mathbb{Z}^{n}} \alpha_{1, \mu} \gamma_{\mu}(x, \xi)
$$

Le symbole $\gamma$ ainsi défini est d'ordre 0 et, même si l'expression

$$
-2 \widetilde{\xi} \cdot \nabla_{x} \gamma(x, \xi)-\mathscr{I}\left(\widetilde{b}_{1}(x, \xi)\right) \cdot \xi
$$

n'est pas d'ordre 0, elle est petite en un sens convenable.

On remarque que, par construction, pour tout $i \in\{1,2\}, b_{i}, \varphi_{i, \mu}$ et $\mathbf{c}$ ont la régularité de $\nabla u_{0}$.

Pour estimer l'autre terme qui pose problème, $\left\langle\mathbf{C} T_{b_{2}} \nabla_{x} \bar{u}, \mathbf{C} u\right\rangle$, on utilise des commutateurs et les propriétés de $\mathbf{C}=\mathbf{c}(x, D)$, notamment que $\mathbf{c}$ est pair et réel en $\xi$, ce qui donne $\mathbf{C} \bar{u}=\overline{\mathbf{C} u}$, et que $\left\langle\nabla_{x} \bar{u}, u\right\rangle=0$.

Les inégalités (1.6) et (1.8) donnent l'effet régularisant. Les inégalités (1.7) et surtout (1.8) sont centrales car elles permettent d'estimer la non linéarité d'ordre 1 dans un espace approprié pour pouvoir appliquer un théorème de point fixe. L'effet régularisant s'obtient en utilisant le lemme de Doi, l'inégalité de Gårding et la décomposition de $b_{1}, b_{2}, a_{1}$ et $a_{2}$ suivant les $Q_{\mu}$, autrement dit, en suivant là encore la méthode utilisée dans [5] et en l'adaptant au cadre plus général des résultats énoncés dans cet article.

Je tiens à remercier A. Boulkhemair pour ses idées et ses conseils avisés.

\section{Notations, définitions et résultats préliminaires}

Dans tout l'article, toute constante qui ne dépend que de la dimension de l'espace $n$ et de la régularité $\varrho$ de $\nabla_{x} u_{0}$ est notée $A$ pour simplifier.

Il n'est pas nécessaire de lire toute cette partie pour comprendre la suite de l'article car ici sont rappelés des notations et des résultats généraux classiques que nous utiliserons plus tard et, notamment, quelques résultats sur les opérateurs pseudodifférentiels et paradifférentiels. 
En revanche, de nombreux lemmes sont démontrés par des techniques qui sont réutilisées dans les démonstrations des résultats principaux et dont les détails ne sont pas rappelés ensuite.

Quelques notations utilisées dans cet article :

- $J^{s}=(1-\Delta)^{s / 2}, \quad \Delta=\sum_{k=1}^{n} \partial_{k}^{2}$ étant le Laplacien,

$-|\alpha|=\sum_{j=1}^{j=n} \alpha_{j}$ si $\alpha \in \mathbb{N}^{n}$,

- $\Delta F=\left(\Delta F_{1}, \ldots, \Delta F_{n}\right)$ et $\nabla F=\left(\nabla F_{1}, \ldots, \nabla F_{n}\right)$ si $F=\left(F_{1}, \ldots, F_{n}\right)$,

- $\mathscr{S}\left(\mathbb{R}^{n}\right)$ désigne l'espace de Schwartz,

- $\widehat{u}$ ou $\mathscr{F}(u)$ désigne la transformée de Fourier de $u$,

- Si $s \in \mathbb{R}, H^{s}\left(\mathbb{R}^{n}\right)=\left\{u \in \mathscr{S}^{\prime}\left(\mathbb{R}^{n}\right) ;\left(1+|\xi|^{2}\right)^{s / 2} \widehat{u} \in L^{2}\left(\mathbb{R}^{n}\right)\right\}$ désigne l'espace de Sobolev, $\|u\|_{H^{s}\left(\mathbb{R}^{n}\right)}=\|u\|_{s}$.

- Classes de symboles de Hörmander $:$ Si $m \in \mathbb{R}$ et $\gamma, \delta \in[0,1]$,

$$
S_{\gamma, \delta}^{m}=\left\{a \in C^{\infty}\left(\mathbb{R}^{2 n}\right) ; \forall \alpha, \beta \in \mathbb{N}^{n},\left|\partial_{x}^{\alpha} \partial_{\xi}^{\beta} a(x, \xi)\right| \leq A_{\alpha, \beta}\langle\xi\rangle^{-\gamma|\beta|+\delta|\alpha|+m}\right\} .
$$

- Si $\varrho$ est un réel positif, $C^{\varrho}\left(\mathbb{R}^{n}\right)$ désignera la classe de Hölder, autrement dit, $u$ est dans $C^{\varrho}\left(\mathbb{R}^{n}\right)$ si $u \in C^{[\varrho]}\left(\mathbb{R}^{n}\right)$ et si

$$
\begin{gathered}
\forall \alpha \in \mathbb{N}^{n},|\alpha| \leq[\varrho], \quad \partial^{\alpha} u \in L^{\infty}\left(\mathbb{R}^{n}\right), \\
\text { et } \exists C \in \mathbb{R}, \forall(x, y) \in \mathbb{R}^{n} \times \mathbb{R}^{n},\left|\partial^{\alpha} u(x)-\partial^{\alpha} u(y)\right| \leq C|x-y|^{\varrho-[\varrho]} .
\end{gathered}
$$

- $O p S$ désignera l'ensemble des opérateurs pseudodifférentiels dont le symbole appartient à la classe $S$.

- Si $Q_{\mu}=\mu+[0,1]^{n}, \mu \in \mathbb{Z}^{n}$, on pose

$$
\begin{aligned}
\|\| u \|_{T} & =\sup _{\mu \in \mathbb{Z}^{n}}\left(\int_{0}^{T} \int_{\mathbb{R}^{n}}\left|\left\langle x-x_{\mu}\right\rangle^{-2} u(x, t)\right|^{2} d x d t\right)^{1 / 2}, \\
\mathcal{N}_{T}(u) & =\sup _{\mu \in \mathbb{Z}^{n}}\left(\int_{0}^{T} \int_{\mathbb{R}^{n}}\left|\left\langle x-x_{\mu}\right\rangle^{2} u(x, t)\right|^{2} d x d t\right)^{1 / 2} \\
& =\left\|\left\langle x-x_{\mu}\right\rangle^{2} u\right\|_{l_{\mu}^{\infty}\left(L^{2}\left(\mathbb{R}^{n} \times[0, T]\right)\right) .}
\end{aligned}
$$

L'énoncé suivant rappelle et résume le calcul pseudodifférentiel associé aux classes de symboles de Hörmander $S_{\gamma, \delta}^{m}$ :

Théorème 2.1 (Calcul pseudodifférentiel). Soient $a \in S_{\gamma, \delta}^{m}$ et $b \in S_{\gamma, \delta}^{m^{\prime}}$ avec $m, m^{\prime} \in \mathbb{R}$, et $0 \leq \delta<\gamma \leq 1$ ou $0 \leq \delta \leq \gamma<1$. Alors :

(i) On a $a(x, D) b(x, D)=c(x, D)$ avec $c \in S_{\gamma, \delta}^{m+m^{\prime}}$. De plus,

$$
\begin{aligned}
c(x, \xi) & =O s \iint e^{-i y \cdot \eta} a(x, \xi+\eta) b(x+y, \xi) \frac{d y d \eta}{(2 \pi)^{n}} \\
& =\sum_{|\nu|<N} \frac{1}{\nu !} \partial_{\xi}^{\nu} a(x, \xi) D_{x}^{\nu} b(x, \xi)+\sum_{|\nu|=N} \frac{1}{\nu !} \int_{0}^{1}(1-\theta)^{N-1} r_{\nu, \theta}(x, \xi) d \theta,
\end{aligned}
$$


où

$$
r_{\nu, \theta}(x, \xi)=O s \iint e^{-i y \cdot \eta} \partial_{\xi}^{\nu} a(x, \xi+\theta \eta) \partial_{x}^{\nu} b(x+y, \xi) \frac{d y d \eta}{(2 \pi)^{n}} .
$$

Enfin, les semi-normes $S_{\gamma, \delta}^{m+m^{\prime}}$ de $r_{\nu, \theta}$ sont bornées par des produits de semi-normes de $\partial_{\xi}^{\nu} a, \partial_{x}^{\nu} b$ uniformément en $\theta \in[0,1]$.

(ii) On a aussi $a(x, D)^{*}=a^{*}(x, D)$ avec $a^{*} \in S_{\gamma, \delta}^{m}$ et

$$
a^{*}(x, \xi)=\sum_{|\nu|<N} \frac{1}{\nu !} \partial_{\xi}^{\nu} \overline{a(x, \xi)}+\sum_{|\nu|=N} \frac{1}{\nu !} \int_{0}^{1}(1-\theta)^{N-1} r_{\nu, \theta}^{*}(x, \xi) d \theta
$$

où

$$
r_{\nu, \theta}^{*}(x, \xi)=O s \iint e^{-i y \cdot \eta} \partial_{\xi}^{\nu} \partial_{x}^{\nu} \overline{a(x+y, \xi+\theta \eta)} \frac{d y d \eta}{(2 \pi)^{n}} .
$$

De plus, les semi-normes $S_{\gamma, \delta}^{m}$ de $r_{\nu, \theta}^{*}$ sont bornées par des produits de semi-normes de $\partial_{\xi}^{\nu} \partial_{x}^{\nu} \bar{a}$ uniformément en $\theta \in[0,1]$.

Preuve : Voir, par exemple, [14].

Lemme 2.2. Soient $\delta$ et $\gamma$ tels que $0 \leq \delta<\gamma \leq 1$ ou $0 \leq \delta \leq \gamma<1$. Soient a et b tels que $a \in S_{\gamma, \delta}^{m}$ et $b \in S_{\gamma, \delta}^{m^{\prime}}$ alors, pour tout $\nu \in \mathbb{N}^{n}, r_{\nu, \theta} \in S_{\gamma, \delta}^{m+m^{\prime}}$ et les $S_{\gamma, \delta}^{m^{\prime}+m}$ semi-normes de $r_{\nu, \theta}$ sont bornées par des produits de semi-normes de $\partial_{\xi}^{\nu} a, \partial_{x}^{\nu} b$ uniformément en $\theta \in[0,1]$.

Théorème 2.3 (Calderón-Vaillancourt). Soit $a: \mathbb{R}^{n} \times \mathbb{R}^{n} \rightarrow \mathbb{C}$ une fonction bornée et $0 \leq \delta<1$. Alors, l'opérateur $a(x, D)$ est continu de $L^{2}\left(\mathbb{R}^{n}\right)$ dans $L^{2}\left(\mathbb{R}^{n}\right)$ si l'une des conditions suivantes est vérifiée :

(i) Il existe une constante $C>0$ telle que, pour tous multi-indices $\alpha$ et $\beta$ tels que $|\alpha|+|\beta| \leq n+1$ et tout $(x, \xi) \in \mathbb{R}^{2 n}, \quad\left|\partial_{x}^{\alpha} \partial_{\xi}^{\beta} a(x, \xi)\right| \leq C\langle\xi\rangle^{\delta(|\alpha|-|\beta|)}$.

(ii) L'inégalité précédente est vérifiée pour tous les multi-indices $\alpha$ et $\beta$ tels que $|\alpha| \leq N$ et $|\beta| \leq N$, où $N$ est un entier tel que $N>n / 2$.

Preuve : Voir [2].

Remarque. Pour tout réel $m \geq 0$, et, pour tout $\delta$ et $\gamma$ tel que $0 \leq \delta \leq \gamma<1$ ou $0 \leq \delta<\gamma \leq 1, S_{\gamma, \delta}^{m} \subset S_{\delta, \delta}^{m}$

Corollaire 2.4. Si $a \in S_{\gamma, \delta}^{0}, 0 \leq \delta \leq \gamma<1$ ou $0 \leq \delta<\gamma \leq 1$ alors

$$
\|\mid a(x, D) f\|_{T} \leq A\|\| f \|_{T} \quad \text { et } \quad \mathcal{N}_{T}(a(x, D) f) \leq A \mathcal{N}_{T}(f) .
$$

Preuve : On a

$$
\|\mid a(x, D) f\|_{T}=\sup _{\mu}\left(\int_{0}^{T} \int_{\mathbb{R}^{n}}\left|\left\langle x-x_{\mu}\right\rangle^{-2} a(x, D) u\right|^{2} d x d t\right)^{1 / 2}
$$

or

$$
a(x, D) u=a(x, D)\left\langle x-x_{\mu}\right\rangle^{2}\left\langle x-x_{\mu}\right\rangle^{-2} u,
$$


et le symbole de l'opérateur $a(x, D)\left\langle x-x_{\mu}\right\rangle^{2}$ est

$$
\sum_{|\nu| \leq 2} \partial_{\xi}^{\nu} a(x, \xi) D_{x}^{\nu}\left(\left\langle x-x_{\mu}\right\rangle^{2}\right)
$$

et donc

$$
\left\langle x-x_{\mu}\right\rangle^{-2} a(x, D)\left\langle x-x_{\mu}\right\rangle^{2} \in S_{\gamma, \delta}^{0} .
$$

Le théorème de Calderón-Vaillancourt donne alors le résultat. Prouvons ensuite la deuxième inégalité. On a

$$
\mathcal{N}_{T}(f)=\sup _{\mu}\left(\int_{0}^{T} \int_{\mathbb{R}^{n}}\left|\left\langle x-x_{\mu}\right\rangle^{2} a(x, D) u\right|^{2} d x d t\right)^{1 / 2} .
$$

L'opérateur $a(x, D)\left\langle x-x_{\mu}\right\rangle^{-2}$ a pour symbole

$$
r(x, \xi)=\int_{\mathbb{R}^{n}} \int_{\mathbb{R}^{n}} e^{i y \cdot \eta}\left\langle x+y-x_{\mu}\right\rangle^{-2} a(x, \xi+\eta) d y d \eta .
$$

Par intégrations par parties en $y$ et $\eta$, puis en utilisant l'inégalité de Peetre

$$
\left\langle x+y-x_{\mu}\right\rangle^{-2-N} \leq A_{N}\left\langle x-x_{\mu}\right\rangle^{-2-N}\langle y\rangle^{N},
$$

on prouve que

$$
\left\langle x-x_{\mu}\right\rangle^{2} r(x, \xi) \in S_{\gamma, \delta}^{0} .
$$

Ce qui termine la démonstration de ce corollaire.

Lemme 2.5. Pour tout réel $s$, tout $N>n / 2$, il existe une constante $C>0$ telle que, pour tout $v \in H^{s}$,

$$
\left\|\langle x-\mu\rangle^{-N} v\right\|_{s} \in l_{\mu}^{2} \quad \text { et } \quad\|\|\langle x-\mu\rangle^{-N} v\left\|_{s}\right\|_{l_{\mu}^{2}} \leq C\|v\|_{s} .
$$

Preuve : Posons $c_{\mu}(x, D)=\langle D\rangle^{s}\langle x-\mu\rangle^{-N}$. D'après le théorème 2.1, on a

$$
c_{\mu}(x, \xi)=O s \int e^{-i y \cdot \eta}\langle\xi+\eta\rangle^{s}\langle x+y-\mu\rangle^{-N} \frac{d y d \eta}{(2 \pi)^{n}} .
$$

On remarque que l'on peut écrire $\langle x-\mu\rangle^{N} c_{\mu}(x, \xi)=c(x-\mu, \xi)$ où

$$
c(x, \xi)=\langle x\rangle^{N} \int e^{-i y \cdot \eta}\langle\xi+\eta\rangle^{s}\langle x+y\rangle^{-N} \frac{d y d \eta}{(2 \pi)^{n}} .
$$

Supposons d'abord que $c \in S_{1,0}^{s}$. On a alors

$$
\begin{aligned}
& \sum_{\mu}\left\|c_{\mu}(x, D) v\right\|_{0}^{2}=\sum_{\mu} \int \frac{|c(x-\mu, D) v(x)|^{2}}{\langle x-\mu\rangle^{2 N}} d x \\
& \sum_{\mu}\left\|c_{\mu}(x, D) v\right\|_{0}^{2} \leq C_{1} \sum_{\mu} \int \frac{\langle x-E(x)\rangle^{2 N}}{\langle E(x)-\mu\rangle^{2 N}}|c(x-\mu, D) v(x)|^{2} d x,
\end{aligned}
$$


où $E(x)$ est le vecteur formé par les parties entières des coordonnées de $x$ et où on a utilisé l'inégalité de Peetre. D'où,

$$
\begin{aligned}
& \sum_{\mu}\left\|c_{\mu}(x, D) v\right\|_{0}^{2} \leq C_{2} \sum_{\mu} \frac{1}{\langle\mu\rangle^{2 N}}\left\|c(x, D) \tau_{-\mu} v\right\|_{0}^{2} \\
& \sum_{\mu}\left\|c_{\mu}(x, D) v\right\|_{0}^{2} \leq C_{3} \sum_{\mu} \frac{1}{\langle\mu\rangle^{2 N}}\left\|\tau_{-\mu} v\right\|_{s}^{2}=C^{2}\|v\|_{s}^{2},
\end{aligned}
$$

puisque $2 N>n, c \in S_{1,0}^{s}$, la norme $H^{s}$ est invariante par translation.

Reste à vérifier que $c \in S_{1,0}^{s}$. L'argument est en fait classique : Par intégrations par parties, on peut écrire $\langle x\rangle^{-N} c(x, \xi)$ comme une combinaison linéaire finie d'intégrales de la forme

$$
\int e^{-i y \cdot \eta} \partial_{\eta}^{\alpha}\langle\xi+\eta\rangle^{s} \partial_{\eta}^{\beta}\langle\eta\rangle^{-N_{1}}\langle y\rangle^{-N_{2}} J_{y}^{N_{1}}\langle x+y\rangle^{-N} d y d \eta
$$

où les entiers pairs $N_{1}, N_{2}$ sont assez grands et $|\alpha|+|\beta| \leq N_{2}$, intégrales que l'on peut estimer, grâce à l'inégalité de Peetre, par

$$
C \int\langle\xi\rangle^{s}\langle\eta\rangle^{-N_{1}+|s|}\langle y\rangle^{-N_{2}+N}\langle x\rangle^{-N} d y d \eta=C^{\prime}\langle\xi\rangle^{s}\langle x\rangle^{-N}
$$

si $N_{1}>n+|s|$ et $N_{2}>n+N$; ce qui prouve que $|c(x, \xi)| \leq C\langle\xi\rangle^{s}$. Les dérivées partielles de $c$ se traitent de la même manière.

Théorème 2.6. Soit $\left(u_{j}\right)$ une suite de fonctions $C^{\infty}$ dans $\mathbb{R}^{n}$. On suppose qu'il existe $s>0$ et $m \in \mathbb{N}$ tels que $m>s,\left(2^{j s}\left\|u_{j}\right\|_{0}\right)_{j} \in l^{2}$ et $\left(2^{j(s-m)}\left\|\partial^{\alpha} u_{j}\right\|_{0}\right)_{j} \in l^{2}$ pour tout $\alpha \in \mathbb{N}^{n}$ tel que $|\alpha|=m$. Alors, $u=\sum_{j} u_{j}$ est dans $H^{s}\left(\mathbb{R}^{n}\right)$ et

$$
\|u\|_{s}^{2} \leq A \sup _{|\alpha| \in\{0, m\}} \sum_{j=0}^{\infty} 2^{2 j(s-|\alpha|)}\left\|u_{j}\right\|_{0}^{2}
$$

où $A$ est une constante indépendante des $u_{j}$.

Preuve : voir [8], [14].

Théorème 2.7. Soit $\left(u_{j}\right)$ une suite de fonctions $C^{\infty}$ dans $\mathbb{R}^{n}$. On suppose qu'il existe un compact $\Gamma \subset \mathbb{R}^{n} \backslash\{0\}$ tel que $s p\left(u_{j}\right) \subset 2^{j} \Gamma$ et un nombre réel $s$ tel que $\left(2^{j s}\left\|u_{j}\right\|_{0}\right)_{j} \in l^{2}$. Alors, $u=\sum_{j} u_{j}$ est dans $H^{s}\left(\mathbb{R}^{n}\right)$ et $\|u\|_{s}^{2} \leq A \sum_{j=0}^{\infty} 2^{2 j s}\left\|u_{j}\right\|_{0}^{2}$, où la constante $A$ est indépendante des $u_{j}$.

Opérateurs paradifférentiels : On rappelle maintenant quelques résultats sur les opérateurs paradifférentiels.

On définit la classe $\Sigma_{\varrho}^{m}$ où $m \in \mathbb{R}$ et $\varrho>0$ comme la classe des symboles $a(x, \xi)$, continus sur $\mathbb{R}^{n} \times \mathbb{R}^{n}, C^{\infty}$ en $\xi$ et $C^{\varrho}$ en $x$, et plus précisément tels que

$$
\forall \alpha \in \mathbb{N}^{n}, \quad\left|\partial_{\xi}^{\alpha} a(x, \xi)\right|\langle\xi\rangle^{-m+|\alpha|} \in C^{\varrho}\left(\mathbb{R}^{n} \times \mathbb{R}^{n}\right) .
$$


A un symbole $a$ dans $\Sigma_{\varrho}^{m}$, J.-M. Bony associe l'opérateur paradifférentiel $T_{a}$ défini par

$$
\widehat{T_{a} u}(\xi)=(2 \pi)^{-n} \int_{\mathbb{R}^{n}} \chi(\xi-\eta, \eta) \mathscr{F}_{1}(a)(\xi-\eta, \eta) \widehat{u}(\eta) d \eta,
$$

où $\chi$ est une paratroncature, c'est à dire une fonction dans $C^{\infty}\left(\mathbb{R}^{n} \times \mathbb{R}^{n}\right)$ vérifiant les deux propriétés suivantes :

(i) $\exists h>0, \varepsilon>0, \varepsilon^{\prime}>0$, avec $\varepsilon^{\prime}<\varepsilon<1$ et $\chi(\xi, \eta)=\left\{\begin{array}{l}0 \text { si }|\xi| \geq \varepsilon|\eta|, \\ 1 \text { si }|\xi| \leq \varepsilon^{\prime}|\eta| \text { et }|\eta| \geq h \text {. }\end{array}\right.$

(ii) $\forall \alpha \in \mathbb{N}^{2 n}, \exists A_{\alpha}>0, \forall \zeta \in \mathbb{R}^{2 n},\langle\zeta\rangle^{|\alpha|}\left|\partial^{\alpha} \chi(\zeta)\right| \leq A_{\alpha}$.

On peut aussi écrire $T_{a}=\widetilde{a}(x, D)$ où $\widetilde{a}$ est un symbole dans la classe $S_{1,1}^{m}$. Cependant, les opérateurs paradifférentiels opèrent bien dans les espaces de Sobolev de la manière habituelle. En effet, on a le

Théorème 2.8. (i) Pour tout s réel, $T_{a}$ est continu de $H^{s}\left(\mathbb{R}^{n}\right)$ dans $H^{s-m}\left(\mathbb{R}^{n}\right)$.

(ii) $\mathrm{Si}$, dans la définition de $T_{a}$, on modifie la paratroncature, alors l'opérateur erreur (ou différence) est continu de $H^{s}\left(\mathbb{R}^{n}\right)$ dans $H^{s-m+\varrho}\left(\mathbb{R}^{n}\right)$ pour tout réel $s$.

Preuve : Voir [1], [9] ou [14].

La seconde partie du théorème précédent montre que la dépendance de $T_{a}$ par rapport à la paratroncature est de moindre importance. Elle explique aussi pourquoi les restes dans la théorie paradifférentielle sont seulement $\varrho$-régularisants.

Remarquons aussi qu'un choix possible de la paratroncature que nous utiliserons dans la suite est donné par

$$
\chi(\xi, \eta)=\chi_{1}(\xi /|\eta|)\left(1-\psi_{1}(\eta)\right),
$$

où $\psi_{1}, \chi_{1} \in C^{\infty}\left(\mathbb{R}^{n}\right), \psi_{1}$ vaut 1 au voisinage de 0 et est à support dans $B(0, h)$, et $\chi_{1}$ est à support dans $B(0, \varepsilon)$ et vaut 1 sur $B\left(0, \varepsilon^{\prime}\right)$, où $\varepsilon$ et $\varepsilon^{\prime}$ sont deux réels tels que $0<\varepsilon^{\prime}<\varepsilon<1$.

Les opérateurs paradifférentiels permettent d'écrire la formule de linéarisation de J.-M. Bony.

Théorème 2.9 (Formule de Bony). Pour toutes fonctions réelles $u_{1}, \ldots, u_{m} \in$ $H^{n / 2+\varrho}\left(\mathbb{R}^{n}\right), \varrho>0$, et toute fonction $F$ de $m$ variables réelles, $C^{\infty}$ et nulle en 0 , on a

$$
F\left(u_{1}, \ldots, u_{m}\right)=\sum_{i=1}^{i=m} T_{\partial_{u_{i}} F} u_{i}+r, \quad \text { avec } r \in H^{n / 2+2 \varrho}\left(\mathbb{R}^{n}\right) .
$$

Preuve : Voir [1]. Cf. aussi [9], [8].

Le reste $r$ dans cette formule dépend évidemment de $\left(u_{1}, \ldots, u_{m}\right)$. Le résultat qui suit étudie cette dépendance et montre que $r$ est une fonction localement lipschitzienne de $\left(u_{1}, \ldots, u_{m}\right)$. Plus précisément : 
Théorème 2.10. Si $u=\left(u_{1}, \ldots, u_{m}\right) \in H^{s}\left(\mathbb{R}^{n}, \mathbb{R}^{m}\right), s=n / 2+\varrho$, $\varrho>0$, on désigne par $r(u)$ le reste obtenu dans la formule de Bony ci dessus. Pour tous $u, v \in H^{s}\left(\mathbb{R}^{n}, \mathbb{R}^{m}\right)$, on a alors

$$
\|r(u)-r(v)\|_{s+\varrho} \leq \theta\left(\|u\|_{s},\|v\|_{s}\right)\|u-v\|_{s},
$$

où $\theta\left(\|u\|_{s},\|v\|_{s}\right)$ est bornée si $u$ et $v$ sont dans un borné de $H^{s}\left(\mathbb{R}^{n}, \mathbb{R}^{m}\right)$.

Preuve : On reprend la méthode utilisée dans l'article [8] pour démontrer la formule de Bony, méthode basée sur l'analyse dyadique. Pour tout $k \geq 0$, on pose $\varphi_{k}(\xi)=$ $\varphi_{0}\left(2^{-k} \xi\right)$ avec $\varphi_{0}(\xi)=\varphi(\xi / 2)-\varphi(\xi)$, où $\varphi=\varphi_{-1} \in \mathscr{D}\left(\mathbb{R}^{n}\right)$ est telle que $\operatorname{supp}(\varphi) \subset$ $B(0,1)$ et $\varphi=1$ sur $B(0,1 / 2)$. On a donc une partition dyadique de l'unité, $1=$ $\sum_{k \geq-1} \varphi_{k}$, ce qui permet d'écrire la décomposition dyadique $u=\sum_{k \geq-1} \varphi_{k}(D) u$ pour toute distribution tempérée $u$. Les $\varphi_{k}(D) u$ sont les termes dyadiques de $u$.

On suppose $m=1$. Les $u_{k}$ désignent les termes dyadiques de $u$ et les $S_{k}(u)$ les sommes partielles jusqu'à $k$ des termes dyadiques de $u$, de sorte que $u_{k}=$ $S_{k}(u)-S_{k-1}(u)$.

On peut écrire

$$
F(u)=F\left(u_{-1}\right)+\sum_{k=0}^{\infty} F\left(S_{k}(u)\right)-F\left(S_{k-1}(u)\right)=F\left(S_{1}(u)\right)+\sum_{k=2}^{\infty} u_{k} r_{k}(u)+T_{F^{\prime}(u)} u
$$

où

$r_{k}(u)=\int_{0}^{1} F^{\prime}\left(S_{k-1}(u)+t u_{k}\right) d t-S_{k-3}\left(F^{\prime}(u)\right) \quad$ et $\quad T_{F^{\prime}(u)} u=\sum_{k=2}^{\infty} u_{k} S_{k-3}\left(F^{\prime}(u)\right)$.

Notons ici que $T_{F^{\prime}(u)}$ est bien un opérateur paradifférentiel, en fait de paramultiplication : il est associé au symbole $F^{\prime}(u(x))$ et à la paratroncature particulière $\chi(\xi, \eta)=\sum_{k \geq 2} \sum_{l \leq k-3} \varphi_{l}(\xi-\eta) \varphi_{k}(\eta)$. Il en résulte l'expression suivante de $r(u)$ :

$$
r(u)=F\left(S_{1}(u)\right)+\sum_{k=2}^{\infty} u_{k} r_{k}(u) .
$$

Notons aussi qu'il est bien connu que l'application $u \mapsto F(u)$ envoie $H^{s}\left(\mathbb{R}^{n}\right)$ dans lui-même $(s>n / 2)$ et qu'elle est bornée sur les bornés, ce qui implique facilement le caractère localement lipschitzien de cette application. D'ailleurs, ceci rend l'étude du terme $F\left(S_{1}(u)\right)$ triviale, terme qui sera donc ignoré dans la suite de cette démonstration.

On peut donc écrire, pour $u$ et $v$ variant dans un borné $B$ de $H^{s}\left(\mathbb{R}^{n}\right)$,

$$
r(u)-r(v)=\sum_{k=2}^{\infty}\left(u_{k}-v_{k}\right) r_{k}(u)+\sum_{k=2}^{\infty} v_{k}\left(r_{k}(u)-r_{k}(v)\right) .
$$

On applique alors le théorème 2.7 à chacune de ces deux séries. Il suffit pour cela d'établir les estimations

$$
\left\|\partial^{\alpha} r_{k}(u)\right\|_{0} \leq \varepsilon_{k} 2^{k(|\alpha|-s)} \quad \text { et }\left\|\partial^{\alpha}\left(r_{k}(u)-r_{k}(v)\right)\right\|_{0} \leq \delta_{k} 2^{k(|\alpha|-s)}
$$


pour $0 \leq|\alpha| \leq N$ avec $N$ assez grand $(N>s+\varrho$ suffit $)$ et des suites $\left(\varepsilon_{k}\right)$ et $\left(\delta_{k}\right)$ vérifiant

$$
\left\|\left(\varepsilon_{k}\right)\right\|_{l^{2}} \leq C_{B}\|u\|_{s} \text { et }\left\|\left(\delta_{k}\right)\right\|_{l^{2}} \leq C_{B}\|u-v\|_{s},
$$

la constante $C_{B}$ ne dépendant que du borné $B$ de $H^{s}\left(\mathbb{R}^{n}\right)$. L'estimation sur $\partial^{\alpha} r_{k}(u)$ découle du lemme suivant :

Lemme 2.11. Soit $G \in C^{\infty}(\mathbb{R})$ et $B$ une partie bornée de $H^{s}\left(\mathbb{R}^{n}, \mathbb{R}\right), s>n / 2$. Pour tout entier $N>s$, il existe une constante $C_{B}$ telle que, pour tout $u \in B$, on ait

$$
\left\|\partial^{\alpha}\left[G\left(S_{k}(u)\right)-S_{k-k_{0}}(G(u))\right]\right\|_{0} \leq \varepsilon_{k} 2^{k(|\alpha|-s)},
$$

pour $0 \leq|\alpha| \leq N$, où la suite $\left(\varepsilon_{k}\right)$ vérifie $\left\|\left(\varepsilon_{k}\right)\right\|_{l^{2}} \leq C_{B}\|u\|_{s}$, et l'entier $k_{0}$ est fixé.

On renvoie à l'article [8] où ce lemme (Proposition 1 de [8]) est démontré même si l'estimation de $\left\|\left(\varepsilon_{k}\right)\right\|_{l^{2}}$ par rapport à $B$ et $u$ n'y est pas. En fait, il suffit de suivre les détails de cette preuve pour voir que cette estimation est vraie.

Enfin, comme

$r_{k}(u)-r_{k}(v)=\int_{0}^{1}\left[F^{\prime}\left(S_{k-1}(u)+t u_{k}\right)-F^{\prime}\left(S_{k-1}(v)+t v_{k}\right)\right] d t-S_{k-3}\left(F^{\prime}(u)-F^{\prime}(v)\right)$,

l'estimation sur $\partial^{\alpha}\left(r_{k}(u)-r_{k}(v)\right)$ vient de l'analogue suivant du lemme précédent:

Lemme 2.12. Soit $G \in C^{\infty}(\mathbb{R})$ et $B$ une partie bornée de $H^{s}\left(\mathbb{R}^{n}, \mathbb{R}\right), s>n / 2$. Pour tout entier $N>s$, il existe une constante $C_{B}$ telle que, pour tous $u, v \in B$, on ait

$$
\left\|\partial^{\alpha}\left[G\left(S_{k}(u)\right)-G\left(S_{k}(v)\right)-S_{k-k_{0}}(G(u)-G(v))\right]\right\|_{0} \leq \delta_{k} 2^{k(|\alpha|-s)},
$$

pour $0 \leq|\alpha| \leq N$, où la suite $\left(\delta_{k}\right)$ vérifie $\left\|\left(\delta_{k}\right)\right\|_{l^{2}} \leq C_{B}\|u-v\|_{s}$, et l'entier $k_{0}$ est fixé.

Prueve : La méthode de preuve de ce lemme est la même que celle du lemme précédent (voir [8]). Cependant, on utilise en plus le caractère localement lipschitzien de $G$.

Le cas $m>1$ se traite par le même type d'arguments en écrivant

$$
\begin{aligned}
F\left(u_{1}, \ldots, u_{m}\right)= & F\left(S_{-1}\left(u_{1}, \ldots, u_{m}\right)\right) \\
& +\sum_{k=0}^{\infty} F\left(S_{k}\left(u_{1}, \ldots, u_{m}\right)\right)-F\left(S_{k-1}\left(u_{1}, \ldots, u_{m}\right)\right),
\end{aligned}
$$

où on a noté $S_{k}\left(u_{1}, \ldots, u_{m}\right)=\left(S_{k}\left(u_{1}\right), \ldots, S_{k}\left(u_{m}\right)\right)$.

Remarque : Dans cet article, nous appliquerons la formule de Bony à l'expression $F(u, \nabla u, \bar{u}, \nabla \bar{u})$, où $u \in H^{n / 2+1+\varrho}\left(\mathbb{R}^{n}\right)$ est à valeurs complexes. C'est possible car on peut écrire

$$
F(u, \nabla u, \bar{u}, \nabla \bar{u})=G(\mathscr{R}(u), \nabla \mathscr{R}(u), \mathscr{I}(u), \nabla \mathscr{I}(u))
$$


avec $G\left(x_{1}, x_{2}, y_{1}, y_{2}\right)=F\left(x_{1}+i y_{1}, x_{2}+i y_{2}, x_{1}-i y_{1}, x_{2}-i y_{2}\right)$ qui est une fonction $C^{\infty}$ de $\mathbb{R}^{2 n+2}$ dans $\mathbb{C}$. On applique alors la formule de Bony à $G$ et on obtient que

$$
F(u, \nabla u, \bar{u}, \nabla \bar{u})=T_{\partial_{x_{1}} G} \mathscr{R}(u)+T_{\partial_{x_{2}} G} \nabla \mathscr{R}(u)+T_{\partial_{y_{1}} G} \mathscr{I}(u)+T_{\partial_{y_{2}} G} \nabla \mathscr{I}(u)+r .
$$

En utilisant que

$$
\mathscr{R}(u)=\frac{u+\bar{u}}{2}, \quad \mathscr{I}(u)=\frac{u-\bar{u}}{2 i}, \quad \partial_{z}=\frac{1}{2}\left(\partial_{x}-i \partial_{y}\right) \quad \text { et } \quad \partial_{\bar{z}}=\frac{1}{2}\left(\partial_{x}+i \partial_{y}\right),
$$

puis la linéarité de $T_{b}$ par rapport à $b$, on obtient la formule utilisée dans cet article :

$$
F(u, \bar{u}, \nabla u, \nabla \bar{u})=T_{\partial_{u} F} u+T_{\partial_{\bar{u}} F} \bar{u}+T_{\partial_{\nabla u} F} \nabla u+T_{\partial_{\nabla \bar{u}} F} \nabla \bar{u}+r(u),
$$

avec $r(u) \in H^{n / 2+2 \varrho}\left(\mathbb{R}^{n}\right)$.

Variante paradifférentielle : Dans cet article, pour des raisons techniques, nous utiliserons une paratroncature $\chi(\xi, \eta)$ de la forme

$$
\chi_{1}\left(\xi /|\eta|^{\delta}\right)\left(1-\psi_{1}(\eta)\right),
$$

où $\psi_{1}, \chi_{1} \in C^{\infty}\left(\mathbb{R}^{n}\right), \psi_{1}$ vaut 1 au voisinage de 0 et est à support dans $B(0, h)$, et $\chi_{1}$ est à support dans $B(0, \varepsilon)$ et vaut 1 sur $B\left(0, \varepsilon^{\prime}\right)$, où $\varepsilon$ et $\varepsilon^{\prime}$ sont deux réels tels que $0<\varepsilon^{\prime}<\varepsilon<1$, ce qui, bien entendu, modifie la quantification paradifférentielle de Bony. L'opérateur associé, noté $T_{a}^{\delta}$, n'est pas un opérateur paradifférentiel classique puisque $T_{a}^{\delta}=\widetilde{a}^{\delta}(x, D)$ avec $\widetilde{a}^{\delta} \in S_{1, \delta}^{m}$, ce qui est une meilleure classe que $S_{1,1}^{m}$ si $\delta<1$. Cependant, avec cette définition, les restes ne seront plus $\varrho$-régularisants, mais seulement $\delta \varrho$-régularisants.

Plus généralement, étant donnée une fonction $b \in L^{\infty}\left(\mathbb{R}^{n}\right)$, on posera

$$
\widetilde{b}^{\delta}(x, \xi)=\left(1-\psi_{1}(\xi)\right)|\xi|^{\delta n} \int_{\mathbb{R}^{n}} \mathscr{F}^{-1}\left(\chi_{1}\right)\left(|\xi|^{\delta}(x-y)\right) b(y) d y,
$$

où $0 \leq \delta \leq 1$ et $\psi_{1}, \chi_{1}$ sont comme ci-dessus. On utilisera aussi la notation $T_{b}^{\delta}=$ $\tilde{b}^{\delta}(x, D)$ et on notera que $T_{b}^{1}$ n'est autre que l'opérateur paradifférentiel (de paramultiplication) $T_{b}$.

Nous aurons besoin du lemme technique suivant :

Lemme 2.13. Soit $\delta \in[0,1]$ et $\varrho \geq 0$. Si $b \in C^{\varrho}\left(\mathbb{R}^{n}\right)$ et $\operatorname{supp}(b) \subset Q_{\mu}=\mu+[0,1]^{n}$, alors $\widetilde{b}^{\delta} \in C^{\infty}$ et, pour tous $\alpha, \beta \in \mathbb{N}^{n}$, tout $N \in \mathbb{N}$, tout $\mu \in \mathbb{Z}^{n}$, on a

$$
\begin{aligned}
& \left\langle x-x_{\mu}\right\rangle^{N}\left|\partial_{\xi}^{\beta} \partial_{x}^{\alpha} \widetilde{b}^{\delta}(x, \xi)\right| \leq A_{\alpha, \beta, N}\|b\|_{C}\langle\xi\rangle^{\delta(|\alpha|-\varrho)-|\beta|} \quad \text { pour }|\alpha|>\varrho, \\
& \left\langle x-x_{\mu}\right\rangle^{N}\left|\partial_{\xi}^{\beta} \partial_{x}^{\alpha} \widetilde{b}^{\delta}(x, \xi)\right| \leq A_{\alpha, \beta, N}\|b\|_{C}\langle\xi\rangle^{-|\beta|} \text { pour }|\alpha| \leq \varrho,
\end{aligned}
$$

où $A_{\alpha, \beta, N}$ désigne une constante positive indépendante de $\mu$ et $\sigma$. On a donc $\tilde{b}^{\delta} \in S_{1, \delta}^{0}$ en particulier. Sans la condition de support sur b, la conclusion de ce lemme reste vraie avec $N=0$. 
Preuve : Pour simplifier, on écrit $\widetilde{b}$ au lieu de $\widetilde{b}^{\delta}$ et on omet le facteur $1-\psi_{1}(\xi)$ qui ne pose pas de problème. On rappelle que $1-\psi_{1}$ est nulle au voisinage de 0 , et plus précisément, on a $|\xi| \geq 1$ et donc $\langle\xi\rangle \leq \sqrt{2}|\xi|$. Pour tout multi-indice $\alpha$ et tout $\alpha^{\prime} \leq \alpha$ tel que $\left|\alpha-\alpha^{\prime}\right| \leq \varrho$, on a

$$
\left\langle x-x_{\mu}\right\rangle^{N} \partial_{x}^{\alpha} \widetilde{b}(x, \xi)=|\xi|^{\delta n} \int_{\mathbb{R}^{n}}|\xi|^{\delta\left|\alpha^{\prime}\right|}\left(\partial_{x}^{\alpha^{\prime}} \widehat{\chi}_{1}\right)\left(|\xi|^{\delta}(x-y)\right) \cdot\left\langle x-x_{\mu}\right\rangle^{N}\left(\partial_{x}^{\alpha-\alpha^{\prime}} b\right)(y) d y .
$$

Quand $\alpha=0$, on a, pour tout multi-indice $\beta$,

$$
\left\langle x-x_{\mu}\right\rangle^{N} \partial_{\xi}^{\beta} \widetilde{b}(x, \xi)=\sum_{\gamma \leq \beta}\left(\begin{array}{l}
\beta \\
\gamma
\end{array}\right) \partial_{\xi}^{\beta-\gamma}\left(|\xi|^{\delta n}\right) \int_{\mathbb{R}^{n}} \partial_{\xi}^{\gamma}\left(\widehat{\chi}_{1}\left(|\xi|^{\delta}(x-y)\right)\right) \cdot\left\langle x-x_{\mu}\right\rangle^{N} b(y) d y .
$$

Or, en vertu de la formule de Fàa di Bruno,

$$
\partial_{\xi}^{\gamma}\left(\widehat{\chi}_{1}\left(|\xi|^{\delta}(x-y)\right)\right)=\sum_{\substack{\nu \in \mathbb{N}^{n} \\|\nu|=q \leq|\gamma|}} \sum_{\substack{\gamma=\gamma_{1}+\cdots+\gamma_{q} \\ \gamma_{i} \neq 0}} \partial^{\nu} \widehat{\chi}_{1}\left(|\xi|^{\delta}(x-y)\right) \partial_{\xi}^{\gamma_{1}}\left(|\xi|^{\delta}\right) \ldots \partial_{\xi}^{\gamma_{q}}\left(|\xi|^{\delta}\right)(x-y)^{\nu}
$$

ce qui permet de majorer comme suit :

$$
\left|\partial_{\xi}^{\gamma}\left(\widehat{\chi}\left(|\xi|^{\delta}(x-y)\right)\right)\right| \leq \sum_{|\nu| \leq|\gamma|} C_{\nu}\left|\partial^{\nu} \widehat{\chi}_{1}\left(|\xi|^{\delta}(x-y)\right)\left(|\xi|^{\delta}(x-y)\right)^{\nu}\right||\xi|^{-|\gamma|} .
$$

De plus,

$$
\left\langle x-x_{\mu}\right\rangle^{N} \leq\langle x-y\rangle^{N}\left\langle y-x_{\mu}\right\rangle^{N} \leq C_{N}^{\prime}\langle x-y\rangle^{N} \leq C_{N}^{\prime \prime}\left(1+\max \left(|x-y|,|x-y|^{N}\right)\right)
$$

sur le support de $b$. Ces arguments permettent d'obtenir le résultat dans le cas $|\alpha|=0$ sachant que $\|b\|_{L^{\infty}} \leq\|b\|_{C} \varrho$. Dans le cas $|\alpha| \leq \varrho$, le raisonnement est identique avec $\partial^{\alpha} b$ au lieu de $b$, cad avec $\alpha^{\prime}=0$.

Cas $|\alpha|>\varrho$ et $\varrho=[\varrho]$ : On prend $\alpha^{\prime}$ tel que $\left|\alpha-\alpha^{\prime}\right|=\varrho$ et on reprend le raisonnement ci-dessus avec $\partial^{\alpha-\alpha^{\prime}} b$ au lieu de $b$, puisqu'alors $\left|\alpha^{\prime}\right|=|\alpha|-\varrho$.

Cas $|\alpha|>\varrho, \varrho$ non entier, et $\beta=0$ : On écrit $|\alpha|=\left|\alpha^{\prime}\right|+|\alpha|-\left|\alpha^{\prime}\right|$ avec $|\alpha|-\left|\alpha^{\prime}\right|=[\varrho]$ et $\left|\alpha^{\prime}\right| \geq 1$. Comme $\widehat{\chi}_{1} \in \mathscr{S}$, on a donc l'expression

$$
\partial_{x}^{\alpha} \widetilde{b}(x, \xi)=|\xi|^{\delta\left(n+\left|\alpha^{\prime}\right|\right)} \int_{\mathbb{R}^{n}}\left(\partial_{x}^{\alpha^{\prime}} \widehat{\chi}\right)\left(|\xi|^{\delta}(x-y)\right) \cdot\left(\partial^{\alpha-\alpha^{\prime}} b(y)-\partial^{\alpha-\alpha^{\prime}} b(x)\right) d y,
$$

et, si $\psi$ est une fonction dans $\mathscr{D}$ telle que $\psi\left(x-x_{\mu}\right)=1$ sur le support de $b$, on a

$$
\begin{aligned}
\langle x- & \left.x_{\mu}\right\rangle^{N}\left|\partial^{\alpha-\alpha^{\prime}} b(y)-\partial^{\alpha-\alpha^{\prime}} b(x)\right| \\
= & \left\langle x-x_{\mu}\right\rangle^{N}\left|\psi\left(y-x_{\mu}\right) \partial^{\alpha-\alpha^{\prime}} b(y)-\psi\left(x-x_{\mu}\right) \partial^{\alpha-\alpha^{\prime}} b(x)\right| \\
\leq & \left\langle x-x_{\mu}\right\rangle^{N}\left|\psi\left(x-x_{\mu}\right)\right|\left|\partial^{\alpha-\alpha^{\prime}} b(y)-\partial^{\alpha-\alpha^{\prime}} b(x)\right| \\
& +\left\langle x-x_{\mu}\right\rangle^{N}\left|\psi\left(y-x_{\mu}\right)-\psi\left(x-x_{\mu}\right)\right|\left|\partial^{\alpha-\alpha^{\prime}} b(y)\right| \\
\leq & \left(1+C_{N}\right)\left(|x-y|^{\varrho-[\varrho]}\|b\|_{C \varrho}+\langle x-y\rangle^{N}|x-y| \sup _{y}\left\langle y-x_{\mu}\right\rangle^{N}\left|\partial^{\alpha-\alpha^{\prime}} b(y)\right|\right) .
\end{aligned}
$$


Ceci permet de majorer $\left\langle x-x_{\mu}\right\rangle^{N}\left|\partial_{x}^{\alpha} \widetilde{b}(x, \xi)\right|$ et d'obtenir l'estimation

$$
\left\langle x-x_{\mu}\right\rangle^{N}\left|\partial_{x}^{\alpha} \tilde{b}(x, \xi)\right| \leq C_{N, \alpha}\|b\|_{C \varrho}|\xi|^{\delta(|\alpha|-\varrho)} .
$$

Enfin, le cas $\beta \neq 0$ s'obtient en combinant les raisonnements précédents.

Proposition 2.14. Si $b \in L^{\infty}\left(\mathbb{R}^{n}\right)$ et $\delta \in[0,1]$, l'opérateur $T_{b}^{\delta}$ est, pour tout $s \in \mathbb{R}$, borné sur $H^{s}\left(\mathbb{R}^{n}\right)$ et sa norme d'opérateur est majorée par une constante fois $\|b\|_{L^{\infty}}$.

Preuve : D'après le lemme précédent, $T_{b}^{\delta}=\widetilde{b}^{\delta}(x, D) \in O p\left(S_{1, \delta}^{0}\right)$, classe dont les opérateurs sont bornés sur $H^{s}\left(\mathbb{R}^{n}\right)$ quand $\delta<1$. Pour $\delta=1$, il s'agit de l'opérateur de paramultiplication de J.-M. Bony qui est donc borné sur $H^{s}\left(\mathbb{R}^{n}\right)$.

Lemme 2.15. Soit $R>0$. Si $b \in C^{\varrho}\left(\mathbb{R}^{n}\right)$ et $\widehat{b}=0$ dans $B(0, R)$, alors

$$
\|b\|_{L^{\infty}} \leq C_{n} R^{-\varrho}\|b\|_{C} \varrho .
$$

Proposition 2.16. Si $b \in C^{\varrho}\left(\mathbb{R}^{n}\right)$ et $\delta \in[0,1]$, alors, pour tout $s \in \mathbb{R}, T_{b}-T_{b}^{\delta}$ est continu de $H^{s}\left(\mathbb{R}^{n}\right)$ dans $H^{s+\delta \varrho}\left(\mathbb{R}^{n}\right)$ et sa norme d'opérateur est majorée par une constante fois $\|b\|_{C}$, pourvu qu'on utilise dans la définition de $T_{b}$ et $T_{b}^{\delta}$ les mêmes troncatures $\chi_{1}$ et $\psi_{1}$.

Preuve : Soit $\left(u_{j}\right),\left(b_{k}\right)$ les suites des termes dyadiques de $u, b$, respectivement. On peut écrire, au moins au sens des distributions, pour $u \in \mathscr{S}\left(\mathbb{R}^{n}\right)$,

$\mathscr{F}\left(\left(T_{b_{k}}-T_{b_{k}}^{\delta}\right) u_{j}\right)(\xi)=\int_{\mathbb{R}^{n}}\left[\chi_{1}\left(\frac{\xi-\eta}{|\eta|}\right)-\chi_{1}\left(\frac{\xi-\eta}{|\eta|^{\delta}}\right)\right] \widehat{b}_{k}(\xi-\eta) \widehat{u}_{j}(\eta)\left(1-\psi_{1}(\eta)\right) d \eta$.

Il découle des propriétés de support de $\chi_{1}, b_{k}$ et $u_{j}$ que, sur le support d'intégration, l'on a $2^{k} \sim|\xi-\eta| \geq$ Cste $|\eta|^{\delta} \sim$ Cste $2^{j \delta}$. Il existe donc un entier $k_{0}$ tel que l'on puisse écrire

$$
\left(T_{b}-T_{b}^{\delta}\right), u_{j}=\sum_{k \geq j \delta-k_{0}}\left(T_{b_{k}}-T_{b_{k}}^{\delta}\right) u_{j}
$$

On en déduit, par application de la proposition 2.14 et du lemme 2.15, l'estimation

$$
\left\|\left(T_{b}-T_{b}^{\delta}\right) u_{j}\right\|_{L^{2}} \leq A_{1} \sum_{k \geq j \delta-k_{0}} 2^{-k \varrho}\|b\|_{C} \varrho\left\|u_{j}\right\|_{L^{2}} \leq A_{2} 2^{-j \varrho \delta}\|b\|_{C} \varrho\left\|u_{j}\right\|_{L^{2}},
$$

ce qui donne le résultat sachant que les termes $\left(T_{b}-T_{b}^{\delta}\right) u_{j}$ sont à spectre inclus dans une couronne de la forme $c_{1} 2^{j} \leq|\xi| \leq c_{2} 2^{j}$, avec $0<c_{1}<c_{2}$, comme on peut le vérifier facilement.

Definition 2.17. Pour tout entier naturel $k, C^{k} S_{\gamma, \delta}^{m}$ est l'ensemble des fonctions de $C^{k}\left(\mathbb{R}^{2 n}\right)$ telle que $(\xi \mapsto a(x, \xi)) \in C^{\infty}\left(\mathbb{R}^{n}, C^{k}\left(\mathbb{R}^{n}\right)\right)$ et, pour tout $\alpha$ et $\beta$ des multi-indices de $\mathbb{N}^{n}$ avec $|\alpha| \leq k$,

$$
\left|\partial_{x}^{\alpha} \partial_{\xi}^{\beta} a(x, \xi)\right| \leq A_{\alpha, \beta}|\xi|^{-\gamma|\beta|+\delta|\alpha|+m} .
$$


Théorème 2.18 (Inégalité de Gårding précisée pour un système). Soit $C$ une matrice $l \times l$ dont les éléments sont dans $C^{k} S_{1,0}^{m}$ avec $k$ un entier supérieur où égal à 2. Supposons de plus que le symbole $c(x, \xi)$ (une $l \times l$ matrice) de $C$ vérifie

$$
\left\langle\left(c(x, \xi)+c^{*}(x, \xi)\right) \eta, \eta\right\rangle \geq 0
$$

pour tout $\eta \in \mathbb{C}^{l}$ et pour tout $|\xi| \geq A_{0}$, où $c^{*}$ désigne la matrice adjointe de $c$ et $\langle.,$.$\rangle le produit hermitien canonique de \mathbb{C}^{l}$. On a alors, pour tout $\vec{u} \in \mathscr{S}\left(\mathbb{R}^{n}, \mathbb{C}^{l}\right)$,

$$
\mathscr{R}\langle C \vec{u}, \vec{u}\rangle \geq-A\left\|J^{(m-1) / 2} \vec{u}\right\|_{0}^{2}
$$

où $A$ ne dépend seulement que de $n, l, A_{0}$ et des semi-normes de $c$.

Preuve : Voir [15].

Definition 2.19 (La classe de symboles $S_{\gamma, \delta}^{m, \varrho}$ avec $\gamma \geq \delta$ ). Dans le cas où $\gamma=1$, une fonction $s \in C^{\infty}\left(\mathbb{R}^{n} \times \mathbb{R}^{n}\right)$ appartient à la classe $S_{1, \delta}^{m, \varrho}$ si, pour tous multiindices $\alpha, \beta \in \mathbb{N}^{n}$, on a, pour $|\alpha| \leq \varrho$,

$$
\left|\partial_{x}^{\alpha} \partial_{\xi}^{\beta} s(x, \xi)\right| \leq A_{\alpha, \beta}(1+|\xi|)^{m-|\beta|},
$$

et, pour $|\alpha|>\varrho$,

$$
\left|\partial_{x}^{\alpha} \partial_{\xi}^{\beta} s(x, \xi)\right| \leq A_{\alpha, \beta}(1+|\xi|)^{m-|\beta|+\delta(|\alpha|-\varrho)} .
$$

Remarques: (i) Ces classes de symboles exotiques sont introduites pour pouvoir suivre précisément l'évolution de la régularité $\varrho$ et essayer ainsi de faire fonctionner les démonstrations avec un $\varrho$ le plus petit possible.

(ii) On remarque que $S_{\gamma, \delta}^{m, \varrho} \subset S_{\delta, \delta}^{m}$, pour $0 \leq \delta \leq 1$, on peut donc appliquer le théorème de Calderón-Vaillancourt aux opérateurs à symbole dans ces classes.

Lemme 2.20. Soit $\theta$ une fonction régularisante. On pose, $\forall m \in \mathbb{N}^{*}$,

$$
u_{m}=m^{n} \int_{\mathbb{R}^{n}} \theta(m(x-y)) u(y) d y .
$$

On a

$$
\begin{aligned}
& \forall M \in \mathbb{N}, \quad \forall m \in \mathbb{N}^{*},\left\|u_{m}\right\|_{C^{M}} \leq A_{n, M}\left(1+m^{M}\right)\|u\|_{L^{\infty}}, \\
& \forall \varrho \in \mathbb{R}^{*+}, \forall m \in \mathbb{N}^{*},\left\|u-u_{m}\right\|_{L^{\infty}} \leq \frac{\|u\|_{C}}{m^{\min (\varrho, 1)}} .
\end{aligned}
$$

Démonstration. Pour tout multi-indice $\alpha$ de longueur $M$, on a

$$
\partial_{x}^{\alpha} u_{m}(x)=m^{n+M} \int_{\mathbb{R}^{n}}\left(\partial_{x}^{\alpha} \theta\right)(m(x-y)) u(y) d y .
$$

donc, en posant $A=\sum_{\alpha \leq M} \int_{\mathbb{R}^{n}}\left|\left(\partial_{x}^{\alpha} \theta\right)(x)\right| d x$, on obtient l'inégalité (2.4). De plus, on a

$$
u(x)-u_{m}(x)=m^{n} \int_{\mathbb{R}^{n}} \theta(m(x-y))(u(y)-u(x)) d y
$$


or, si $\varrho \in] 0,1\left[\right.$, on a $|u(x)-u(y)| \leq A\|u\|_{C}|x-y|^{\varrho}$ donc

$$
\begin{aligned}
& \left|u(x)-u_{m}(x)\right| \leq A\|u\|_{C \varrho} m^{n} \int_{\mathbb{R}^{n}}|\theta(m(x-y))||x-y|^{\varrho} d y, \\
& \left|u(x)-u_{m}(x)\right| \leq A\|u\|_{C \varrho} m^{n-\varrho} \int_{\mathbb{R}^{n}}|\theta(m(x-y))| m^{\varrho}|x-y|^{\varrho} d y,
\end{aligned}
$$

or $\theta \in \mathscr{S}$, on obtient donc l'estimation (2.5), et si $\varrho \geq 1$ on a,

$$
|u(x)-u(y)| \leq A\|u\|_{C \varrho}|x-y|, \quad\left\|u-u_{m}\right\|_{L^{\infty}} \leq \frac{A\|u\|_{C}}{m} .
$$

\section{Schéma de démonstration}

Dans cette section sont donnés les principaux arguments utiles pour prouver le théorème 1.1. Cette preuve utilise de nombreux lemmes dont les démonstrations sont données dans la section 4 intitulée preuve des lemmes.

La constante $A$ étant le maximum des constantes obtenues dans les estimations des dérivées. Cette constante $A$.

Pour simplifier la rédaction, dans la suite, la constante $A$ utilisée qui ne dépend que de $n, s$ et $\varrho$, sera à chaque fois le maximum des constantes obtenues dans les estimations qui précèdent.

\subsection{Le cas paralinéaire}

Dans cette sous-section, nous traitons le problème de Cauchy pour l'"equation de Schrödinger généralisée linéaire définie ci-dessous.

On rappelle que $Q_{\mu}$ est le cube $\mu+[0,1]^{n}, \mu \in \mathbb{Z}^{n}$. On note $x_{\mu}$ le sommet du cube $Q_{\mu}$ image de 0 par la translation de vecteur $\mu$. La famille de cubes $\left\{Q_{\mu}\right\}_{\mu \in \mathbb{Z}^{n}}$ recouvre $\mathbb{R}^{n}$. On note $Q_{\mu}^{*}$ le cube de côté 2 obtenu par homothétie de centre le centre de $Q_{\mu}$. On note $c_{n}=\left\langle d_{n}\right\rangle$ où $d_{n}$ est la norme du vecteur $(1, \ldots, 1) \in \mathbb{R}^{n}$, longueur de la grande diagonale du cube $Q_{0}$ ainsi que des cubes $Q_{\mu}$ pour tout $\mu \in \mathbb{Z}^{n}$.

Théorème 3.1. Etant donné un nombre réel $s$ et un nombre réel $\delta \in[0,1[$, on considère le problème de Cauchy

$$
\left\{\begin{array}{l}
\partial_{t} u=i \mathscr{L} u+T_{b_{1}}^{\delta} \cdot \nabla_{x} u+T_{b_{2}}^{\delta} \cdot \nabla_{x} \bar{u}+C_{1} u+C_{2} \bar{u}+f(x, t) \\
u(x, 0)=u_{0} \in H^{s}\left(\mathbb{R}^{n}\right)
\end{array}\right.
$$

On suppose que $C_{1}$ et $C_{2}$ sont des opérateurs à symbole dans $S_{1, \delta_{1}}^{0}$ avec $0 \leq \delta_{1}<1$, et que $b_{1}, b_{2} \in C^{\varrho}\left(\mathbb{R}^{n}\right), \varrho>2$, et, plus précisément, que

$$
\left\{\begin{array}{l}
b_{k}(x)=\sum_{\mu \in \mathbb{Z}^{n}} \alpha_{k, \mu} \varphi_{k, \mu}(x), \quad k=1,2 \\
\operatorname{supp} \varphi_{k, \mu} \subseteq Q_{\mu}^{*}, \quad\left\|\varphi_{k, \mu}\right\|_{C} \leq 1, \quad \sum_{\mu}\left|\alpha_{k, \mu}\right| \leq A_{k} .
\end{array}\right.
$$


alors pour tout réel $s$, et tout $T>0$, l'équation (3.1) admet une unique solution dans $C\left([0, T] ; H^{s}\left(\mathbb{R}^{n}\right)\right.$ telle qu'il existe un réel $A$ tel que

$$
\begin{aligned}
& \sup _{0 \leq t \leq T}\|u(t)\|_{s}^{2} \leq A\left(\left\|u_{0}\right\|_{s}^{2}+I_{T}\left(J^{s} f, J^{s} u\right)\right), \\
& \left\|J^{s+1 / 2} u\right\|_{T}^{2} \leq A\left(\left\|u_{0}\right\|_{s}+I_{T}\left(J^{s} f, J^{s} u\right)\right)
\end{aligned}
$$

avec $\|\mid u\|_{T}=\sup _{\mu}\left(\int_{0}^{T}\left\|\left\langle x-x_{\mu}\right\rangle^{-2} u\right\|_{0}^{2} d t\right)^{1 / 2}$ et $I_{T}(f, u)$ est une somme de trois termes de la forme $\int_{0}^{T}|\langle G f, u\rangle| d t$ où $G \in O p S_{0,0}^{0}$ et $\|G\|_{\mathscr{L}\left(L^{2}\left(\mathbb{R}^{n}\right)\right)}$ ne dépendant que $n$ et de $s$.

Remarques : (i) La constante $A$ utilisée ci-dessus est de la forme $c_{1} \exp \left(c_{2} T\right)$ avec $c_{1}$ et $c_{2}$ deux constantes strictement positives.

(ii) Les estimations (3.2) et (3.3) impliquent les estimations (1.5), (1.6), (1.7) et (1.8).

(iii) Les coefficients $\alpha_{k, \mu}$ peuvent être supposés réels sans perte de généralité. En effet, s'ils sont complexes (non nuls), on utilise la forme trigonométrique, on incorpore la partie exponentielle aux $\varphi_{k, \mu}$. Le module cette partie exponentielle est de module 1 donc on a toujours $\left\|e^{i \arg \left(\alpha_{k, \mu}\right)} \varphi_{k, \mu}\right\|_{C \varrho} \leq 1$. Si $\alpha_{k, \mu} \in \mathbb{C}^{n}$, on applique ce raisonnement à chaque coordonnées.

(iv) Nous démontrons ce théorème sur l'intervalle $[0, T]$, le cas $[-T, 0]$ se traitant de façon analogue en étudiant l'équation vérifiée par $u(x,-t)$.

(v) Nous allons aussi travailler avec $s=0$ puisque le cas général peut être ramené à ce cas en appliquant $J^{s}$ à (3.1). Plus précisément, on pose $v=J^{s} u$ et donc $v_{0}=J^{s} u_{0} \in L^{2}$ pour $u_{0} \in H^{s}$. On obtient que $v$ est solution de

$$
\left\{\begin{array}{l}
\partial_{t} v=i \mathscr{L} v+T_{b_{1}}^{\delta} \cdot \nabla_{x} v+T_{b_{2}}^{\delta} \cdot \nabla_{x} \bar{v}+\widetilde{C}_{1} v+\widetilde{C}_{2} \bar{v}+\widetilde{f}(x, t) \\
v(x, 0)=v_{0} \in L^{2}\left(\mathbb{R}^{n}\right)
\end{array}\right.
$$

où $\widetilde{f}=J^{s} f$ et $\widetilde{C}_{k}=J^{s} C_{k} J^{-s}+\left[J^{s}, T_{b_{k}} . \nabla_{x}\right] J^{-s}, k=1$ ou 2 , qui sont des opérateurs dans $O p S_{1, \delta_{1}}^{0}$ car $C_{1}$ et $C_{2} \in O p S_{1, \delta_{1}}^{0}$ avec $0 \leq \delta_{1}<1$. Ce qui suffit pour obtenir le théorème 3.1 .

(vi) Dans la suite, $A$ désigne une constante polynômiale en $n, A_{1}, e^{A_{1}}$ et $A_{2}$.

Preuve du théorème 3.1. La démonstration de ce théorème est assez longue. Pour prouver ce théorème, on utilise les deux lemmes qui vont suivre, les lemmes 3.2 et 3.3 (effet régularisant). Pour prouver ces lemmes, on régularise les coefficients $b_{1}$ et $b_{2}$ de manière classique comme indiqué dans le lemme 2.20 , ce qui permet de travailler avec des opérateurs à symbole dans $S_{0,0}$ sans perte en régularité. Les restes obtenus s'estiment correctement car ils sont petits par passage à la limite. C'est le lemme 3.9 qui donne cette estimation sachant que pour prouver ce lemme on utilise l'estimation (2.5) donnée dans le lemme 2.20. On ne fait pas exactement un passage à la limite car sinon on ne peut obtenir l'existence un intervalle $\left[0, T_{0}\right]$ avec $T_{0}>0$ qui tend vers 0 si l'on passe à la limite et donc, avec la méthode utilisée, on ne peut étendre l'existence à tout intervalle $[0, T]$. 
Pour démontrer les lemmes 3.2 et 3.3 , on suppose donc que $u$ désigne une solution de (3.1) si elle existe avec les coefficients $b_{1}$ et $b_{2}$ vérifiant les hypothèses supplémentaires ci-dessous :

$$
b_{1}, b_{2} \in C^{\infty}\left(\mathbb{R}^{n}\right), \quad b_{k}(x)=\sum_{\mu \in \mathbb{Z}^{n}} \alpha_{k, \mu} \varphi_{k, \mu}(x), \quad k=1,2,
$$

avec

$$
\operatorname{supp} \varphi_{k, \mu} \subseteq Q_{\mu}^{*}, \quad\left\|\varphi_{k, \mu}\right\|_{C} \leq \leq 1, \quad \forall M \in \mathbb{N}, \varphi_{k, \mu} \in C^{M}\left(\mathbb{R}^{n}\right), \quad \sum_{\mu}\left|\alpha_{k, \mu}\right| \leq A_{k} .
$$

Lemme 3.2. Il existe un opérateur $\boldsymbol{C}$ inversible et borné dans $L^{2}\left(\mathbb{R}^{n}\right)$, un réel $A$ et deux entiers naturels $N$ et $M$ tels que, pour tout $T>0$,

$$
\begin{aligned}
\sup _{0 \leq t \leq T}\|\boldsymbol{C u}(t)\|_{0}^{2} \leq & \left\|\boldsymbol{C} u_{0}\right\|_{0}^{2}+2 \int_{0}^{T}|\langle\boldsymbol{C} f, \boldsymbol{C u}\rangle| d t \\
& +A \sup _{\mu, i}\left\|\varphi_{i, \mu}\right\|_{C^{M}}^{N}\left(R T \sup _{0 \leq t \leq T}\|u\|_{0}^{2}+\frac{1}{R}\left\|J^{1 / 2} u\right\| \|_{T}^{2}\right) .
\end{aligned}
$$

Lemme 3.3. Il existe un réel $A$ et un opérateur $\Psi \in O p S_{1,0}^{0}$ tels que, pour tout $T>0$, on $a$

$$
\left\|J^{1 / 2} u\right\|_{T}^{2} \leq A\left(\int_{0}^{T}|\langle\Psi f, u\rangle| d t+(1+T) \sup _{0 \leq t \leq T}\|u\|_{0}^{2}\right) .
$$

On a aussi, pour tout $R$ assez grand,

$$
\begin{aligned}
\left\|\mid J^{1 / 2} \boldsymbol{C u}\right\|_{T}^{2} \leq & A\left(\int_{0}^{T}|\langle\Psi \boldsymbol{C f}, \boldsymbol{C u}\rangle| d t+\sup _{0 \leq t \leq T}\|\boldsymbol{C u}\|_{0}^{2}\right) \\
& +\sup _{\mu, i}\left\|\varphi_{i, \mu}\right\|_{C^{M}}^{N}\left(\frac{A}{R}\|\| J^{1 / 2} u\|\|_{T}^{2}+A R T \sup _{0 \leq t \leq T}\|u(t)\|_{0}^{2}\right) .
\end{aligned}
$$

Remarques : (i) Les preuves des lemmes 3.2 et 3.3 suivent en partie les démonstrations faites dans [5] (Lemmes 3.2, 3.3).

(ii) La dépendance des coefficients par rapport à la norme $\sup _{\mu, i}\left\|\varphi_{i, \mu}\right\|_{C^{M}}$ n'est précisée que si la régularité $M$ utilisée est strictement supérieure à $\varrho$.

(iii) La preuve de l'effet régularisant (lemme 3.3) vérifié par $u$ ne coûte seulement que 2 crans de régularité pour les coefficeients $b_{1}$ et $b_{2}$. Ce qui coûte davantage en régularité pour obtenir l'effet régularisant vérifié par $\mathbf{C} u$ dans le cas où $\mathbf{C}$ est l'opérateur construit pour prouver le lemme 3.2, c'est l'utilisation de la continuité $L^{2}$ de $\mathbf{C}$.

(iv) À l'intérieur de ces preuves s'intercalent de nombreux lemmes ainsi que leur démonstration. Celle du lemme 3.2 nécessite notamment les lemmes 3.6 et 3.7, et, celle du lemme 3.3 utilise le lemme de Doi (voir [3]).

(v) Pour la preuve du lemme 3.3, on renvoie à la section 4.

Preuve du lemme 3.2 : Soit $\mathbf{C}$ un opérateur à symbole réel dans la classe $S_{0,0}^{0}$ tel que $\mathbf{C}$ soit borné dans $L^{2}$, et, inversible pour que des estimations de $\sup _{[0, T]}\|\mathbf{C} u\|_{0}^{2}$ en donnent pour $\sup _{[0, T]}\|u\|_{0}^{2}$. 
On a

$$
\partial_{t}\langle\mathbf{C} u, \mathbf{C} u\rangle=\left\langle\mathbf{C} \partial_{t} u, \mathbf{C} u\right\rangle+\left\langle\mathbf{C} u, \mathbf{C} \partial_{t} u\right\rangle,
$$

or

$$
\langle i \mathscr{L} \mathbf{C} u, \mathbf{C} u\rangle+\langle\mathbf{C} u, i \mathscr{L} \mathbf{C} u\rangle=0
$$

on obtient donc, comme dans [5], que

$$
\begin{aligned}
\frac{d}{d t}\langle\mathbf{C} u, \mathbf{C} u\rangle=2 \mathscr{R}\langle i[\mathbf{C}, \mathscr{L}] u, \mathbf{C} u\rangle+2 \mathscr{R}\left\langle\mathbf{C} T_{b_{1}}^{\delta} \cdot \nabla u, \mathbf{C} u\right\rangle \\
+2 \mathscr{R}\left\langle\mathbf{C} T_{b_{2}}^{\delta} \cdot \nabla \bar{u}, \mathbf{C} u\right\rangle+2 \mathscr{R}\langle\mathbf{C} u, \mathbf{C} f\rangle+2 \mathscr{R}\left(\left\langle\mathbf{C} C_{1} u, \mathbf{C} u\right\rangle+\left\langle\mathbf{C} C_{2} \bar{u}, \mathbf{C} u\right\rangle\right)
\end{aligned}
$$

On écrit alors que

$$
\begin{aligned}
2 \mathscr{R}\left\langle\mathbf{C} T_{b_{1}}^{\delta} \cdot \nabla u, \mathbf{C} u\right\rangle= & 2 \mathscr{R}\left\langle\mathbf{C} T_{\mathscr{R}\left(b_{1}\right)}^{\delta} \cdot \nabla u, \mathbf{C} u\right\rangle+2 \mathscr{R}\left\langle\mathbf{C} T_{i \mathscr{I}\left(b_{1}\right)}^{\delta} \cdot \nabla u, \mathbf{C} u\right\rangle \\
\frac{d}{d t}\langle\mathbf{C} u, \mathbf{C} u\rangle= & 2 \mathscr{R}\langle i[\mathbf{C}, \mathscr{L}] u, \mathbf{C} u\rangle+2 \mathscr{R}\left\langle\mathbf{C} T_{i \mathscr{I}\left(b_{1}\right)}^{\delta} \cdot \nabla u, \mathbf{C} u\right\rangle \\
& +2 \mathscr{R}\left\langle\mathbf{C} T_{\mathscr{R}\left(b_{1}\right)}^{\delta} \cdot \nabla u, \mathbf{C} u\right\rangle+2 \mathscr{R}\left\langle\mathbf{C} T_{b_{2}}^{\delta} \cdot \nabla \bar{u}, \mathbf{C} u\right\rangle \\
& +2 \mathscr{R}\langle\mathbf{C} u, \mathbf{C} f\rangle+2 \mathscr{R}\left(\left\langle\mathbf{C} C_{1} u, \mathbf{C} u\right\rangle+\left\langle\mathbf{C} C_{2} \bar{u}, \mathbf{C} u\right\rangle\right) .
\end{aligned}
$$

Par construction de $\mathbf{C}$, pour $M$ assez grand pour pouvoir appliquer le théorème de Calderón-Vaillancourt, pour tout $T>0$, on a

$$
\int_{0}^{T}\left|2 \mathscr{R}\left(\left\langle\mathbf{C} C_{1} u, \mathbf{C} u\right\rangle+\left\langle\mathbf{C} C_{2} \bar{u}, \mathbf{C} u\right\rangle\right)\right| d t \leq A\|\mathbf{C}\|_{\mathscr{L}\left(L^{2}\left(\mathbb{R}^{n}\right)\right)}^{2} \sup _{[0, T]}\|u\|_{0}^{2},
$$

En intégrant sur $\left[0, T_{0}\right]$, pour tout $T_{0} \in[0, T]$, on obtient

$$
\begin{aligned}
\left\|\mathbf{C} u\left(T_{0}\right)\right\|_{2}^{2} \leq & \left\|\mathbf{C} u_{0}\right\|_{0}^{2}+\left|2 \mathscr{R} \int_{0}^{T_{0}}\left\langle\mathbf{C} T_{b_{2}}^{\delta} \cdot \nabla \bar{u}, \mathbf{C} u\right\rangle d t\right|+\left|2 \mathscr{R} \int_{0}^{T_{0}}\left\langle\mathbf{C} T_{\mathscr{R}\left(b_{1}\right)}^{\delta} \cdot \nabla u, \mathbf{C} u\right\rangle d t\right| \\
& +\left|2 \mathscr{R} \int_{0}^{T_{0}}\langle\mathbf{C} u, \mathbf{C} f\rangle d t\right|+A T\|\mathbf{C}\|_{\mathscr{L}\left(L^{2}\left(\mathbb{R}^{n}\right)\right)}^{2} \sup _{[0, T]}\|u\|_{0}^{2} \\
& +\left|2 \mathscr{R} \int_{0}^{T_{0}}\langle i[\mathbf{C}, \mathscr{L}] u, \mathbf{C} u\rangle d t+2 \mathscr{R} \int_{0}^{T_{0}}\left\langle\mathbf{C} T_{i \mathscr{I}\left(b_{1}\right)}^{\delta} \cdot \nabla u, \mathbf{C} u\right\rangle d t\right| .
\end{aligned}
$$

Dans la suite, nous allons construire $\mathbf{C}$ pour rendre le terme

$$
\left|2 \mathscr{R} \int_{0}^{T}\langle i[\mathbf{C}, \mathscr{L}] u, \mathbf{C} u\rangle d t+2 \mathscr{R} \int_{0}^{T}\left\langle\mathbf{C} T_{i \mathscr{I}\left(b_{1}\right)}^{\delta} . \nabla u, \mathbf{C} u\right\rangle d t\right|
$$

suffisamment petit, dans un sens que l'on précisera ultérieurement. Sachant que le symbole $\mathbf{c}(x, \xi)$ de $\mathbf{C}$ est réel, on a

$$
\mathscr{R}\left(\left\langle\mathbf{C} T_{i \mathscr{I}\left(b_{1}\right)}^{\delta} . \nabla u, \mathbf{C} u\right\rangle\right)=\left\langle\left(-\mathbf{c}(x, \xi) \mathscr{I}\left(\widetilde{b}_{1}^{\delta}(x, \xi)\right) \cdot \xi\right)(x, D) u, \mathbf{C} u\right\rangle+\mathscr{R}\left(\left\langle E_{2, R} u, \mathbf{C} u\right\rangle\right.
$$

avec $E_{2, R}=\mathbf{C} T_{i \mathscr{I}\left(b_{1}\right)}^{\delta} . \nabla-\left(\mathbf{c}(x, \xi) i \mathscr{I}\left(\widetilde{b}_{1}^{\delta}(x, \xi)\right) . i \xi\right)(x, D)$.

Construction de $\mathbf{C}$ : On pose $\widetilde{\xi}=\left(-\xi_{1}, . .,-\xi_{k}, \xi_{k+1}, \ldots, \xi_{n}\right)$ et $\mathbf{c}(x, \xi)$ le symbole de $\mathbf{C}$. Nous allons construire $\mathbf{C}$ de symbole $\mathbf{c}(x, \xi) \in S_{0,0}^{0}$. 
On a

$$
i[\mathbf{C}, \mathscr{L}]=-2\left(\widetilde{\xi} \cdot \nabla_{x} \mathbf{c}(x, \xi)\right)(x, D)+E
$$

où $E=\mathscr{L} \mathbf{c}(x, D) \in O p S_{0,0}^{0}$ avec, pour $M$ assez grand,

$$
\|E u\|_{0} \leq A \sup _{\mu}\left\|\varphi_{1, \mu}\right\|_{C^{M}}\|u\|_{0} .
$$

Le but est de rendre

$$
-2 \widetilde{\xi} \cdot \nabla_{x} \mathbf{c}(x, \xi)-\mathbf{c}(x, \xi) \mathscr{I}\left(\widetilde{b}_{1}^{\delta}(x, \xi)\right) \cdot \xi
$$

aussi petit que possible. On pose $\mathbf{c}(x, \xi)=\mathbf{c}_{R}(x, \xi)$ avec $R>1$. On souhaite aussi que $\mathbf{C}$ soit inversible sur $L^{2}$. On pose

$$
\mathbf{c}_{R}(x, \xi)=\exp \left(\gamma_{R}(x, \xi)\right) \quad \text { et } \quad \gamma_{R}(x, \xi)=\sum_{\mu \in \mathbb{Z}^{n}} \alpha_{1, \mu} \gamma_{R, \mu}(x, \xi),
$$

avec $\sum_{\mu}\left|\alpha_{1, \mu}\right| \leq A_{1}$ où les $\alpha_{1, \mu}$ sont les coefficients donnés dans l'hypothèse du théorème 3.1 de décomposition de $b_{1}$ suivant les cubes $Q_{\mu}$. On a alors que

$$
\begin{aligned}
\nabla_{x} \mathbf{c}_{R}(x, \xi) & =\nabla_{x} \gamma_{R}(x, \xi) \exp \left(\gamma_{R}(x, \xi)\right) \\
& =\nabla_{x} \gamma_{R}(x, \xi) \mathbf{c}_{R}(x, \xi)-2 \widetilde{\xi} \cdot \nabla_{x} \mathbf{c}(x, \xi)-\mathbf{c}(x, \xi) \mathscr{I}\left(\widetilde{b}_{1}^{\delta}(x, \xi)\right) \cdot \xi \\
& =\mathbf{c}_{R}(x, \xi)\left(-2 \widetilde{\xi} \cdot \nabla_{x} \gamma_{R}(x, \xi)-\mathscr{I}\left(\widetilde{b}_{1}^{\delta}(x, \xi)\right) \cdot \xi\right) \\
& =\mathbf{c}_{R}(x, \xi) \sum_{\mu} \alpha_{1, \mu}\left(-2 \widetilde{\xi} \cdot \nabla_{x} \gamma_{R, \mu}(x, \xi)-\mathscr{I}\left(\widetilde{\varphi}_{1, \mu}^{\delta}(x, \xi)\right) \cdot \xi\right)
\end{aligned}
$$

Construisons alors $\gamma_{R, \mu}$. Considérons tout d'abord la fonction

$$
\eta_{\mu}(x, \xi)=\frac{1}{2} \int_{0}^{\infty} \mathscr{I}\left(\widetilde{\varphi}_{1, \mu}^{\delta}(x+s \widetilde{\xi}, \xi)\right) \cdot \xi d s=\frac{1}{2} \int_{0}^{\infty} \mathscr{I}\left(\widetilde{\varphi}_{1, \mu}^{\delta}\left(x+s \frac{\widetilde{\xi}}{|\xi|}, \xi\right)\right) \cdot \frac{\xi}{|\xi|} d s .
$$

Lemme 3.4. On rappelle que $x_{\mu}$ est un des coins de $Q_{\mu}$. On a $\eta_{\mu} \in C^{\infty}$ et, pour tous multi-indices $\alpha$ et $\beta$,

$$
\left|\partial_{\xi}^{\beta} \partial_{x}^{\alpha} \eta_{\mu}(x, \xi)\right| \leq A_{\alpha, \beta}\left\langle x-x_{\mu}\right\rangle^{|\beta|}\langle\xi\rangle^{-|\beta|}\left\|\varphi_{1, \mu}\right\|_{C^{|\alpha|+|\beta|}}
$$

où $A_{\alpha, \beta}$ est une constante qui ne dépend que de $n, \alpha$ et $\beta$. De plus, on a

$$
2 \widetilde{\xi} \cdot \nabla_{x} \eta_{\mu}(x, \xi)=-\mathscr{I}\left(\widetilde{\varphi}_{1, \mu}^{\delta}(x, \xi)\right) \cdot \xi .
$$

Preuve : Les premières inégalités s'obtiennent en utilisant la formule de dérivation des fonctions composées utilisée dans la preuve du lemme 2.13 appliquée à

$$
\eta_{\mu}(x, \xi)=\frac{\left(1-\psi_{1}(|\xi|)\right)|\xi|^{\delta n}}{2} \int_{0}^{\infty} \int_{\mathbb{R}^{n}} \widehat{\chi}_{1}\left(|\xi|^{\delta} y\right) \mathscr{I}\left(\varphi_{1, \mu}\left(x+s \frac{\widetilde{\xi}}{|\xi|}-y\right)\right) \cdot \frac{\xi}{|\xi|} d y d s
$$

et le support de $\varphi_{1, \mu}$ qui donne, après intervertion des intégrales en $s$ et $y$, en appelant $d_{n}$ la longueur d'une grande diagonale du cube $Q_{0}$, que l'on intègre pour $s \in\left[x_{\mu_{k}}-x_{k}+y_{k}-d_{n}, x_{\mu_{k}}-x_{k}+y_{k}+d_{n}\right]$ qui est un intervalle de mesure $2 d_{n}$. Pour donner une idée de la démonstration, on traite ci-dessous les cas $|\beta|=0$, $|\beta|=1$ et $|\alpha|$ quelconque. 
Dans le cas $|\beta|=0$, on a, après intervention des intégrales,

$\partial_{x}^{\alpha} \eta_{\mu}(x, \xi)=\frac{\left(1-\psi_{1}(|\xi|)\right)|\xi|^{\delta n}}{2} \int_{\mathbb{R}^{n}} \widehat{\chi}_{1}\left(|\xi|^{\delta} y\right) \int_{0}^{\infty} \mathscr{I}\left(\partial_{x}^{\alpha} \varphi_{1, \mu}\left(x+s \frac{\widetilde{\xi}}{|\xi|}-y\right)\right) \cdot \frac{\xi}{|\xi|} d s d y$

En utilisant le support de $\varphi_{1, \mu}$, sachant que $0 \leq 1-\psi_{1} \leq 1$, on obtient

$$
\left|\partial_{x}^{\alpha} \eta_{\mu}(x, \xi)\right| \leq d_{n}\left\|\varphi_{1, \mu}\right\|_{C^{|\alpha|} \mid}|\xi|^{\delta n} \int_{\mathbb{R}^{n}}\left|\widehat{\chi}_{1}\left(|\xi|^{\delta} y\right)\right| d y=d_{n}\left\|\varphi_{1, \mu}\right\|_{C^{|\alpha|} \mid}\left\|\widetilde{\chi}_{1}\right\|_{L^{1}}
$$

On rappelle que $\varphi_{1, \mu}(x) \in \mathbb{C}^{n}$ et on note $\varphi_{1, \mu, k}(x)$ ses coordonnées. Dans le cas $|\beta|=1$, on a, pour tout $j \in \llbracket 1, n \rrbracket$,

$$
\begin{aligned}
\partial_{\xi_{j}} \partial_{x}^{\alpha} \eta_{\mu}(x, \xi) & \frac{1}{2}\left(\psi_{1}^{\prime}(|\xi|) \frac{\xi_{j}}{|\xi|}|\xi|^{\delta n}+\left(1-\psi_{1}(|\xi|)\right) \delta n|\xi|^{\delta n-1} \frac{\xi_{j}}{|\xi|}\right) \\
& \cdot \int_{\mathbb{R}^{n}} \widehat{\chi}_{1}\left(|\xi|^{\delta} y\right) \int_{0}^{\infty} \mathscr{I}\left(\partial_{x}^{\alpha} \varphi_{1, \mu}\left(x+s \frac{\tilde{\xi}}{|\xi|}-y\right)\right) \cdot \frac{\xi}{|\xi|} d s d y \\
+ & \frac{1}{2}\left(\left(1-\psi_{1}(|\xi|)\right)|\xi|^{\delta n}\right) \\
& \cdot \int_{\mathbb{R}^{n}}\left(\nabla \widehat{\chi}_{1}\right)\left(|\xi|^{\delta} y\right) \cdot y \delta|\xi|^{\delta-1} \frac{\xi_{j}}{|\xi|} \int_{0}^{\infty} \mathscr{I}\left(\partial_{x}^{\alpha} \varphi_{1, \mu}\left(x+s \frac{\widetilde{\xi}}{|\xi|}-y\right)\right) \cdot \frac{\xi}{|\xi|} d s d y \\
+ & \frac{1}{2}\left(\left(1-\psi_{1}(|\xi|)\right)|\xi|^{\delta n}\right) \int_{\mathbb{R}^{n}} \widehat{\chi}_{1}\left(|\xi|^{\delta} y\right) \int_{0}^{\infty} \mathscr{I}\left(\partial_{x}^{\alpha} \varphi_{1, \mu, 1}\left(x+s \frac{\widetilde{\xi}}{|\xi|}-y\right)\right) \cdot \frac{1}{|\xi|} d s d y \\
+ & \frac{1}{2}\left(\left(1-\psi_{1}(|\xi|)\right)|\xi|^{\delta n}\right) \\
& \cdot \int_{\mathbb{R}^{n}} \widehat{\chi}_{1}\left(|\xi|^{\delta} y\right) \int_{0}^{\infty} \sum_{k} \mathscr{I}\left(\left(\nabla_{x_{k}} \partial_{x}^{\alpha} \varphi_{1, \mu, k}\right)\left(x+s \frac{\tilde{\xi}}{|\xi|}-y\right)\right) \cdot s \partial_{\xi_{j}}\left(\frac{\tilde{\xi}}{|\xi|}\right) \frac{\xi_{k}}{|\xi|} d s d y .
\end{aligned}
$$

Les trois premiers termes de la somme ci-dessus se traitent comme dans le cas $|\beta|=0$ avec une puissance $|\xi|^{-|\beta|}$ en facteur sachant que sur le support de $1-\psi_{1}$, $|\xi| \geq 1$. C'est le quatrième terme que fait apparaître un facteur $\left\langle x-x_{\mu}\right\rangle^{|\beta|}$, en effet

$$
\begin{aligned}
\int_{\left[x_{\mu_{k}}-x_{k}+y_{k}-d_{n}, x_{\mu_{k}}-x_{k}+y_{k}+d_{n}\right] \cap \mathbb{R}^{+}}|s| d s \\
\leq d_{n} \max _{k}\left(2\left|x_{\mu_{k}}-x_{k}+y_{k}\right|\right) \leq d_{n}\left(\left|x_{\mu}-x\right|+|y|\right) .
\end{aligned}
$$

La puissance de $|y|$ est absorbée par $\left|\widehat{\chi}_{1}\left(|\xi|^{\delta} y\right)\right|$ car $\widehat{\chi}_{1} \in \mathscr{S}$ et $|\xi| \geq 1$ donc $|\xi|^{\delta}$ aussi $\operatorname{car} \delta \geq 0$.

Prouvons ensuite l'égalité donnée dans ce lemme. On a

$$
2 \widetilde{\xi} \cdot \nabla_{x} \eta_{\mu}(x, \xi)=-\int_{0}^{+\infty} \sum_{k} \xi_{k} \widetilde{\xi} \cdot \nabla_{x}\left(\mathscr{I}\left(\widetilde{\varphi}_{1, \mu, k}^{\delta}(x+s \widetilde{\xi}, \xi)\right)\right) d s,
$$


or

$$
\begin{aligned}
\nabla_{x}\left(\widetilde{\varphi}_{1, \mu, k}^{\delta}(x+s \widetilde{\xi}, \xi)\right) & =\left(\nabla_{x} \widetilde{\varphi}_{1, \mu, k}^{\delta}\right)(x+s \tilde{\xi}, \xi), \\
\left(\nabla_{x} \widetilde{\varphi}_{1, \mu, k}^{\delta}\right)(x+s \widetilde{\xi}, \xi) \cdot \widetilde{\xi} & =\frac{d}{d s}\left(\widetilde{\varphi}_{1, \mu, k}^{\delta}(x+s \widetilde{\xi}, \xi)\right)
\end{aligned}
$$

et

$$
\left|\widetilde{\varphi}_{1, \mu, k}^{\delta}(x+s \widetilde{\xi}, \xi)\right| \leq \frac{A}{\left\langle x-x_{\mu}+s \widetilde{\xi}\right\rangle} \quad \text { car } \quad\left\langle x-x_{\mu}\right\rangle^{N}\left|\varphi_{1, \mu, k}^{\delta}(x)\right| \leq A_{N}\left\langle d_{n}\right\rangle^{N} .
$$

On obtient donc l'égalité donnée dans le lemme 3.4.

On définit alors

$$
v_{\mu}(x, \xi)=\frac{\eta_{\mu}(x, \xi)+\eta_{\mu}(x,-\xi)}{2} .
$$

La fonction $v_{\mu}$ est paire en $\xi$ et vérifie le lemme 3.4. Soit $\psi \in C_{0}^{\infty}(\mathbb{R})$ telle que $\psi(x)=0$ si $|x|>1$ et $\psi(x)=1$ si $|x| \leq 1 / 2$ et, $\theta_{1} \in C^{\infty}\left(\mathbb{R}^{n}\right)$ avec $\theta_{1}(\xi)=0$ si $|\xi| \leq 1$ et $\theta_{1}(\xi)=1$ si $|\xi| \geq 2$. Définissons ensuite

$$
\gamma_{R, \mu}(x, \xi)=\theta_{2}\left(\frac{\xi}{R}\right) \psi\left(R \frac{\left\langle x-x_{\mu}\right\rangle}{\langle\xi\rangle}\right) v_{\mu}(x, \xi),
$$

où $R$ est un réel dans $[1,+\infty[$, fixé très grand.

Remarque : On rappelle que $A$ est une constante polynômiale en $n, s, A_{1}, A_{2}$ et $e^{A_{1}}$, que $M$ est un entier naturel fixé assez grand ne dépendant que de $n$ et, que $R$ est un réel dans $[1,+\infty$ [ fixé très grand.

Lemme 3.5. (a) Le symbole $\gamma_{R, \mu}(x, \xi)$ est pair en $\xi$ et réel.

(b) On a

$$
\forall \alpha, \beta \in \mathbb{N}^{n}, \quad\left|\partial_{x}^{\alpha} \partial_{\xi}^{\beta} \gamma_{R, \mu}(x, \xi)\right| \leq A_{\alpha, \beta} \sup _{\mu}\left\|\varphi_{1, \mu}\right\|_{C|\alpha|+|\beta|} .
$$

Ici, comme dans la suite, une constante notée $A_{\alpha, \beta}$ est une constante qui ne dépend que de $n$, de $\alpha$ et $\beta$.

(c) On a

$$
-2 \widetilde{\xi} \cdot \nabla_{x} \gamma_{R, \mu}(x, \xi)-\mathscr{I}\left(\widetilde{\varphi}_{1, \mu}^{\delta}(x, \xi)\right) \cdot \xi=e_{R, \mu}(x, \xi)
$$

où

$$
e_{R, \mu}(x, D) \in O p S_{0,0}^{0} \quad \text { avec } \quad\left\|e_{R, \mu}(x, D)\right\|_{\mathscr{L}\left(L^{2}\left(\mathbb{R}^{n}\right)\right)} \leq A R\left\|\varphi_{1, \mu}\right\|_{C^{M}} .
$$

(d) On $a$

$\forall \alpha, \beta \in \mathbb{N}^{n}, \quad\left|\partial_{x}^{\alpha} \partial_{\xi}^{\beta} \partial_{\xi_{k}} \gamma_{R, \mu}(x, \xi)\right| \leq \frac{A_{\alpha, \beta}}{R} \sup _{\mu}\left\|\varphi_{1, \mu}\right\|_{C^{|\alpha|+|\beta|+1}}, \quad 1 \leq k \leq n$.

Pour la preuve de ce lemme 3.5, on renvoie à la section 4 .

Définissons maintenant $\sigma(\mathbf{C})$, le symbole de $\mathbf{C}$. On pose

$$
\sigma(\mathbf{C})=\mathbf{c}(x, \xi)=\mathbf{c}_{R}(x, \xi)=\exp \left(\gamma_{R}(x, \xi)\right) \quad \text { avec } \quad \gamma_{R}(x, \xi)=\sum_{\mu} \alpha_{1, \mu} \gamma_{R, \mu}(x, \xi)
$$


où les $\alpha_{1, \mu}$ désignent les coefficients de la décomposition de $b_{1}$ suivant les cubes $Q_{\mu}$ (hypothèse (S.2), théorème 3.1).

Lemme 3.6. (i) Le symbole $\boldsymbol{c}_{R}(x, \xi)$ est pair en $\xi$ et réel.

(ii) On $a$

$$
\forall \alpha, \beta \in \mathbb{N}^{n}, \quad\left|\partial_{x}^{\alpha} \partial_{\xi}^{\beta} \boldsymbol{c}(x, \xi)\right| \leq A_{\alpha, \beta} \sup _{\mu}\left\|\varphi_{1, \mu}\right\|_{C^{|\alpha|+|\beta|}} .
$$

(iii) On a

$$
\forall \alpha, \beta \in \mathbb{N}^{n}, \quad\left|\partial_{x}^{\alpha} \partial_{\xi}^{\beta} \partial_{\xi} \boldsymbol{c}(x, \xi)\right| \leq \frac{A_{\alpha, \beta}}{R} \sup _{\mu}\left\|\varphi_{1, \mu}\right\|_{C|\alpha|+|\beta|+1} .
$$

(iv) Pour $i=1$ ou $i=2$, tout $\varrho>1$, tout $T>0$ et tout $R \geq 1$, on a

$$
\int_{0}^{T}\left|\left\langle\left[C, T_{b_{i}}^{\delta} \cdot \nabla\right] u, C u\right\rangle\right| d t \leq A \sup _{\mu, i}\left\|\varphi_{i, \mu}\right\|_{C^{M}}^{3}\left(R T \sup _{[0, T]}\|u\|_{0}^{2}+\frac{1}{R}\left\|\mid J^{1 / 2} u\right\| \|_{T}^{2}\right) .
$$

(v) On a

$$
i[\boldsymbol{C}, \mathscr{L}]+C T_{i \mathscr{I}\left(b_{1}\right)}^{\delta} . \nabla_{x}=E_{1, R}+E_{2, R}+E
$$

où $E_{1, R} \in O p S_{0,0}^{0}$ est borné dans $L^{2}\left(\mathbb{R}^{n}\right)$ avec $\left\|E_{1, R}\right\|_{\mathscr{L}\left(L^{2}\right)} \leq A R \sup _{\mu}\left\|\varphi_{1, \mu}\right\|_{C^{M}}$ et $E \in O p S_{0,0}^{0}$ borné dans $L^{2}\left(\mathbb{R}^{n}\right)$ avec $\|E\|_{\mathscr{L}\left(L^{2}\right)} \leq A \sup _{\mu}\left\|\varphi_{1, \mu}\right\|_{C^{M}}$.

De plus, pour $S=C$ ou $S=\mathrm{Id}$, pour tout $T_{0} \in[0, T]$, on a

$$
\left|\int_{0}^{T_{0}}\left\langle E_{2, R} u, S u\right\rangle d t\right| \leq A \sup _{\mu}\left\|\varphi_{1, \mu}\right\|_{C^{M}}^{2}\left(R T \sup _{0 \leq t \leq T}\|u\|_{0}^{2}+\frac{1}{R}\left\|J^{1 / 2} u\right\| \|_{T}^{2}\right),
$$

(vi) On a

$$
\|u\|_{0} \leq A \sup _{\mu}\left\|\varphi_{1, \mu}\right\|_{C^{M}}\|\boldsymbol{C u}\|_{0}+\frac{A \sup _{\mu}\left\|\varphi_{1, \mu}\right\|_{C^{M}}^{2}}{R}\|u\|_{0} .
$$

Preuve : voir section 4.

Revenons à la preuve du lemme 3.2 et estimons $2 \mathscr{R} \int_{0}^{T}\left\langle\mathbf{C} T_{b_{2}}^{\delta} \nabla \bar{u}, \mathbf{C} u\right\rangle d t$, il suffit pour cela d'estimer $\left|\int_{0}^{T}\left\langle\mathbf{C} T_{b_{2}}^{\delta} \nabla \bar{u}, \mathbf{C} u\right\rangle d t\right|$.

Lemme 3.7. Soit $\boldsymbol{C}$ comme dans le lemme 3.6. On a

$$
\left|\int_{0}^{T}\left\langle C T_{b_{2}}^{\delta} \nabla \bar{u}, C u\right\rangle d t\right| \leq A \sup _{\mu, i}\left\|\varphi_{i, \mu}\right\|_{C^{M}}^{3}\left(R T \sup _{0 \leq t \leq T}\|u\|_{0}^{2}+\frac{1}{R} \mid\left\|J^{1 / 2} u\right\|_{T}\right) .
$$

Lemme 3.8. Soit $\boldsymbol{C}$ comme dans le lemme 3.6. On a

$$
\left|\int_{0}^{T}\left\langle C T_{\mathscr{R}\left(b_{1}\right)}^{\delta} \nabla u, C u\right\rangle d t\right| \leq A \sup _{\mu, i}\left\|\varphi_{i, \mu}\right\|_{C^{M}}^{3}\left(R T \sup _{0 \leq t \leq T}\|u\|_{0}^{2}+\frac{1}{R} \mid\left\|J^{1 / 2} u\right\|_{T}\right) .
$$

Preuve : voir section 4. 
En appliquant ces deux derniers lemmes, ainsi que la proposition (v) du lemme 3.6 à l'équation (3.7), on obtient l'inégalité du lemme 3.2.

Preuve du théorème 3.1 : On commence par démonstrer les estimations (3.2) et (3.3). On suppose donc que l'équation (3.1) admet une unique solution $u$. On régularise alors les coefficients $b_{i}$ en posant

$$
\varphi_{i, \mu, m}(x)=m^{n} \int_{\mathbb{R}^{n}} \theta(m(x-y)) \varphi_{i, \mu}(y) d y \quad \text { et } \quad b_{i, m}(x)=\sum_{\mu} \alpha_{i, \mu} \varphi_{i, \mu, m}(x) .
$$

où $\theta$ est une fonction régularisante définit comme dans le lemme 2.20. On rappelle que, par hypothèse, $\left\|\varphi_{1, \mu}\right\|_{C} \varrho \leq 1$. De plus, on remarque que, pour tout entier $M_{0}$,

$$
\begin{aligned}
\sup _{\mu, i}\left\|\left\langle x-x_{\mu}\right\rangle{ }^{M_{0}} \varphi_{i, \mu, m}\right\|_{C^{M}}^{N} & \leq A m^{M N} \sup _{\mu, i}\left\|\varphi_{i, \mu}\right\|_{L^{\infty}}^{N} \\
& \leq A m^{M N} \sup _{\mu, i}\left\|\varphi_{i, \mu}\right\|_{C^{e}}^{N} \leq A m^{N M}
\end{aligned}
$$

On obtient alors l'équation suivante

$$
\begin{aligned}
\partial_{t} u= & i \mathscr{L} u+T_{b_{1, m}}^{\delta} \cdot \nabla_{x} u+T_{b_{2, m}}^{\delta} \cdot \nabla_{x} \bar{u}+C_{1} u+C_{2} \bar{u} \\
& +\left(T_{b_{1}}-T_{b_{1, m}}\right) \cdot \nabla u+\left(T_{b_{2}}-T_{b_{2, m}}\right) \cdot \nabla \bar{u}+f(x, t)
\end{aligned}
$$

avec $b_{1, m}$ et $b_{2, m}$ qui vérifient l'hypothèse utilisée dans la preuve du lemme 3.2, la propriété de support pouvant être remplacée par l'estimation remarquée ci-dessus. On a notamment que

$$
b_{k, m}=\sum_{\mu} \alpha_{k, \mu} \varphi_{k, \mu, m}, \quad 1 \leq k \leq n .
$$

De plus, $u$ est solution de l'équation (3.10). On utilise alors le lemme 3.2 appliqué aux coefficients régularisés $b_{1, m}$ et $b_{2, m}$, avec $f$ remplacée par

$$
f+\sum_{\mu} \alpha_{1, \mu} f_{1, \mu}+\sum_{\mu} \alpha_{2, \mu} f_{2, \mu}
$$

où

$$
f_{1, \mu}=\left(T_{\varphi_{1, \mu}}^{\delta}-T_{\varphi_{1, \mu, m}}^{\delta}\right) \cdot \nabla_{x} u \quad \text { et } \quad f_{2, \mu}=\left(T_{\varphi_{2, \mu}}^{\delta}-T_{\varphi_{2, \mu, m}}^{\delta}\right) \cdot \nabla_{x} \bar{u} .
$$

Les estimations sont alors vraies pour un opérateur $\mathbf{C}$ dépendant de $m$ noté dans la suite $\mathbf{C}_{m}$. De plus, on a le lemme suivant.

Lemme 3.9. Pour tout $i \in\{1,2\}$, tout $\varrho \geq 2$, tout $m \geq 1$ et tout $m^{\prime} \geq m$, il existe une constante $A$ ne dépendant que de $n$, de $\varrho$ et de $\sup _{\mu}\left\|\varphi_{i, \mu}\right\|_{C e}$ telle que

$$
\begin{aligned}
& \left|\int_{0}^{T}\left\langle\boldsymbol{C}_{m}\left(T_{\varphi_{i, \mu}}-T_{\varphi_{i, \mu, m}}\right) \cdot \nabla \widetilde{u}, \boldsymbol{C}_{m} u\right\rangle d t\right| \\
& \leq\left.\frac{A}{m}\|\| J^{1 / 2} \boldsymbol{C}_{m} u\left\|_{T}^{2}+\left(\frac{A m^{\prime N M}}{R}+\frac{A m^{N M}}{m^{\prime}}\right)\right\|\left\|J^{1 / 2} u\right\|\right|_{T} ^{2}+A R m^{N M} T \sup _{[0, T]}\|u\|_{0}^{2},
\end{aligned}
$$

avec $\widetilde{u}=u$ ou $\widetilde{u}=\bar{u}$.

Preuve: Voir section 4. 
Revenons à la preuve du théorème 3.1. En utilisant le lemme 3.2, le lemme 3.9, on obtient que

$$
\begin{aligned}
\sup _{[0, T]}\left\|\mathbf{C}_{m} u\right\|_{0}^{2} \leq & A m^{N M}\left(\left\|u_{0}\right\|_{0}^{2}+T R \sup _{[0, T]}\|u\|_{0}^{2}+\int_{0}^{T}\left|\left\langle\mathbf{C}_{m} f, \mathbf{C}_{m} u\right\rangle\right| d t\right) \\
& +\frac{A}{m}\left\|\left|J^{1 / 2} \mathbf{C}_{m} u\|\|_{T}^{2}+\left(\frac{A m^{\prime N M}}{R}+\frac{A m^{N M}}{m^{\prime}}\right)\left\|\left|J^{1 / 2} u \|\right|_{T}^{2} .\right.\right.\right.
\end{aligned}
$$

On applique ensuite le lemme 3.3. On obtient alors

$$
\begin{aligned}
\sup _{[0, T]}\left\|\mathbf{C}_{m} u\right\|_{0}^{2} \leq & A m^{N M}\left(\left\|u_{0}\right\|_{0}^{2}+T R \sup _{[0, T]}\|u\|_{0}^{2}+\int_{0}^{T}\left|\left\langle\mathbf{C}_{m} f, \mathbf{C}_{m} u\right\rangle\right| d t\right) \\
& +\frac{A}{m}\left(\int_{0}^{T}\left|\left\langle\Psi \mathbf{C}_{m} f, \mathbf{C}_{m} u\right\rangle\right| d t+\sup _{0 \leq t \leq T}\left\|\mathbf{C}_{m} u\right\|_{0}^{2}\right) \\
& +\left(\frac{A m^{\prime N M}}{R}+\frac{A m^{N M}}{m^{\prime}}\right)\left(\int_{0}^{T}|\langle\Psi f, u\rangle| d t+\sup _{0 \leq t \leq T}\|u(t)\|_{0}^{2}\right) .
\end{aligned}
$$

Ce qui donne, pour $m \geq 2 A$ par exemple, une estimation de $\sup _{[0, T]}\left\|\mathbf{C}_{m} u\right\|_{0}^{2}$. On utilise que

$$
\|u\|_{0}^{2} \leq A m^{2 M}\left\|\mathbf{C}_{m} u\right\|_{0}^{2}+\frac{A m^{2 M}}{R}\|u\|_{0}^{2} .
$$

Ce qui permet d'obtenir les estimations (3.2) et (3.3) du théorème 3.1 pour $m^{\prime}$ assez grand devant $m, R \geq R_{m^{\prime}}$ assez grand et $T_{0}=T(R, m)$ assez petit avec

$$
I_{T_{0}}(f, u)=\int_{0}^{T_{0}}\left|\left\langle\mathbf{C}_{m}^{*} \Psi \mathbf{C}_{m} f, u\right\rangle\right| d t+\int_{0}^{T_{0}}\left|\left\langle\mathbf{C}_{m}^{*} \mathbf{C}_{m} f, u\right\rangle\right| d t+\int_{0}^{T_{0}}|\langle\Psi f, u\rangle| d t .
$$

Les estimations s'obtiennent ensuite pour tout $T>0$ en remarquant que

$$
v(x, t)=u\left(x, t+T_{0}\right),
$$

si elle existe, est solution d'une équation du type (3.14) avec $g(x, t)=f(x, t+$ $T_{0}$ ) au lieu de $f(x, t)$ donc on obtient que $v$ vérifie (3.2) et (3.3) sur le même intervalle $\left[0, T_{0}\right]$, les constantes intervenant dans la démonstration ne dépendant pas de $f$. En remarquant que

$$
I_{T_{0}}(f, u)+I_{T_{0}}(g, v)=I_{2 T_{0}}(f, u)
$$

et que $v_{0}=u\left(T_{0}\right)$ verifie (3.2) et (3.3), on obtient (3.2) et (3.3) sur l'intervalle $\left[0,2 T_{0}\right]$ avec une consante polynomiale en $A$, que l'on note aussi $A$ pour simplifier. En répétant l'opération, on obtient (3.2) et (3.3) sur tout intervalle $[0, T]$ avec une constante de la forme $A^{T / T_{1}}$ où $T_{1} \leq T_{0}$ tel qu'il exite un entier naturel $N$ tel que $N T_{1}=T$. Cette nouvelle constante $A^{T / T_{1}}$ est aussi notée $A$ dans la suite pour simplifier car, dans (3.2) et (3.3), $A$ peut dépendre de $T$. On remarque $A$ tend vers l'infini si $T$ tend vers l'infini et que $A$ est bornée si $T$ est dans un borné de $\mathbb{R}^{+}$. 
Pour obtenir l'unicité, on utilise que

$$
I_{T}(f, u) \leq A T \sup _{[0, T]}\|f\|_{0}^{2}+A T \sup _{[0, T]}\|u\|_{0}^{2} .
$$

On obtient, pour $T=T_{2}$ encore un peu plus petit,

$$
\sup _{\left[0, T_{2}\right]}\|u\|_{0} \leq A\left(\left\|u_{0}\right\|_{0}+\int_{0}^{T_{2}}\|f\|_{0} d t\right)
$$

L'unicité sur $\left[0, T_{2}\right]$ découle de cette dernière estimation. Pour obtenir l'unicité sur $[0, T]$, pour tout $T>0$, on remarque que, comme pour (3.2) et (3.3), cette dernière estimation est vérifiée pour tout $T>0$.

Existence. Soit $\varphi \in C^{\infty}\left(\mathbb{R}^{n}\right)$ une fonction plateau telle que $\varphi(\xi)=1$ si $|\xi| \leq 1 / 2$ et $\varphi(\xi)=0$ si $|\xi| \geq 1$. Pour tout $\varepsilon>0$, considérons l'équation suivante :

$$
\left\{\begin{array}{l}
\partial_{t} u=i \mathscr{L} u+T_{b_{1}}^{\delta} \cdot \nabla_{x} \varphi(\varepsilon D) u+T_{b_{2}}^{\delta} \cdot \varphi(\varepsilon D) \nabla_{x} \bar{u}+C_{1} u+C_{2} \bar{u}+f(x, t) \\
u(x, 0)=u_{0} \in H^{s}\left(\mathbb{R}^{n}\right)
\end{array}\right.
$$

Soit $T>0$ tel que $\sup _{[0, T]}\|f\|_{s}<+\infty$.

Cette équation admet une unique solution dans $C\left(\left[0, T_{\varepsilon}\right] ; H^{s}\left(\mathbb{R}^{n}\right)\right)$ avec $T_{\varepsilon} \in$ ] $0, T]$. On note cette solution $u_{\varepsilon}$.

En effet, la fonctionnelle $\Upsilon$ définie par

$$
\begin{aligned}
\Upsilon u=e^{i \mathscr{L} t} u_{0}+\int_{0}^{t} e^{i \mathscr{L}\left(t-t^{\prime}\right)}( & \left(T_{b_{1}}^{\delta} \cdot \nabla_{x} \varphi(\varepsilon D)+C_{1}\right) u \\
& \left.+\left(T_{b_{2}}^{\delta} \cdot \varphi(\varepsilon D) \nabla_{x}+C_{2}\right) \bar{u}+f\left(x, t^{\prime}\right)\right) d t^{\prime}
\end{aligned}
$$

admet un unique point fixe pour $T_{\varepsilon}$ assez petit.

En étudiant de la même manière le problème (3.11) mais avec la donnée initiale $u(x, 0)=u_{\varepsilon}\left(T_{\varepsilon}\right)$, on obtient l'existence d'une solution dans $C\left(\left[0,2 T_{\varepsilon}\right] ; H^{s}\left(\mathbb{R}^{n}\right)\right)$.

En réitérant ce raisonnement, on obtient que pour tout $T>0$, le problème (3.11) admet une unique solution dans $C\left([0, T] ; H^{s}\left(\mathbb{R}^{n}\right)\right)$.

La solution $u_{\varepsilon}$ de (3.11) vérifie les estimations d'énergie données dans le théorème 3.1 , uniformément en $\varepsilon$ car $u_{\varepsilon}$ vérifie les lemmes 3.3 et 3.2 uniformément en $\varepsilon$.

Ce résultat s'obtient en posant dans la preuve du lemme 3.3

$$
\gamma(x, \xi)=p\left(x-x_{\mu_{0}}, \xi\right)+\sum_{\mu}\left(\left|\alpha_{1, \mu}\right|+\left|\alpha_{2, \mu}\right|\right) p\left(x-x_{\mu}, \xi\right)
$$

et, dans la preuve du lemme 3.2, en remplaçant $\eta_{\mu}$ par $\widetilde{\eta}_{\mu}(x, \xi)=\varphi(\varepsilon \xi) \eta_{\mu}(x, \xi)$.

Sachant que $v=u_{\varepsilon}-u_{\varepsilon^{\prime}}$ est solution de l'équation (3.11) avec

$$
\tilde{f}=T_{b_{1}}^{\delta} \cdot \nabla_{x}\left(\varphi(\varepsilon D)-\varphi\left(\varepsilon^{\prime} D\right)\right) u_{\varepsilon}+T_{b_{2}}^{\delta} \cdot \nabla_{x}\left(\varphi(\varepsilon D)-\varphi\left(\varepsilon^{\prime} D\right)\right) \bar{u}_{\varepsilon}
$$


et $v_{0}=0$ donc $v$ vérifie les estimations d'energie du théorème 3.1. En utilisant ces mêmes estimations appliquées à $u_{\varepsilon}$ dans $\widetilde{f}$, pour tout $\varepsilon$, tout $\varepsilon^{\prime}$, tout $s \geq 2$, tout $u_{0}, f \in H^{s}$ et tout $T>0$, on a

$$
\int_{0}^{T}\|\widetilde{f}\|_{0} d t \leq\left(\varepsilon-\varepsilon^{\prime}\right) T A\left(\left\|u_{0}\right\|_{2}+\sup _{[0, T]}\|f\|_{2}\right) .
$$

On obtient que pour tout $T>0,\left(u_{\varepsilon}\right)$ est de Cauchy dans l'espace complet $C\left([0, T] ; L^{2}\left(\mathbb{R}^{n}\right)\right)$ et donc converge vers l'unique solution $u$ de (3.1) pour tout $u_{0} \in H^{2}$.

De plus, par un argument classique de densité de $S\left(\mathbb{R}^{n}\right) \subset H^{2}\left(\mathbb{R}^{n}\right)$ dans $L^{2}\left(\mathbb{R}^{n}\right)$, on peut approcher $u_{0}$ et $f$ dans $L^{2}$ par deux suites de fonctions $\left(u_{0, k}\right)$ et $\left(f_{k}\right)$ dans $S\left(\mathbb{R}^{n}\right)$. Soit $u_{k}$ l'unique solution de (3.1) (avec $f_{k}$ au lieu de $f$ ) dans $C\left([0, T] ; L^{2}\left(\mathbb{R}^{n}\right)\right)$ associée à $u_{0, k}$. D'après les estimations d'énergie données dans le théorème 3.1 , on a

$$
\sup _{[0, T]}\left\|u_{k}-u_{k^{\prime}}\right\|_{s} \leq A\left(\left\|u_{0, k}-u_{0, k}\right\|_{s}+T \sup _{[0, T]}\left\|f_{k}-f_{k^{\prime}}\right\|_{s}\right) .
$$

Sachant que $\left(u_{0, k}\right)$ et $\left(f_{k}\right)$ convergent alors on obtient que $\left(u_{k}\right)$ est de Cauchy dans l'espace $C\left([0, T] ; L^{2}\left(\mathbb{R}^{n}\right)\right)$ donc converge vers l'unique solution $u$ de (3.1) dans $C\left([0, T] ; L^{2}\left(\mathbb{R}^{n}\right)\right)$ pour tout $u_{0}$ dans $L^{2}\left(\mathbb{R}^{n}\right)$.

\subsection{Le cas non linéaire}

On considère le problème de Cauchy suivant :

$$
\left\{\begin{array}{l}
\partial_{t} u=i \mathscr{L} u+F\left(u, \nabla_{x} u, \bar{u}, \nabla_{x} \bar{u}\right), \quad t \in \mathbb{R}, x \in \mathbb{R}^{n}, \\
u(x, 0)=u_{0}(x) \in H^{s}\left(\mathbb{R}^{n} ; \mathbb{C}\right),
\end{array}\right.
$$

où $F(u, v, \bar{u}, \bar{v})$ est une fonction $C^{\infty}$ de $\mathbb{C} \times \mathbb{C}^{n} \times \mathbb{C} \times \mathbb{C}^{n}$ dans $\mathbb{C}$, nulle en 0 ainsi que ses dérivées d'ordre 1 et 2 . On appelle équation paralinéaire associée, l'équation ci-dessous obtenue par la formule de Bony

$$
\left\{\begin{array}{l}
\partial_{t} u=i \mathscr{L} u+T_{\partial_{v} F} \cdot \nabla_{x} u+T_{\partial_{\bar{v}} F} \cdot \nabla_{x} \bar{u}+T_{\partial_{u} F} \cdot u+T_{\partial_{\bar{u}} F} \cdot \bar{u}+R\left(u, \nabla_{x} u, \bar{u}, \nabla_{x} \bar{u}\right), \\
u(x, 0)=u_{0}(x),
\end{array}\right.
$$

où $R\left(u, \nabla_{x} u, \bar{u}, \nabla_{x} \bar{u}\right) \in H^{n / 2+2 \varrho}$ si $u \in H^{s}$ avec $s=n / 2+1+\varrho$ avec $\varrho>0$. On pose $v=\nabla_{x} u, z_{0}=\left(u_{0}, v_{0}, \bar{u}_{0}, \bar{v}_{0}\right), z=(u, v, \bar{u}, \bar{v}), b_{1}(x)=\partial_{v} F(z), b_{2}(x)=$ $\partial_{\bar{v}} F(z), a_{1}(x)=\partial_{u} F(z), a_{2}(x)=\partial_{\bar{u}} F(z)$ et $b_{1}^{0}(x)=\partial_{v} F\left(z_{0}\right), b_{2}^{0}(x)=\partial_{\bar{v}} F\left(z_{0}\right)$, $a_{1}^{0}(x)=\partial_{u} F\left(z_{0}\right), a_{2}^{0}(x)=\partial_{\bar{u}} F\left(z_{0}\right)$.

L'équation (3.13) s'écrit alors, en posant $v=\nabla_{x} u$ pour simplifier,

$$
\left\{\begin{array}{l}
\partial_{t} u=i \mathscr{L} u+T_{b_{1}^{0}}^{1 / 2} \cdot \nabla_{x} u+T_{b_{2}^{0}}^{1 / 2} \cdot \nabla_{x} \bar{u}+T_{a_{1}^{0}}^{1 / 2} u+T_{a_{2}^{0}}^{1 / 2} \bar{u}+\widetilde{R}_{u}, \\
u(x, 0)=u_{0}(x),
\end{array}\right.
$$


avec $\widetilde{R}_{u}=R(u, v, \bar{u}, \bar{v})+\widetilde{R}_{u, 2}+\widetilde{R}_{u, 3}+\widetilde{R}_{u, 4}$ où

$$
\begin{aligned}
\widetilde{R}_{u, 2} & =\left(T_{b_{1}^{0}}-T_{b_{1}^{0}}^{1 / 2}\right) \cdot v+\left(T_{b_{2}^{0}}-T_{b_{2}^{0}}^{1 / 2}\right) \cdot \bar{v}, \\
\widetilde{R}_{u, 3} & =\left(T_{b_{1}}-T_{b_{1}^{0}}\right) \cdot \nabla u+\left(T_{b_{2}}-T_{b_{2}^{0}}\right) \cdot \nabla \bar{u}, \\
\widetilde{R}_{u, 4} & =\left(T_{a_{1}}-T_{a_{1}^{0}}^{1 / 2}\right) u+\left(T_{a_{2}}-T_{a_{2}^{0}}^{1 / 2}\right) \bar{u} .
\end{aligned}
$$

Dans la suite $A_{n}\left(\left\|u_{0}\right\|_{s}\right)$ et $B_{n}\left(\left\|u_{0}\right\|_{s}\right)$ désignent des constantes qui ne dépendent que de $n, s$ et de $\left\|u_{0}\right\|_{s}$.

Soit $\varrho$ tel que $2<\varrho<s-(n / 2+1)$. Soit $\left(q_{\mu}\right)_{\mu}$ une partition de l'unité subordonnée au $Q_{\mu}$. On a $b_{i}^{0}(x)=\sum_{\mu} q_{\mu} b_{i}^{0}(x)$. Posons $\alpha_{i, \mu}=\left\|q_{\mu} b_{i}^{0}\right\|_{C}$ et $\varphi_{i, \mu}(x)=$ $q_{\mu} b_{i}^{0}(x) /\left\|q_{\mu} b_{i}^{0}\right\|_{C} e$. Sous les hypothèses du théorème 1.1 ( $F$ est nulle en 0 , ainsi que ses dérivées d'ordre 1 et 2), en utilisant le développement de Taylor avec reste intégrale en 0 à l'ordre 2 de $\partial_{v} F(u, v, \bar{u}, \bar{v})$, en posant $z_{0}=\left(u_{0}, v_{0}, \bar{u}_{0}, \bar{v}_{0}\right)$, on obtient

$$
b_{1}^{0}(x)=\sum_{\gamma \in \mathbb{N}^{2 n+2},|\gamma|=2} z_{0}^{\gamma} h_{1, \gamma}\left(z_{0}\right)
$$

avec $h_{1, \gamma}\left(u_{0}, v_{0}, \bar{u}_{0}, \bar{v}_{0}\right)$ est bornée sur $\mathbb{R}^{n} \operatorname{par} \sup _{x \in\left[-\left\|u_{0}\right\|_{s},\left\|u_{0}\right\|_{s}\right]^{2 n+2}}\left|G_{1, \gamma}(x)\right|$ où

$$
G_{1, \gamma}\left(\mathscr{R}\left(u_{0}\right), \nabla \mathscr{R}\left(u_{0}\right), \mathscr{I}\left(u_{0}\right), \nabla \mathscr{I}\left(u_{0}\right)\right)=h_{1, \gamma}\left(u_{0}, v_{0}, \bar{u}_{0}, \bar{v}_{0}\right) .
$$

On a le même résultat pour $b_{2}$ mais en utilisant $\partial_{\bar{v}} F(u, v, \bar{u}, \bar{v})$.

D'après l'inégalité de Sobolev, sachant que sur le support de $q_{\mu}$ on a $\left\langle x-x_{\mu}\right\rangle \leq$ $d_{n}$ et que $s-1>n / 2+\varrho$, en posant $\iota_{\mu}=\left\langle x-x_{\mu}\right\rangle^{-(n+1)}$, on obtient

$$
\sup _{i \in\{1,2\}} \alpha_{i, \mu} \leq A_{n}\left(\left\|u_{0}\right\|_{s}\right)\left\|\iota_{\mu}^{2} u_{0} v_{0}\right\|_{s-1} \leq A_{n}\left(\left\|u_{0}\right\|_{s}\right)\left\|\iota_{\mu} u_{0}\right\|_{s-1}\left\|\iota_{\mu} v_{0}\right\|_{s-1} .
$$

D'après le lemme 2.5, on a

$$
\left\|\iota_{\mu} v_{0}\right\|_{s-1} \in l^{1} \quad \text { et } \quad \sup _{i \in\{1,2\}}\|\| \iota_{\mu} b_{i}^{0}\left\|_{s-1}\right\|_{l^{1}} \leq B_{n}\left(\left\|u_{0}\right\|_{s}\right)
$$

donc $\left(\alpha_{i, \mu}\right) \in l^{1}$ avec $\sum_{\mu} \alpha_{i, \mu} \leq B_{n}\left(\left\|u_{0}\right\|_{s}\right)$.

On peut donc appliquer le théorème 3.1 à l'équation

$$
\left\{\begin{array}{l}
\partial_{t} u=i \mathscr{L} u+T_{b_{1}^{0}}^{1 / 2} \cdot \nabla u+T_{b_{2}^{0}}^{1 / 2} \cdot \nabla \bar{u}+T_{a_{1}^{0}}^{1 / 2} u+T_{a_{2}^{0}}^{1 / 2} \bar{u}+f \text { où } f=\widetilde{R}_{u} \\
u(x, 0)=u_{0}(x) .
\end{array}\right.
$$

On définit $\lambda_{1}^{T}(w), \lambda_{2}^{T}(w)$ et $\lambda_{3}^{T}(w)$ par

$$
\begin{array}{cl}
\lambda_{1}^{T}(w)=\sup _{[0, T]}\|w\|_{s}, & \lambda_{2}^{T}(w)=\|\| J^{s+1 / 2} w\left\|_{T}, \quad \lambda_{3}^{T}(w)=\sup _{[0, T]}\right\| \partial_{t} w \|_{n / 2+1+\varepsilon}, \\
\Gamma^{T}(w) & =\max \left(\lambda_{1}^{T}(w), \lambda_{2}^{T}(w), \lambda_{3}^{T}(w)\right) .
\end{array}
$$

On pose

$$
Z_{\left\|u_{0}\right\|_{s}}^{T}=\left\{w \in C\left([0, T] ; H^{s}\left(\mathbb{R}^{n}\right)\right): w(x, 0)=u_{0}(x) \text { et } \Gamma^{T}(w) \leq 10 K_{n}\left(\left\|u_{0}\right\|_{s}\right)\right\},
$$


où $K_{n}\left(\left\|u_{0}\right\|_{s}\right)$ désigne une constante fixé assez grande ne dépendant que de $n$, de $s$ et de $\left\|u_{0}\right\|_{s}$ telle que $K_{n}\left(\left\|u_{0}\right\|_{s}\right)=\left\|u_{0}\right\|_{s} C_{n}\left(\left\|u_{0}\right\|_{s}\right)$ avec $C_{n}\left(\left\|u_{0}\right\|_{s}\right)$ une constante polynomiale en $n, B_{n}\left(\left\|u_{0}\right\|_{s}\right)$ et $e^{B_{n}\left(\left\|u_{0}\right\|_{s}\right)}$.

Dans la suite, pour tout $w \in Z_{\left\|u_{0}\right\|_{s}}^{T}$, on pose

$$
\Upsilon w(t)=W(t) u_{0}+\int_{0}^{t} W\left(t-t^{\prime}\right) \widetilde{R}_{w}\left(t^{\prime}\right) d t^{\prime},
$$

où $W(t) u_{0}$ est la solution de l'équation (3.15) pour $f=0$ (l'existence énoncée dans le théorème 3.1 n'est utilisée que dans le cas $f=0$ ). Un point fixe de $\Upsilon$ est solution de (3.14) et donc solution du problème de Cauchy (3.12). En effet, en notant $B$ l'opérateur conjugaison, on a

$$
\begin{aligned}
& \partial_{t} \Upsilon w(t)=\left(i \mathscr{L}+T_{b_{1}^{0}}^{1 / 2} \cdot \nabla+T_{b_{2}^{0}}^{1 / 2} \cdot \nabla B+T_{a_{1}^{0}}^{1 / 2}+T_{a_{2}^{0}}^{1 / 2} B\right) W(t) u_{0} \\
&+\int_{0}^{t}\left(i \mathscr{L}+T_{b_{1}^{0}}^{1 / 2} \cdot \nabla+T_{b_{2}^{0}}^{1 / 2} \cdot \nabla B+T_{a_{1}^{0}}^{1 / 2}+T_{a_{2}^{0}}^{1 / 2} B\right) W\left(t-t^{\prime}\right) \widetilde{R}_{w}\left(t^{\prime}\right) d t^{\prime}+\widetilde{R}_{w}(t) \\
&(3.16) \quad=\left(i \mathscr{L}+T_{b_{1}^{0}}^{1 / 2} \cdot \nabla+T_{b_{2}^{0}}^{1 / 2} \cdot \nabla B+T_{a_{1}^{0}}^{1 / 2}+T_{a_{2}^{0}}^{1 / 2} B\right) \Upsilon w(t)+\widetilde{R}_{w}(t) .
\end{aligned}
$$

Sachant que $\Upsilon w$ est solution de (3.16), on peut appliquer les estimations (3.2) et (3.3) pour obtenir que

$$
\begin{aligned}
\lambda_{1}^{T}(\Upsilon w)^{2}+\lambda_{2}^{T}(\Upsilon w)^{2} \leq & C_{n}\left(\left\|u_{0}\right\|_{s}\right)^{2}\left(\left\|u_{0}\right\|^{2}+I_{T}\left(R(w, \nabla w, \bar{w}, \nabla \bar{w})+\widetilde{R}_{w, 4}, \Upsilon w\right)\right. \\
& \left.+I_{T}\left(\widetilde{R}_{w, 2}, \Upsilon w\right)+I_{T}\left(\widetilde{R}_{w, 3}, \Upsilon w\right)\right) .
\end{aligned}
$$

Lemme 3.10. Soit $I_{T}(f, u)$ une somme trois termes de la forme $\int_{0}^{T}|\langle G f, u\rangle| d t$ avec $G \in O p S_{0,0}^{0}$ et $\|G\|_{\mathscr{L}\left(L^{2}\left(R^{n}\right)\right.} \leq A$. Pour tout $T>0$, on a

$$
I_{T}(f, u) \leq A T\left(\sup _{[0, T]}\|f\|_{0}^{2}+\sup _{[0, T]}\|u\|_{0}^{2}\right) .
$$

Dans la suite $\theta_{1}$ désigne une fonction dans $C^{\infty}\left(\mathbb{R}^{n}\right)$ telle que $\theta_{1}(x)=0$ si $|x| \leq 1$ et $\theta_{1}(x)=1$ si $|x| \geq 2$.

Si $f=\sum_{\mu} \alpha_{\mu} f_{\mu}$ avec $\sum_{\mu}\left|\alpha_{\mu}\right| \leq A$ alors, pour tout $T>0$, tout $R^{\prime} \geq 1$ et tout $R \geq 1$, on $a$

$I_{T}\left(J^{s} f, J^{s} u\right) \leq A R^{\prime} \sup _{\mu}\left\|\left\langle x-x_{\mu}\right\rangle^{2} J^{s-1 / 2} \theta_{1}(D / R) f_{\mu}\right\|_{L^{2}\left(\mathbb{R}^{n} \times[0, T]\right)}^{2}+\frac{A}{R^{\prime}}\left\|\mid J^{s+1 / 2} u\right\| \|_{T}^{2}$

$$
+A T\left(\sup _{[0, T]}\left\|\left(\operatorname{Id}-\theta_{1}(D / R)\right) J^{s} f\right\|_{0}^{2}+\sup _{[0, T]}\left\|J^{s} u\right\|_{0}^{2}\right) .
$$

Cette dernière estimation est aussi vérifiée avec Id au lieu de $\theta_{1}(D / R)$.

Preuve : Voir section 4.

On applique alors (3.17) à $I_{T}\left(R(w, \nabla w, \bar{w}, \nabla \bar{w})+\widetilde{R}_{w, 4}, \Upsilon w\right)$. De plus, en posant

$$
b_{3, i}(x, t)=\int_{0}^{1} \partial_{t} b_{i}\left(x, t t^{\prime}\right) d t^{\prime},
$$


on a $T_{b_{i}}-T_{b_{i}^{0}}=t T_{b_{3, i}(x, t)}$. On a aussi

$$
\partial_{t} b_{i}(x, t)=\sum_{\mu} \widetilde{\alpha}_{i, \mu} \widetilde{\widetilde{\varphi}}_{i, \mu}
$$

avec $\widetilde{\alpha}_{i, \mu}=\left\|q_{\mu} \partial_{t} b_{i}(x, t)\right\|_{L^{\infty}\left(\mathbb{R}^{n}\right)}$ et $\widetilde{\widetilde{\varphi}}_{i, \mu}=q_{\mu} \partial_{t} b_{i} /\left\|q_{\mu} \partial_{t} b_{i}(x, t)\right\|_{L^{\infty}\left(\mathbb{R}^{n}\right)}$.

Sachant que $w \in Z_{\left\|u_{0}\right\|_{s}}^{T}$, que, par exemple pour $i=1$,

$$
b_{i}(x, t)=\sum_{\gamma \in \mathbb{N}^{2 n+2},|\gamma|=2} z^{\gamma} h_{1, \gamma}(z),
$$

avec $z=\left(w, \nabla_{x} w, \bar{w}, \nabla_{x} \bar{w}\right)$, pour $s_{2}=n / 2+1+\varepsilon$, on a

$$
\partial_{t} b_{i} \in H^{n / 2+\varepsilon} \quad \text { et } \quad \sum_{\mu}\left|\widetilde{\alpha}_{i, \mu}\right| \leq A_{n}\left(\sup _{[0, T]}\|w\|_{s_{2}}\right)\left\|\partial_{t} w\right\|_{s_{2}} \leq D_{n}\left(\left\|u_{0}\right\|_{s}\right) \text {. }
$$

On peut donc appliquer (3.18) à $I_{T}\left(\widetilde{R}_{w, 2}, \Upsilon w\right)$ et à $I_{T}\left(\widetilde{R}_{w, 3}, \Upsilon w\right)$.

Pour $R$ et $R^{\prime}$ assez grand et $T$ assez petit, en posant $b_{i, \mu}=\int_{0}^{1} \widetilde{\widetilde{\varphi}}_{i, \mu}\left(x, t t^{\prime}\right) d t^{\prime}$, on obtient donc

$$
\begin{aligned}
\lambda_{1}^{T}(\Upsilon w)^{2}+ & \lambda_{2}^{T}(\Upsilon w)^{2} \\
\leq & C_{n}\left(\left\|u_{0}\right\|_{s}\right)^{2}\left(\left\|u_{0}\right\|_{s}^{2}+T \sup _{[0, T]} \| R\left(w, \nabla w, \bar{w}, \nabla \bar{w}+\widetilde{R}_{w, 4} \|_{s}^{2}\right.\right. \\
& +T D_{n}\left(\left\|u_{0}\right\|_{s}\right) \sup _{i, \mu}\left(\int_{0}^{T}\left\|\left\langle x-x_{\mu}\right\rangle^{2} J^{s-1 / 2} T_{b_{i, \mu}} \cdot \nabla w\right\|_{0}^{2} d t\right) \\
& \left.+\sup _{i, \mu}\left(\int_{0}^{T} \|\left\langle x-x_{\mu}\right\rangle^{2} J^{s-1 / 2} \theta_{1}\left(\frac{D}{R}\right)\left(T_{\varphi_{i, \mu}^{0}}-T_{\varphi_{i, \mu}^{0}}^{1 / 2}\right) . \nabla w\right) \|_{0}^{2} d t\right) .
\end{aligned}
$$

D'après le théorème 2.10 et la proposition 2.16 , pour $\varrho>1 / 2$, on a

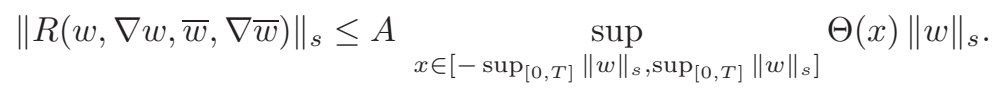

On rappelle que, par construction, $\left\|b_{\mu}\right\|_{L^{\infty}} \leq 1$ et $\left\|\varphi_{i, \mu}\right\|_{C} \leq \leq 1$.

De plus, on a les deux lemmes suivants.

Lemme 3.11. On a

$$
\sup _{i, \mu}\left(\int_{0}^{T}\left\|\left\langle x-x_{\mu}\right\rangle^{2} J^{s-1 / 2} T_{b_{i, \mu}} \cdot \nabla w\right\|_{0}^{2} d t\right)^{1 / 2} \leq A\left(\lambda_{2}^{T}(w)+T \lambda_{1}^{T}(w)\right) .
$$

\section{Lemme 3.12.}

$$
\begin{aligned}
& \sup _{i, \mu}\left(\int_{0}^{T} \|\left\langle x-x_{\mu}\right\rangle^{2} J^{s-1 / 2} \theta_{1}\left(\frac{D}{R}\right)\right.\left.\left(T_{\varphi_{i, \mu}^{0}}-T_{\varphi_{i, \mu}^{0}}^{1 / 2}\right) . \nabla w \|_{0}^{2} d t\right)^{1 / 2} \\
& \leq A\left(R T \lambda_{1}^{T}(w)+\frac{1}{R^{1 / 2}} \lambda_{2}^{T}(w)\right) .
\end{aligned}
$$

Preuve : Voir section 4. 
Sachant que $R^{-1} \leq R^{-1 / 2}$, on obtient donc que

$$
\begin{aligned}
\lambda_{1}^{T}(\Upsilon w)^{2}+\lambda_{2}^{T}(\Upsilon w)^{2} \leq & C_{n}\left(\left\|u_{0}\right\|_{s}\right)^{2}\left(\left\|u_{0}\right\|_{s}^{2}+D_{n}\left(\left\|u_{0}\right\|_{s}\right)\left(\left(R^{-1 / 2}+T\right) \lambda_{2}^{T}(w)^{2}\right.\right. \\
& \left.\left.+A(1+R) T \lambda_{1}^{T}(w)^{2}\right)\right) .
\end{aligned}
$$

Ce qui donne, pour $R$ assez grand et $T$ assez petit,

$$
\lambda_{1}^{T}(\Upsilon w)+\lambda_{2}^{T}(\Upsilon w) \leq 2 K_{n}\left(\left\|u_{0}\right\|_{s}\right) .
$$

Estimons alors $\lambda_{3}^{T}(\Upsilon w)=\sup _{[0, T]}\left\|\partial_{t} \Upsilon w\right\|_{n / 2+1+\varepsilon}$. On rappelle que $\Upsilon w$ est solution de (3.15) avec $f=\widetilde{R}_{w}$ et $\Upsilon w(0)=u_{0}$. En écrivant que

$$
\begin{aligned}
f= & \widetilde{R}\left(u_{0}, \nabla_{x} u_{0}, \bar{u}_{0}, \nabla_{x} \bar{u}_{0}\right)+\widetilde{R}\left(w, \nabla_{x} w, \bar{w}, \nabla_{x} \bar{w}\right)-\widetilde{R}\left(u_{0}, \nabla_{x} u_{0}, \bar{u}_{0}, \nabla_{x} \bar{u}_{0}\right) \\
& +\left(T_{b_{1}}^{\delta}-T_{b_{1}^{0}}^{\delta}\right) \cdot \nabla w+\left(T_{b_{2}}^{\delta}-T_{b_{2}^{0}}^{\delta}\right) \cdot \nabla \bar{w}+\left(T_{a_{1}}^{\delta}-T_{a_{1}^{0}}^{\delta}\right) w+\left(T_{a_{2}}^{\delta}-T_{a_{2}^{0}}^{\delta}\right) \bar{w}
\end{aligned}
$$

et que, en posant $w_{R}=\left(1-\theta_{1}(D / R)\right) w$ et $u_{R}=\left(1-\theta_{1}(D / R)\right) u_{0}$ où $R$ est fixé assez grand, on a

$$
\begin{aligned}
& \widetilde{R}\left(w, \nabla_{x} w, \bar{w}, \nabla_{x} \bar{w}\right)-\widetilde{R}\left(u_{0}, \nabla_{x} u_{0}, \bar{u}_{0}, \nabla_{x} \bar{u}_{0}\right) \\
&= \widetilde{R}\left(w, \nabla_{x} w, \bar{w}, \nabla_{x} \bar{w}\right)-\widetilde{R}\left(w_{R}, \nabla_{x} w_{R}, \bar{w}_{R}, \nabla_{x} \bar{w}_{R}\right) \\
&+\widetilde{R}\left(w_{R}, \nabla_{x} w_{R}, \bar{w}_{R}, \nabla_{x} \bar{w}_{R}\right)-\widetilde{R}\left(u_{R}, \nabla_{x} u_{R}, \bar{u}_{R}, \nabla_{x} \bar{u}_{R}\right) \\
&+\widetilde{R}\left(u_{R}, \nabla_{x} u_{R}, \bar{u}_{R}, \nabla_{x} \bar{u}_{R}\right)-\widetilde{R}\left(u_{0}, \nabla_{x} u_{0}, \bar{u}_{0}, \nabla_{x} \bar{u}_{0}\right),
\end{aligned}
$$

en utilisant alors la proposition 2.16 et le théorème 2.10 , on obtient que

$$
\lambda_{3}^{T}(\Upsilon w) \leq \lambda_{1}^{T}(\Upsilon w)+E_{n}\left(\left\|u_{0}\right\|_{s}\right)\left(T R^{2}+R^{-1}\right)+K_{n}\left(\left\|u_{0}\right\|_{s}\right)
$$

avec $E_{n}\left(\left\|u_{0}\right\|_{s}\right)$ est une constante fixée assez grande.

On utilise alors l'estimation (3.20), ce qui donne, pour $R$ assez grand et $T$ assez petit,

$$
\lambda_{3}^{T}(\Upsilon w) \leq 10 K_{n}\left(\left\|u_{0}\right\|_{s}\right) .
$$

On déduit de (3.20) et de (3.21) que $\Upsilon\left(Z_{\left\|u_{0}\right\|_{s}}^{T}\right) \subset Z_{\left\|u_{0}\right\|_{s}}^{T}$.

Pour prouver que, pour $T$ assez petit, $\Upsilon$ est contractante sur $Z_{\left\|u_{0}\right\|_{s}}^{T}$, comme précédemment, on applique les estimations du théorème 3.1 à $\Upsilon w_{1}-\Upsilon w_{2}$, ce qui est possible car

$$
\begin{aligned}
& \partial_{t}\left(\Upsilon w_{1}-\Upsilon w_{2}\right) \\
& \quad=\left(i \mathscr{L}+T_{b_{1}^{0}}^{1 / 2} \cdot \nabla+T_{b_{2}^{0}}^{1 / 2} \cdot \nabla B+T_{a_{1}^{0}}^{1 / 2}+T_{a_{2}^{0}}^{1 / 2} B\right)\left(\Upsilon w_{1}-\Upsilon w_{2}\right)+\widetilde{R}_{w_{1}}-\widetilde{R}_{w_{2}} .
\end{aligned}
$$

On remarque, par exemple, que

$$
\begin{aligned}
\widetilde{R}_{w_{1}, 3}-\widetilde{R}_{w_{2}, 3}= & \left(T_{b_{1}\left(w_{1}\right)}-T_{b_{1}\left(w_{2}\right)}\right) \cdot \nabla_{x} w_{1}+T_{b_{1}\left(w_{2}\right)} \cdot \nabla_{x}\left(w_{1}-w_{2}\right) \\
& +\left(T_{b_{1}\left(w_{1}\right)}-T_{b_{1}\left(w_{2}\right)}\right) \cdot \nabla_{x} \overline{w_{1}}+T_{b_{1}\left(w_{2}\right)} \cdot \nabla_{x}\left(\overline{w_{1}-w_{2}}\right),
\end{aligned}
$$


sachant que

$$
b_{1}\left(w_{1}\right)-b_{1}\left(w_{2}\right)=\left(w_{1}-w_{2}\right) \cdot \int_{0}^{1}\left(\nabla b_{1}\right)\left(t^{\prime} w_{1}+\left(1-t^{\prime}\right) w_{2}\right) d t^{\prime}
$$

le second membre de l'égalité ci-dessus admettant une décomposition $\sum_{\mu} \alpha_{4, \mu} \widetilde{\varphi}_{4, \mu}$ suivant les $Q_{\mu}$ telle que

$$
\sum_{\mu}\left|\alpha_{4, \mu}\right| \leq \sup _{[0, T]}\left\|w_{1}-w_{2}\right\|_{n / 2+\varrho^{\prime}} \sup _{t^{\prime} \in[0,1], t \in[0, T]}\left\|\left(\nabla b_{1}\right)\left(t^{\prime} w_{1}+\left(1-t^{\prime}\right) w_{2}\right)\right\|_{n / 2+\varrho^{\prime}} .
$$

On utilise aussi une nouvelle fois le théorème 2.10 pour estimer

$$
\left\|R\left(w_{1}, \nabla_{x} w_{1}, \bar{w}_{1}, \nabla_{x} \bar{w}_{1}\right)-R\left(w_{2}, \nabla_{x} w_{2}, \bar{w}_{2}, \nabla_{x} \bar{w}_{2}\right)\right\|_{s}
$$

et, les arguments déjà utilisés pour les autres termes, on obtient que, pour tout $w_{1}$ et tout $w_{2}$ dans $Z_{\left\|u_{0}\right\|_{s}}^{T}$,

$$
\Gamma^{T}\left(\Upsilon w_{1}-\Upsilon w_{2}\right) \leq F_{n}\left(\left\|u_{0}\right\|_{s}\right)\left(T+R^{-\delta \varrho}\right) \Gamma^{T}\left(w_{1}-w_{2}\right) .
$$

Ce qui permet d'obtenir que $\Upsilon$ est contractante dans $Z_{\left\|u_{0}\right\|_{s}}^{T}$ pour $R$ assez grand et $T$ assez petit.

Sachant que $Z_{\left\|u_{0}\right\|_{s}}^{T}$ est un espace métrique complet, on peut donc appliquer le théorème du point fixe. On obtient alors le théorème 1.1 car

$$
Z_{\left\|u_{0}\right\|_{s}}^{T} \subset\left\{u \in C\left([0, T] ; H^{s}\left(\mathbb{R}^{n}\right)\right), u(x, 0)=u_{0} \text { et }\left\|\mid J^{s+1 / 2} u\right\|_{T}<\infty\right\}=E_{T} .
$$

L'unicité dans $E_{T}$ s'obtient en remarquant que si une solution $u$ existe dans $E_{T}$ alors, en appliquant les estimations du théorème 3.1 à $u$ et en utilisant des arguments déjà utilisés pour obtenir le point fixe, pour $T>0$ assez petit, on a $u \in Z_{\left\|u_{0}\right\|_{s}}^{T}$.

Pour prouver la propriété de continuité par rapport aux données initiales, on remarque tout d'abord que, s'il existe $r>0$ tel que $\left\|u_{0}\right\| \leq r$ alors toutes le constantes $A_{n}\left(\left\|u_{0}\right\|_{s}\right), B_{n}\left(\left\|u_{0}\right\|_{s}\right), \ldots, K_{n}\left(\left\|u_{0}\right\|_{s}\right)$ se majorent par une constantes $A_{n, r}$ ne dépendant plus de $u_{0}$, et donc la solution $u$ associée à $u_{0}$ existe sur un intervalle $\left[-T_{r}, T_{r}\right]$ avec $T_{r}$ indépendant de $u_{0}$. On utilise ensuite que $u-\widetilde{u}$ est solution de (3.15) avec

$$
f=\widetilde{R}_{u}-\widetilde{R}_{\widetilde{u}}+T_{b_{1}^{0}-b_{1}\left(\widetilde{u}_{0}\right)} \cdot \nabla_{x} \widetilde{u}+T_{b_{2}^{0}-b_{2}\left(\widetilde{u}_{0}\right)} \cdot \nabla_{x} \overline{\widetilde{u}}+T_{a_{1}^{0}-a_{1}\left(\widetilde{u}_{0}\right)} \widetilde{u}+T_{a_{2}^{0}-a_{2}\left(\widetilde{u}_{0}\right)} \overline{\widetilde{u}}
$$

où $b_{1}\left(\widetilde{u}_{0}\right)=\left(\nabla_{v} F\right)\left(\widetilde{u}_{0}, \nabla \widetilde{u}_{0}, \overline{\widetilde{u}}_{0}, \nabla \overline{\widetilde{u}}_{0}\right)$, etc. On obtient alors que, pour $T=T_{r}$, on a

$$
\Gamma^{T_{r}}(u-\widetilde{u}) \leq 10 C_{n}\left(\left\|u_{0}-\widetilde{u}_{0}\right\|_{s}\right)\left\|u_{0}-\widetilde{u}_{0}\right\|_{s}+\left\|u_{0}-\widetilde{u}_{0}\right\|_{s} T_{r} H_{n}\left(\left\|\widetilde{u}_{0}\right\|_{s}\right) .
$$

Cette dernière estimation permet d'obtenir l'uniforme continuité par rapport aux données initiales pour $T=T_{r}$ assez petit. 


\section{Preuve des lemmes}

\subsection{Preuve du lemme 3.3}

La preuve de ce lemme suit le même raisonnement que pour celle du lemme 3.3 dans [5]. Les modifications à apporter sont dues au fait que l'on travaille avec des opérateurs $T_{b}^{\delta}$ de symboles $\widetilde{b}^{\delta}(x, \xi)$ (notés dans cette démonstration $T_{b}$ et $\widetilde{b}(x, \xi)$, la valeur de $\delta<1$ ne modifiant pas la démonstration.) au lieu de $b(x)$. Dans la suite, on désigne par $\widetilde{C}_{1}$ et $\widetilde{C}_{2}$ les opérateurs tels que $\widetilde{C}_{i} \bar{u}=\overline{C_{i} u}$. On a donc $\widetilde{C}_{i} u=\overline{C_{i} \bar{u}}$ or, pour tout réel $s, C_{i}$ est borné de $H^{s}$ dans $H^{s}$ donc $\widetilde{C}_{i}$ aussi, ainsi que leurs adjoints respectifs.

La démonstration est écrite simultanément dans le cas $\mathbf{C}=\mathrm{Id}$ et $\mathbf{C} \neq \mathrm{Id}$ en précisant les simplifications éventuelles otenues dans les calculs, la démarche étant la même dans les deux cas.

On a

$$
\begin{aligned}
\partial_{t} \mathbf{C} u= & i \mathscr{L} \mathbf{C} u+i[\mathbf{C}, \mathscr{L}] u+T_{b_{1}} \cdot \nabla_{x} \mathbf{C} u+T_{b_{2}} \cdot \nabla_{x} \overline{\mathbf{C} u}+C_{1} \mathbf{C} u+C_{2} \overline{\mathbf{C} u}+\mathbf{C} f(x, t) \\
& +\left[\mathbf{C}, T_{b_{1}} \cdot \nabla\right] u+\left[\mathbf{C}, T_{b_{2}} \cdot \nabla\right] \bar{u}+\left[\mathbf{C}, C_{1}\right] u+\left[\mathbf{C}, C_{2}\right] \bar{u} .
\end{aligned}
$$

On pose aussi

$$
\begin{gathered}
B=\left(\begin{array}{ll}
T_{b_{1}} \cdot \nabla_{x} & T_{b_{2}} \cdot \nabla_{x} \\
T_{\bar{b}_{2}} \cdot \nabla_{x} & T_{\overline{b_{1}}} \cdot \nabla_{x}
\end{array}\right), \quad \Phi=\left(\begin{array}{ll}
C_{1} & C_{2} \\
\widetilde{C}_{1} & \widetilde{C}_{2}
\end{array}\right), \\
\Psi_{M}=\left(\begin{array}{ll}
\Psi & 0 \\
0 & -\Psi
\end{array}\right), \quad H=\left(\begin{array}{ll}
\mathscr{L} & 0 \\
0 & -\mathscr{L}
\end{array}\right),
\end{gathered}
$$

où $\Psi$ est un opérateur dont on choisira le symbole $\psi(x, \xi)$ dans $S_{1,0}^{0}$ vérifiant $\psi(x, \xi) \geq A$ avec $A>0$. De plus, d'après la proposition (v) du lemme 3.6 (dans la preuve du lemme 3.2 ), on a

$$
i[\mathbf{C}, \mathscr{L}]=-\mathbf{C} T_{i \mathscr{I}\left(b_{1}\right)} \cdot \nabla+E_{1, R}+E_{2, R}+E
$$

donc

$$
\begin{gathered}
i\left(\begin{array}{ll}
{[\mathbf{C}, \mathscr{L}]} & 0 \\
0 & -[\mathbf{C}, \mathscr{L}]
\end{array}\right)=\widetilde{B} \mathbf{C}+g(x, D) \widetilde{I} \text { avec } \\
\widetilde{B}=\left(\begin{array}{ll}
-T_{i \mathscr{I}\left(b_{1}\right)} . \nabla & 0 \\
0 & -T_{\overline{i \mathscr{I}\left(b_{1}\right)}} \cdot \nabla
\end{array}\right), \quad \widetilde{I}=\left(\begin{array}{ll}
\mathrm{Id} & 0 \\
0 & -\mathrm{Id}
\end{array}\right) \text { et } \\
g(x, D)=-\left[\mathbf{C}, T_{i \mathscr{I}\left(b_{1}\right)} \cdot \nabla\right]+E_{1, R}+E_{2, R}+E
\end{gathered}
$$

D'après la proposition (v), mais aussi la proposition (iv) du lemme 3.6, on a

$$
\int_{0}^{T}\langle g(x, D) u, u\rangle d t \leq A \sup _{\mu}\left\|\varphi_{1, \mu}\right\|_{C^{M}}^{N}\left(\frac{1}{R}\|\| J^{1 / 2} u\|\|_{T}^{2}+R T \sup _{[0, T]}\|u\|_{0}^{2}\right) .
$$

Remarque : Dans le cas où $\mathbf{C}=\mathrm{Id}$, le commutateur $[\mathbf{C}, \mathscr{L}]=0$ et donc dans le cas $\mathbf{C}=\mathrm{Id}$, on n'a pas les termes en $\widetilde{B}$ et $g(x, D)$. C'est le terme en $g(x, D)$ qui fait que l'estimation dans le cas $\mathbf{C} \neq$ Id est différente de celle obtenue dans le cas $\mathbf{C}=$ Id. 
En posant $\vec{w}=(u, \bar{u})^{T}$, on a

$$
\partial_{t} \mathbf{C} \vec{w}=i H \mathbf{C} \vec{w}+(B+\widetilde{B}+\Phi) \mathbf{C} \vec{w}+([B+\Phi, \mathbf{C} \mathrm{Id}]+g(x, D) \widetilde{I}) \vec{w}+\mathbf{C} \vec{F}
$$

avec $\vec{F}=(f(x), \overline{f(x)})^{T}$. Sachant que $\mathbf{c}(x, \xi)$ est pair en $\xi$ et réel donc $\mathbf{C} \bar{u}=\overline{\mathbf{C} u}$, en posant dans la suite $\vec{w}_{c}=(\mathbf{C} u, \overline{\mathbf{C} u})$ et $\vec{w}=(u, \bar{u})$, on obtient que

$$
\begin{aligned}
\partial_{t}\left\langle\Psi_{M} \vec{w}_{c}, \vec{w}_{c}\right\rangle= & -i\left\langle\left(H \Psi_{M}-\Psi_{M} H\right) \vec{w}_{c}, \vec{w}_{c}\right\rangle+\left\langle\left(B^{*} \Psi_{M}+\Psi_{M} B\right) \vec{w}_{c}, \vec{w}_{c}\right\rangle \\
& +\left\langle\left(\widetilde{B}^{*} \Psi_{M}+\Psi_{M} \widetilde{B}\right) \vec{w}_{c}, \vec{w}_{c}\right\rangle+\left\langle\left(\Phi^{*} \Psi_{M}+\Psi_{M} \Phi\right) \vec{w}_{c}, \vec{w}_{c}\right\rangle \\
& \left.+\left\langle\Psi_{M} \mathbf{C} \vec{F}, \vec{w}_{c}\right\rangle+\left\langle\Psi_{M} \vec{w}_{c}, \mathbf{C} \vec{F}\right\rangle\right\rangle+\left\langle G \Psi_{M} \vec{w}, \vec{w}\right\rangle+\left\langle\Psi_{M} \vec{w}, G \vec{w}\right\rangle .
\end{aligned}
$$

avec $G=[B+\Phi, \mathbf{C I d}]+g(x, D)$. De plus, modulo des opérateurs bornés dans $L^{2}$, on a

$$
\left\langle\left(B^{*} \Psi_{M}+\Psi_{M} B\right) \vec{w}, \vec{w}\right\rangle=\left\langle\left(\begin{array}{cc}
\Psi T_{b_{1}}-\overline{b_{1}} \cdot \nabla_{x} & 2 \Psi T_{b_{2}} \cdot \nabla_{x} \\
-2 \Psi T_{\overline{b_{2}}} \cdot \nabla_{x} & \Psi T_{b_{1}-\overline{b_{1}}} \cdot \nabla_{x}
\end{array}\right) \vec{w}, \vec{w}\right\rangle
$$

En notant $\psi$ le symbole de $\Psi$,

$$
\sigma\left(B^{*} \Psi_{M}+\Psi_{M} B\right)=\psi(x, \xi)\left(\begin{array}{cc}
i\left(\widetilde{b}_{1}-\overline{\widetilde{b}_{1}}\right)(x, \xi) \cdot \xi & 2 i \widetilde{b}_{2}(x, \xi) \cdot \xi \\
-2 i \widetilde{\widetilde{b}_{2}}(x, \xi) \cdot \xi & i\left(\widetilde{b}_{1}-\widetilde{\widetilde{b}_{1}}\right)(x, \xi) \cdot \xi
\end{array}\right)
$$

De plus

$$
\sigma\left(\widetilde{B}^{*} \Psi_{M}+\Psi_{M} \widetilde{B}\right)=\psi(x, \xi)\left(\begin{array}{cc}
2 \mathscr{I}\left(\widetilde{b}_{1}(x, \xi)\right) \cdot \xi & 0 \\
0 & -2 \mathscr{I}\left(\widetilde{b}_{1}(x, \xi)\right) \cdot \xi
\end{array}\right)
$$

Le terme $\left\langle\Psi_{M}[B+\Phi, \mathbf{C}\right.$ Id $\left.] \vec{w}, \vec{w}\right\rangle+\left\langle\Psi_{M} \vec{w},[B+\Phi, \mathbf{C}\right.$ Id $\left.] \vec{w}\right\rangle$ s'estime comme celui en $g(x, D)$.

Remarque : le commutateur $[B+\Phi, \mathbf{C}$ Id $]$ est nul si $\mathbf{C}=\mathrm{Id}$.

Dans la suite, on utilise le lemme suivant

Lemme 4.1 (Lemme de Doi). Soit $\lambda$ une fonction paire, positive, régulière et bornée ainsi que toutes ses dérivées, $\lambda \in L^{1}(0, \infty)$. Il existe une fonction régulière $\theta_{0}(x)=\left(\theta_{1}(x), \ldots, \theta_{n}(x)\right)$ bornée, ainsi que toutes ses dérivées telle que, au sens des matrices, on ait

$$
D \theta_{\text {symm }}(x)=\frac{1}{2}\left(\partial_{x_{j}} \theta_{k}+\partial_{x_{k}} \theta_{j}(x)\right) \geq \lambda(|x|) I .
$$

On peut choisir $\theta_{0}(x)=\left(f\left(x_{1}\right), \ldots, f\left(x_{n}\right)\right)$ avec $f(t)=\int_{0}^{t} \lambda(|s|) d s$.

On définit $p(x, \xi)=\theta_{0}(x) . \widetilde{\xi} /\langle\xi\rangle \in S_{1,0}^{0}$ où $\widetilde{\xi}=\left(-\xi_{1}, \ldots,-\xi_{k}, \xi_{k+1}, \ldots, \xi_{n}\right)$. On a

$$
-2 \widetilde{\xi} \cdot \nabla_{x} p(x, \xi)=2 \frac{D \theta_{\text {symm }}(x) \widetilde{\xi} \cdot \widetilde{\xi}}{\langle\xi\rangle} \geq \lambda(|x|) \frac{|\xi|^{2}}{\langle\xi\rangle} .
$$

Preuve : Voir [3]. 
D'après le lemme de Doi ci-dessus appliqué avec $\lambda$ que l'on fixera plus tard, en posant,

$$
\gamma(x, \xi)=p\left(x-x_{\mu_{0}}, \xi\right)+10 A_{2} \sum_{\mu \in \mathbb{Z}^{n}}\left(\left|\alpha_{1, \mu}\right|+\left|\alpha_{2, \mu}\right|\right) p\left(x-x_{\mu}, \xi\right),
$$

où $\mu_{0}$ est fixé, $A_{2}=\sup _{\mu, i, x, \xi}\left|\left\langle x-x_{\mu}\right\rangle^{4} \widetilde{\varphi}_{i, \mu}(x, \xi)\right|$, et $p(x, \xi)=-\theta_{0}(x) . \widetilde{\xi} /\langle\xi\rangle$ avec

$$
\theta_{0}(x)=\left(f\left(x_{1}\right), \ldots, f\left(x_{n}\right)\right), \quad f(t)=\int_{0}^{t} \lambda(|s|) d s \quad \text { et } \quad \lambda(|s|)=\langle s\rangle^{-4} .
$$

On obtient donc que $p$ vérifie le lemme de Doi et donc que

$$
-2 \widetilde{\xi} \cdot \nabla_{x} \gamma(x, \xi)
$$

$$
\geq 2 \lambda\left(\left|x-x_{\mu_{0}}\right|\right) \frac{|\xi|^{2}}{\langle\xi\rangle}+\sum_{\mu \in \mathbb{Z}^{n}} 10 A_{2}\left(\left|\alpha_{1, \mu}\right|+\left|\alpha_{2, \mu}\right|\right) \lambda\left(\left|x-x_{\mu}\right|\right) \frac{|\xi|^{2}}{\langle\xi\rangle} .
$$

On choisit $\Psi$ de symbole $\psi(x, \xi)=\exp (-\gamma(x, \xi))$ donc $\psi \in S_{1,0}^{0}$ car $\gamma \in S_{1,0}^{0}$ et $\Psi$ admet une paramétrix dans $S_{1,0}^{0}$.

L'opérateur $-i\left(H \Psi_{M}-\Psi_{M} H\right)+\left(\Psi_{M}(B+\widetilde{B})+(B+\widetilde{B})^{*} \Psi_{M}\right)$ a pour symbole principal la matrice

$$
s(x, \xi)=2 \psi\left(\begin{array}{cc}
\widetilde{\xi} \cdot \nabla_{x} \gamma(x, \xi) & -i \widetilde{b}_{2}(x, \xi) \cdot \xi \\
\overline{i \widetilde{b}_{2}(x, \xi)} \cdot \xi & \widetilde{\xi} \cdot \nabla_{x} \gamma(x, \xi)
\end{array}\right) .
$$

Remarque : Dans le cas $\mathbf{C}=\mathrm{Id}$, on a

$$
s(x, \xi)=2 \psi\left(\begin{array}{cc}
\widetilde{\xi} \cdot \nabla_{x} \gamma(x, \xi)-2 \mathscr{I}\left(\widetilde{b}_{1}(x, \xi)\right) & -i \widetilde{b}_{2}(x, \xi) \cdot \xi \\
\widetilde{i \bar{b}_{2}(x, \xi)} \cdot \xi & \widetilde{\xi} \cdot \nabla_{x} \gamma(x, \xi)-2 \mathscr{I}\left(\widetilde{b}_{1}(x, \xi)\right)
\end{array}\right) .
$$

et les calculs ci-dessous sont écrits dans le cas $\mathbf{C}=$ Id sachant qu'ils sont plus simple dans le cas $\mathbf{C} \neq \mathrm{Id}$ car il n'y a plus le terme en $\mathscr{I}\left(\widetilde{b}_{1}\right)$ mais l'opérateur $\Psi$ construit ci-dessus fait fonctionner la démonstration dans les deux cas.

Définissons alors

$$
\kappa(x, \xi)=\left(\begin{array}{cc}
2 \psi \lambda\left(\left|x-x_{\mu_{0}}\right|\right)|\widetilde{\xi}|^{2} /\langle\xi\rangle & 0 \\
0 & 2 \psi \lambda\left(\left|x-x_{\mu_{0}}\right|\right)|\widetilde{\xi}|^{2} /\langle\xi\rangle
\end{array}\right)
$$

et $\zeta(x, \xi)=-s(x, \xi)-\kappa(x, \xi)$. On a

$$
\begin{aligned}
& \zeta(x, \xi)+\zeta(x, \xi)^{*} \\
& \quad=-4 \psi(x, \xi)\left(\begin{array}{cc}
\widetilde{\xi} \cdot \nabla_{x} \gamma(x, \xi)-\mathscr{I}\left(\widetilde{b}_{1}(x, \xi)\right) & i \widetilde{b}_{2}(x, \xi) \cdot \xi \\
-i{\widetilde{\tilde{b}_{2}}}_{2}(x, \xi) \cdot \xi & \widetilde{\xi} \cdot \nabla_{x} \gamma(x, \xi)-\mathscr{I}\left(\widetilde{b}_{1}(x, \xi)\right)
\end{array}\right)-2 \kappa(x, \xi) .
\end{aligned}
$$

En posant, dans le cas $\mathbf{C}=\mathrm{Id}$,

$$
c=-\widetilde{\xi} \cdot \nabla_{x} \gamma(x, \xi)+2 \mathscr{I}\left(\widetilde{b}_{1}(x, \xi)\right) \cdot \xi-\lambda\left(\left|x-x_{\mu_{0}}\right|\right) \frac{|\widetilde{\xi}|^{2}}{\langle\xi\rangle}
$$


et, dans le cas $\mathbf{C} \neq \mathrm{Id}$,

$$
c=-\widetilde{\xi} \cdot \nabla_{x} \gamma(x, \xi)-\lambda\left(\left|x-x_{\mu_{0}}\right|\right) \frac{|\widetilde{\xi}|^{2}}{\langle\xi\rangle},
$$

on a

$$
\operatorname{det}\left(\zeta(x, \xi)+\zeta(x, \xi)^{*}-\nu \mathrm{Id}\right)=16 \psi^{2}\left(\nu^{2}-2 c \nu+c^{2}-4\left|\widetilde{b}_{2}(x, \xi) \cdot \xi\right|^{2}\right) .
$$

Le discriminant réduit $\Delta$ de ce trinôme du second degré est $4\left|\widetilde{b}_{2}(x, \xi) \cdot \xi\right|^{2}$. Les deux racines réelles du trinôme du seconde $\operatorname{degré~} \operatorname{det}\left(\zeta(x, \xi)+\zeta(x, \xi)^{*}-\nu \mathrm{Id}\right)$ sont donc $\nu_{1}=c-\sqrt{\Delta}$ et $\nu_{2}=\nu_{1}+2 \sqrt{\Delta}$. Il suffit donc de prouver que $\nu_{1} \geq 0$. Pour cela, on utilise le lemme Doi et que, pour $|\xi| \geq 1$, on a

$$
2\left|\widetilde{b}_{1}(x, \xi) \cdot \xi\right|+2\left|\widetilde{b}_{2}(x, \xi) \cdot \xi\right| \leq 4 A_{2} \sum_{\mu}\left\langle x-x_{\mu}\right\rangle^{-4}\left(\left|\alpha_{1, \mu}\right|+\left|\alpha_{2, \mu}\right|\right) \frac{|\xi|^{2}}{\langle\xi\rangle} .
$$

Sachant que $\widetilde{b}_{1} \in C^{2} S_{1,0}^{0}$ donc $\zeta \in C^{2} S_{1,0}^{1}$, d'après l'inégalité de Gårding précisée pour un système, on obtient

$\mathscr{R}\left(\left\langle\left(-i\left(H \Psi_{M}-\Psi_{M} H\right)+\Psi_{M}(B+\widetilde{B})+(B+\widetilde{B})^{*} \Psi_{M}+\kappa(x, D)\right) \vec{w}_{c}, \vec{w}_{c}\right\rangle\right) \leq A\left\|\vec{w}_{c}\right\|_{0}^{2}$

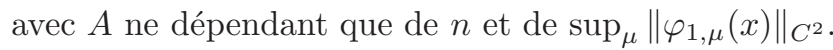

En intégrant alors en temps la partie réelle de (4.2) puis en utilisant l'inégalité précédente sachant que

$$
\kappa(x, D)=\lambda\left(x-x_{\mu_{0}}\right) J \Psi \mathrm{Id}+R_{3}, \quad \text { où } \Psi=\widetilde{\Psi}^{2}+R_{4}
$$

avec $R_{3}$ dans $O p S_{1,0}^{0}, R_{4}$ dans $O p S_{1,0}^{-1}$ et $\widetilde{\Psi}=\exp (-\gamma(x, D) / 2)$, on obtient

$$
\begin{gathered}
\mathscr{R} \int_{0}^{T}\left\langle\sqrt{\lambda}\left(\left|x-x_{\mu_{0}}\right|\right) \widetilde{\Psi} J^{1 / 2} \vec{w}_{c}, \sqrt{\lambda}\left(\left|x-x_{\mu_{0}}\right|\right) \widetilde{\Psi} J^{1 / 2} \vec{w}_{c}\right\rangle d t \\
\leq(A+A T) \sup _{0 \leq t \leq T}\left\|\vec{w}_{c}\right\|^{2}+\mathscr{R} \int_{0}^{T}\left(\left\langle\Psi_{M} \mathbf{C} \vec{F}, \vec{w}_{c}\right\rangle+\left\langle\Psi_{M} \vec{w}_{c}, \mathbf{C} \vec{F}\right\rangle\right) d t \\
+A \sup _{\mu, i}\left\|\varphi_{i, \mu}\right\|_{C^{M}}^{3}\left(\frac{1}{R} \mid\left\|J^{1 / 2} u\right\|\left\|_{T}^{2}+R T \sup _{[0, T]}\right\| u \|_{0}^{2}\right) .
\end{gathered}
$$

De plus, on a

$$
\left|\int_{0}^{T}\left\langle\Psi_{M} \mathbf{C} \vec{F}, \vec{w}_{c}\right\rangle d t\right| \leq 2 \int_{0}^{T} \mathscr{R}(\langle\Psi \mathbf{C} f, \mathbf{C} u\rangle) d t
$$

On a aussi

$$
\begin{aligned}
\mathscr{R} \int_{0}^{T}\left\langle\sqrt{\lambda}\left(\left|x-x_{\mu_{0}}\right|\right) \widetilde{\Psi} J^{1 / 2} \vec{w}_{c}, \sqrt{\lambda}\left(\left|x-x_{\mu_{0}}\right|\right) \widetilde{\Psi} J^{1 / 2} \vec{w}_{c}\right\rangle d t \\
\geq \mathscr{R} \int_{0}^{T} \int_{\mathbb{R}^{n}}\left|\eta\left(\left|x-x_{\mu_{0}}\right|\right) \widetilde{\Psi} J^{1 / 2} \mathbf{C} u\right|^{2} d x d t .
\end{aligned}
$$


En utilisant l'inversibilité de $\widetilde{\Psi}$, modulo des commutateurs à symbole dans $S_{1,0}^{0}$ et le lemme 3.6, on obtient que

$$
\begin{array}{r}
\int_{0}^{T}\left\|\sqrt{\lambda}\left(\mid x-x_{\mu_{0}}\right) J^{1 / 2} \mathbf{C} u\right\|_{0}^{2} d t \leq A\left(\int_{0}^{T}|\langle\Psi \mathbf{C} f, \mathbf{C} u\rangle| d t+\sup _{0 \leq t \leq T}\|\mathbf{C} u(t)\|_{0}^{2}\right) \\
+A \sup _{\mu, i}\left\|\varphi_{i, \mu}\right\|_{C^{M}}^{3}\left(R T \sup _{0 \leq t \leq T}\|u(t)\|_{0}^{2}+\frac{1}{R}\left\|\left|J^{1 / 2} u\right|\right\|_{T}^{2}\right) .
\end{array}
$$

On obtient donc le résultat en prenant alors le sup sur $\mu_{0}$.

\subsection{Preuve du lemme 3.5}

La proposition (a) est évidente. Vérifions la proposition (b).

Lemme 4.2. Pour tout multi-indices $\alpha$ et $\beta$, il existe $A_{\alpha, \beta} \in \mathbb{R}$ tel que pour tout $\mu \in \mathbb{Z}^{n}$ et tout $R \in[1,+\infty)$,

$$
\left|\partial_{x}^{\alpha} \partial_{\xi}^{\beta} \psi\left(\frac{R\left\langle x-x_{\mu}\right\rangle}{\langle\xi\rangle}\right)\right| \leq A_{\alpha, \beta}\langle\xi\rangle^{-|\beta|} .
$$

Preuve : voir à la fin de la démonstration du lemme 3.5.

Revenons à la preuve du lemme 3.5. Les fonctions $\theta, \widetilde{\varphi}_{1, \mu}$ et $\psi$ sont $C^{\infty}$, donc $\gamma_{R, \mu}$ est $C^{\infty}$. De plus, pour tout multi-indice $\alpha$, on a

$$
\partial_{x}^{\alpha} \gamma_{R, \mu}(x, \xi)=\theta_{1}\left(\frac{|\xi|}{R}\right) \sum_{|\gamma| \leq|\alpha|} A_{\gamma, \alpha} \partial_{x}^{\gamma}\left(\psi\left(\frac{R\left\langle x-x_{\mu}\right\rangle}{\langle\xi\rangle}\right)\right) \partial_{x}^{\alpha-\gamma} v_{\mu}(x, \xi)
$$

et, pour tout multi-indice $\beta$, on a donc

$$
\begin{aligned}
& \partial_{\xi}^{\beta} \partial_{x}^{\alpha} \gamma_{R, \mu}(x, \xi) \\
& \quad=\sum_{\left|\gamma_{1}\right| \leq|\beta|,|\gamma| \leq|\alpha|} A_{\gamma, \alpha, \gamma_{1}, \beta} \partial_{\xi}^{\gamma_{1}}\left[\theta_{1}\left(\frac{|\xi|}{R}\right) \partial_{x}^{\gamma}\left(\psi\left(\frac{R\left\langle x-x_{\mu}\right\rangle}{\langle\xi\rangle}\right)\right)\right] \partial_{\xi}^{\beta-\gamma_{1}} \partial_{x}^{\alpha-\gamma} v_{\mu}(x, \xi) .
\end{aligned}
$$

Or, $\theta_{1} \in C^{\infty}(\mathbb{R})$ est une fonction plateau telle que $\theta_{1}(x)=0$ si $|x| \leq 1$ et $\theta_{1}(x)=1$ si $|x| \geq 2$ donc on a $\theta_{1}(|\xi| / R) \in S_{1,0}^{0}$ avec, sur le support de $\theta_{1}^{\prime},|\xi| \sim R$.

En utilisant les estimations obtenues sur $\eta_{\mu}$ (lemme 3.4 ), sachant que sur le support $\psi$,

$$
\left\langle x-x_{\mu}\right\rangle^{\left|\beta-\gamma_{1}\right|} \leq 2^{\left|\beta-\gamma_{1}\right|}\left(\frac{\langle\xi\rangle}{R}\right)^{\left|\beta-\gamma_{1}\right|},
$$

on en déduit donc que, pour $|\xi| \geq 1$,

$$
\begin{aligned}
\left|\partial_{\xi}^{\beta} \partial_{x}^{\alpha} \gamma_{R, \mu}(x, \xi)\right| \leq A \sup _{\left|\gamma_{1}\right|}\langle\xi\rangle^{-\left|\gamma_{1}\right|}\langle\xi\rangle^{-\left|\beta-\gamma_{1}\right|} 2^{\left|\beta-\gamma_{1}\right|}\left(\frac{\langle\xi\rangle}{R}\right)^{\left|\beta-\gamma_{1}\right|}\left\|\varphi_{1, \mu}\right\|_{C^{\left|\beta-\gamma_{1}\right|+|\alpha-\gamma|}}, \\
\text { soit }\left|\partial_{\xi}^{\beta} \partial_{x}^{\alpha} \gamma_{R, \mu}(x, \xi)\right| \leq A\langle\xi\rangle^{-|\beta|}\left\|\varphi_{1, \mu}\right\|_{C|\beta|+|\alpha|} .
\end{aligned}
$$

Le théorème de Calderón-Vaillancourt donne alors le résultat pour $M$ assez grand. Ce qui prouve la proposition (b). 
Prouvons alors la proposition (d) dans le cas $\alpha=\beta=0$. On a

$$
\begin{aligned}
& \partial_{\xi_{k}} \gamma_{R, \mu}(x, \xi)=\frac{1}{R} \frac{\xi_{k}}{|\xi|} \theta_{1}^{\prime}\left(\frac{|\xi|}{R}\right) \psi\left(\frac{R\left\langle x-x_{\mu}\right\rangle}{\langle\xi\rangle}\right) v_{\mu}(x, \xi) \\
& \quad+\theta_{1}\left(\frac{|\xi|}{R}\right) \partial_{\xi_{k}}\left(\psi\left(\frac{R\left\langle x-x_{\mu}\right\rangle}{\langle\xi\rangle}\right)\right) v_{\mu}(x, \xi)+\theta_{1}\left(\frac{|\xi|}{R}\right) \psi\left(\frac{R\left\langle x-x_{\mu}\right\rangle}{\langle\xi\rangle}\right) \partial_{\xi_{k}} v_{\mu}(x, \xi)
\end{aligned}
$$

La proposition (d) s'obtient en remarquant que $|\xi|^{-1} \leq 1 / R$ sur le support de $\theta_{1}$, que d'après le lemme $4.2, \partial_{\xi_{k}}\left(\psi\left(R\left\langle x-x_{\mu}\right\rangle /\langle\xi\rangle\right)\right) \in S_{1,0}^{-1}$ avec ses semi-normes uniformément bornées en $R$, sachant que sur le support de $\psi\left(R\left\langle x-x_{\mu}\right\rangle /\langle\xi\rangle\right)$ et de ses dérivées $\left\langle x-x_{\mu}\right\rangle \leq 2\langle\xi\rangle / R, \partial_{\xi_{k}} v_{\mu}(x, \xi) \in S_{1,0}^{0}$. Le cas $\alpha$ et $\beta$ quelconque s'obtient avec les mêmes arguments en utilisant, en plus, la formule de Faa-di-Bruno par exemple.

Prouvons ensuite la proposition (c).

En utilisant l'égalité donnée dans le lemme 3.4, vérifiée par $\eta_{\mu}$, on a

$$
-2 \widetilde{\xi} \cdot \nabla_{x} \gamma_{R, \mu}(x, \xi)-\mathscr{I}\left(\widetilde{\varphi}_{1, \mu}^{\delta}(x, \xi)\right) \cdot \xi=e_{R, \mu}(x, \xi)
$$

avec

$$
\begin{aligned}
& e_{R, \mu}(x, \xi)=-2 \frac{R \widetilde{\xi} \cdot \nabla_{x}\left(\left\langle x-x_{\mu}\right\rangle\right)}{\langle\xi\rangle} \psi^{\prime}\left(\frac{R\left\langle x-x_{\mu}\right\rangle}{\langle\xi\rangle}\right) \theta_{1}\left(\frac{|\xi|}{R}\right) v_{\mu}(x, \xi) \\
& \quad+\left(1-\psi\left(\frac{R\left\langle x-x_{\mu}\right\rangle}{\langle\xi\rangle}\right)\right) \mathscr{I}\left(\widetilde{\varphi}_{1, \mu}^{\delta}(x, \xi)\right) \cdot \xi+\left(1-\theta_{1}\left(\frac{|\xi|}{R}\right)\right) \mathscr{I}\left(\widetilde{\varphi}_{1, \mu}^{\delta}(x, \xi)\right) . \xi
\end{aligned}
$$

Pour obtenir la proposition (c), on peut remarquer que le premier terme est dans $S_{0,0}^{0}$ et que les deux derniers sont dans $S^{-\infty}$. Pour prouver cela, on utilise le support de $1-\psi, 1 \leq R\left\langle x-x_{\mu}\right\rangle /\langle\xi\rangle$ et que, pour $|\alpha|+|\beta| \neq 0, \partial_{x}^{\alpha} \partial_{\xi}^{\beta}(1-\psi)=\partial_{x}^{\alpha} \partial_{\xi}^{\beta} \psi$, ce qui permet d'appliquer le lemme 4.2 dans ce cas, sachant que $\widetilde{\varphi}_{1, \mu}$ absorbe les puissances de $\left\langle x-x_{\mu}\right\rangle$. Pour le troisième terme, on utilise le support de $1-\theta_{1}$.

Preuve du lemme 4.2. Pour simplifier, on écrit la démonstration dans le cas $\mu=0$, la cas $\mu$ quelconque se traitant exactement de la même façon.

En utilisant la formule de Fàa di Bruno, pour tout $\alpha \in \mathbb{N}^{n}$, on a

$$
\begin{aligned}
& \partial_{x}^{\alpha}\left(\psi\left(\frac{R\langle x\rangle}{\langle\xi\rangle}\right)\right)=\sum_{\substack{q \in \mathbb{N} \\
q \leq|\alpha|}} \sum_{\substack{\alpha=\nu_{1}+\cdots+\nu_{q} \\
\nu_{i} \neq 0}} \psi^{q}\left(\frac{R\langle x\rangle}{\langle\xi\rangle}\right) \partial_{x}^{\nu_{1}}(\langle x\rangle) \ldots \partial_{x}^{\nu_{q}}(\langle x\rangle)\left(\frac{R}{\langle\xi\rangle}\right)^{q}, \\
& \partial_{\xi}^{\beta} \partial_{x}^{\alpha}\left(\psi\left(\frac{R\langle x\rangle}{\langle\xi\rangle}\right)\right) \\
& \quad=\sum_{\substack{q \in \mathbb{N} \\
q \leq|\alpha|}} \sum_{\substack{\alpha=\nu_{1}+\cdots+\nu_{q} \\
\nu_{i} \neq 0}} \sum_{\gamma \leq \beta} a_{\gamma, \beta} \partial_{\xi}^{\gamma}\left(\psi^{q}\left(\frac{R\langle x\rangle}{\langle\xi\rangle}\right)\right) \partial_{x}^{\nu_{1}}(\langle x\rangle) \ldots \partial_{x}^{\nu_{q}}(\langle x\rangle) R^{q} \partial_{\xi}^{\beta-\gamma}\left(\langle\xi\rangle^{-q}\right) .
\end{aligned}
$$


On applique à nouveau la formule de Fàa di Bruno en $\xi$, ce qui donne

$$
\begin{aligned}
\partial_{\xi}^{\beta} \partial_{x}^{\alpha}\left(\psi\left(\frac{R\langle x\rangle}{\langle\xi\rangle}\right)\right) & \left.=\sum_{\substack{q \in \mathbb{N} \\
q \leq|\alpha|}} \sum_{\substack{\alpha=\nu_{1}+\ldots+\nu_{q} \\
\nu_{i} \neq 0}} \sum_{\gamma \leq \beta} a_{\gamma, \beta} \partial_{x}^{\nu_{1}}(\langle x\rangle)\right) \ldots \partial_{x}^{\nu_{q}}(\langle x\rangle) R^{q} \partial_{\xi}^{\beta-\gamma}\left(\langle\xi\rangle^{-q}\right) \\
& +\sum_{q_{2} \leq|\gamma|} \sum_{\substack{\gamma=\gamma_{1}+\cdots+\gamma_{q_{2}} \\
\gamma_{i} \neq 0}} \psi^{q+q_{2}}\left(\frac{R\langle x\rangle}{\langle\xi\rangle}\right) \partial_{\xi}^{\gamma_{1}}\left(\langle\xi\rangle^{-1}\right) \ldots \partial_{\xi}^{\gamma_{q_{2}}}\left(\langle\xi\rangle^{-1}\right)\langle x\rangle^{q_{2}} R^{q_{2}} .
\end{aligned}
$$

et

$$
\left|\partial_{\xi}^{\beta} \partial_{x}^{\alpha}\left(\psi\left(\frac{R\langle x\rangle}{\langle\xi\rangle}\right)\right)\right| \leq A_{\beta, \alpha} \sum_{q, q_{2}, \gamma}\langle x\rangle^{-|\nu|} R^{q}\langle\xi\rangle^{-q-|\beta|+|\gamma|}\langle\xi\rangle^{-q_{2}-|\gamma|}\langle x\rangle^{q_{2}} R^{q_{2}} .
$$

Sur le support de $\psi(R\langle x\rangle /\langle\xi\rangle)$ on a $(R\langle x\rangle /\langle\xi\rangle)^{q} \leq 2^{q}$ et $\langle x\rangle^{-q-|\nu|} \leq 1$, on obtient donc que

$$
\left|\partial_{\xi}^{\beta} \partial_{x}^{\alpha}\left(\psi\left(\frac{R\langle x\rangle}{\langle\xi\rangle}\right)\right)\right| \leq A_{\beta, \alpha}\langle\xi\rangle^{-|\beta|},
$$

avec $A_{\beta, \alpha}$ indépendante de $R$.

\subsection{Preuve du lemme 3.6}

Les propositions (i), (ii), (iii) découlent directement des propriétés de $\gamma_{R, \mu}$.

Pour démontrer (vi), on pose $s_{R}(x, \xi)=\exp \left(-\gamma_{R}(x, \xi)\right)$. On observe que les propriétés (i), (ii), (iii) sont encore vraies pour $s_{R}(x, \xi)$. D'après le théorème 2.1, on a

$$
\mathbf{C}(x, D) S(x, D)=I+L_{1}, \quad S(x, D) \mathbf{C}(x, D)=I+L_{2}
$$

avec $L_{1}, L_{2} \in \mathrm{Op} S_{0,0}^{0}$ et, d'aprés la proposition (d) du lemme 3.5, $\left\|L_{i}\right\|_{\mathscr{L}\left(L^{2}\right)} \leq$ $A \sup _{\mu}\left\|\varphi_{1, \mu}\right\|_{C^{M}} / R$.

Pour prouver (v), l'opérateur $\mathscr{L}$ étant différentiel, par intégrations par parties, on a

$$
i[\mathbf{C} \mathscr{L}-\mathscr{L} \mathbf{C}]=-2\left(\widetilde{\xi} \cdot \nabla_{x} \mathbf{c}\right)(x, D)+E
$$

où $E$ a son symbole $\mathscr{L}_{x} \mathbf{c}(x, \xi)$ dans $S_{0,0}^{0}$ avec ses semi-normes majorées par $A$ $\sup _{\mu}\left\|\varphi_{1, \mu}\right\|_{C^{M}}$. De plus, on a

$$
\left.-2 \widetilde{\xi} \cdot \nabla_{x} \mathbf{c}(x, \xi)=\mathbf{c}(x, \xi)\left(\mathscr{I}\left(\widetilde{b}_{1}^{\delta}(x, \xi)\right) \cdot \xi+e_{1, R}(x, \xi)\right)\right)
$$

où $e_{1, R}(x, \xi)=\sum_{\mu} \alpha_{1, \mu} e_{R, \mu}(x, \xi)$ est défini comme dans la preuve du lemme 3.5. Posons $E_{1, R}=\left(\mathbf{c} e_{1, R}\right)(x, D)$. D'après (ii), $\mathbf{c} \in S_{0,0}^{0}$ avec ses semi-normes bornées par $A \sup _{\mu}\left\|\varphi_{1, \mu}\right\|_{C^{M}}$, et $e_{1, R}$ par $A R \sup _{\mu}\left\|\varphi_{1, \mu}\right\|_{C^{M}}$, donc, d'après le théorème 2.1 et le théorème de Calderón-Vaillancourt, pour $M$ assez grand,

$$
\left\|E_{1, R}\right\|_{\mathscr{L}\left(L^{2}\left(\mathbb{R}^{n}\right)\right)} \leq A R \sup _{\mu}\left\|\varphi_{1, \mu}\right\|_{C^{M}}^{2} .
$$

On pose

$$
E_{2, R}=\mathbf{C} T_{b_{1}}^{\delta} \cdot \nabla_{x}-\left(\mathbf{c}(x, \xi) \widetilde{i b}_{1}^{\delta}(x, \xi) \cdot \xi\right)(x, D)
$$


En notant $e_{2, R}$ le symbole de $E_{2, R}$, on a $e_{2, R}(x, \xi)=\sum_{\mu} \alpha_{1, \mu} e_{2, \mu}(x, \xi)$ avec

$$
e_{2, \mu}(x, \xi)=-\sum_{|\gamma|=1} \int_{0}^{1} \int e^{i y \cdot \eta} \partial_{\xi}^{\gamma} \mathbf{c}(x, \xi+\theta \eta) \partial_{x}^{\gamma} \widetilde{\varphi}_{1, \mu}^{\delta}(x+y, \xi) \cdot \xi d y d \eta d \theta
$$

Lemme 4.3. Le symbole $e_{2, \mu}(x, \xi) \in S_{0,0}^{1}$ avec semi-normes bornées par

$$
\frac{A}{R} \sup _{\mu}\left\|\varphi_{1, \mu}\right\|_{C^{M}}^{2}
$$

De plus, $e_{2, \mu}$ absorbe les puisances de $\left\langle x-x_{\mu}\right\rangle$.

Preuve : On utilise le lemme 2.1 puisque $\partial_{\xi} \mathbf{c}, \partial_{x} \widetilde{\varphi}_{1, \mu}^{\delta} \in S_{0,0}^{0}$, associé au lemme 3.6.

On a

$$
\left.\left\langle E_{2, R} u, S u\right\rangle=\sum_{\mu} \alpha_{1, \mu}\left\langle e_{2, \mu}(x, D) J^{-1 / 2}+r(x, D)\right) u, J^{1 / 2} S u\right\rangle
$$

où, d'après le théorème $2.1, r(x, D) \in O p S_{0,0}^{-1 / 2}$ avec

$$
\left\|J^{1 / 2} r(x, D)\right\|_{\mathscr{L}\left(L^{2}\right)} \leq A \sup _{\mu}\left\|\varphi_{1, \mu}\right\|_{C^{M}}^{2},
$$

donc

$$
\left|\left\langle r(x, D) u, J^{1 / 2} S u\right\rangle\right| \leq A \sup _{\mu}\left\|\varphi_{1, \mu}\right\|_{C^{M}}^{2}\|S\|_{\mathscr{L}\left(L^{2}\right)}\|u\|_{0}^{2}
$$

car $S \in O p S_{0,0}^{0}$.

Si $S=\mathbf{C},\|S\|_{\mathscr{L}\left(L^{2}\right)} \leq A \sup _{\mu}\left\|\varphi_{1, \mu}\right\|_{C^{M}}$. De plus

$$
\left\langle e_{2, \mu}(x, D) J^{-1 / 2} u, J^{1 / 2} S u\right\rangle=\left\langle\left\langle x-x_{\mu}\right\rangle^{2} e_{2, \mu}(x, D) J^{-1 / 2} u,\left\langle x-x_{\mu}\right\rangle^{-2} J^{1 / 2} S u\right\rangle
$$

et

$$
\left\langle x-x_{\mu}\right\rangle^{2} e_{2, \mu}(x, D) J^{-1 / 2} u=\left\langle x-x_{\mu}\right\rangle^{2} e_{2, \mu}(x, D) J^{-1}\left\langle x-x_{\mu}\right\rangle^{2}\left\langle x-x_{\mu}\right\rangle^{-2} J^{1 / 2} u .
$$

Sachant que $\left\langle x-x_{\mu}\right\rangle^{2}$ est un polynôme de degré 2 , on a

$$
\begin{aligned}
\left\langle x-x_{\mu}\right\rangle^{2} & e_{2, \mu}(x, D) J^{-1}\left\langle x-x_{\mu}\right\rangle^{2} \\
= & \left(\left\langle x-x_{\mu}\right\rangle^{4} e_{2, \mu}(x, \xi)\langle\xi\rangle^{-1}\right)(x, D) \\
& +\left(\left\langle x-x_{\mu}\right\rangle^{2} \nabla_{\xi}\left(e_{2, \mu}(x, \xi)\langle\xi\rangle^{-1}\right) \cdot \nabla_{x}\left(\left\langle x-x_{\mu}\right\rangle^{2}\right)\right)(x, D) \\
& +\sum_{|\nu|=2}\left(\left\langle x-x_{\mu}\right\rangle^{2} \partial_{\xi}^{\nu}\left(\langle\xi\rangle^{-1} e_{2, \mu}(x, \xi)\right) \partial_{x}^{\nu}\left(\left\langle x-x_{\mu}\right\rangle^{2}\right)\right)(x, D) .
\end{aligned}
$$

En utilisant le lemme 4.3, sachant que $\sum_{\mu}\left|\alpha_{1, \mu}\right| \leq A_{1}$, on obtient donc que

$$
\begin{aligned}
& \left|\int_{0}^{T}\left\langle E_{2, R} u, S u\right\rangle d t\right| \\
& \quad \leq \frac{A \sup _{\mu}\left\|\varphi_{1, \mu}\right\|_{C^{M}}^{2} A_{1}}{R}\left\|\left|J^{1 / 2} S u\left\|\left.\right|_{T}+A \sup _{\mu}\right\| \varphi_{1, \mu}\left\|_{C^{M}}^{2}\right\| S\left\|_{\mathscr{L}\left(L^{2}\right)} T \sup _{[0, T]}\right\| u \|_{0}^{2} .\right.\right.
\end{aligned}
$$


En remarquant que $J^{1 / 2} S=S J^{1 / 2}+\left[J^{1 / 2}, S\right]$ avec $\left[J^{1 / 2}, S\right] \in S_{0,0}^{0}$ et en utilisant le corollaire 2.1, on obtient l'estimation annoncée pour $E_{2, R}$.

Preuve de la proposition (iv). On remarque que

$$
\left[\mathbf{C}, T_{b_{i}}^{\delta} \cdot \nabla\right]=E_{2, R}+\left(\widetilde{b}_{i}^{\delta}(x, \xi) \cdot i \xi \mathbf{c}(x, \xi)\right)(x, D)-T_{b_{i}}^{\delta} \cdot \nabla \mathbf{C}
$$

Pour le second terme, on applique la même démonstration que pour $E_{2, R}$ sachant que $T_{b_{i}}^{\delta} \in O p S_{1, \delta}^{0, \varrho}$.

C'est à dire que si l'on dérive en $\xi$ et que l'on ne dépasse pas $\varrho$, on gagne 1 en $|\xi|$. Si on dépasse $\varrho$, on utilise que $T_{b_{i}}^{\delta} \in O p S_{0,0}^{0}$ avec $\left\|T_{b_{i}}^{\delta}\right\| \mathscr{L}\left(L^{2}\right) \leq A \sup _{\mu}\left\|\varphi_{1, \mu}\right\|_{C^{M}}$.

\subsection{Preuve du lemme 3.8}

On a

$$
2 \mathscr{R}\left\langle\mathbf{C} T_{\mathscr{R}\left(b_{1}\right)} \cdot \nabla u, \mathbf{C} u\right\rangle=\left\langle\mathbf{C} T_{\mathscr{R}\left(b_{1}\right)} \cdot \nabla u, \mathbf{C} u\right\rangle+\left\langle\mathbf{C} u, \mathbf{C} T_{\mathscr{R}\left(b_{1}\right)} \cdot \nabla u\right\rangle
$$

or,

$$
\left\langle\mathbf{C} u, \mathbf{C} T_{\mathscr{R}\left(b_{1}\right)} \cdot \nabla u\right\rangle=\left\langle\mathbf{C} u,\left[\mathbf{C}, T_{\mathscr{R}\left(b_{1}\right)} \cdot \nabla\right] u\right\rangle+\left\langle\left(T_{\mathscr{R}\left(b_{1}\right)} \cdot \nabla\right)^{*} \mathbf{C} u, \mathbf{C} u\right\rangle
$$

et, d'après le théorème 2.1 ,

$$
T_{\mathscr{R}\left(b_{1}\right)} \cdot \nabla=\left(\mathscr{R}\left(\widetilde{b}_{1}(x, \xi)\right) \cdot i \xi\right)(x, D)
$$

et

$$
\begin{aligned}
\left(T_{\mathscr{R}\left(b_{1}\right)} \cdot \nabla\right)^{*} & =\left(\mathscr{R}\left(\widetilde{b}_{1}(x, \xi)\right) \cdot i \xi\right)(x, D)^{*} \cdot\left(\mathscr{R}\left(\widetilde{b}_{1}(x, \xi)\right) \cdot i \xi\right)(x, D)^{*} \\
& =-\left(\mathscr{R}\left(\widetilde{b}_{1}(x, \xi)\right) \cdot i \xi\right)(x, D)+r_{1}(x, D)
\end{aligned}
$$

et donc

$$
\left(T_{\mathscr{R}\left(b_{1}\right)} \cdot \nabla\right)^{*}=-T_{\mathscr{R}\left(b_{1}\right)} \cdot \nabla+r_{1}(x, D)
$$

avec $r_{1} \in S_{1, \delta}^{0}$ si $\varrho \geq 1$ avec ses semi-normes majorées par des semi-normes de $\widetilde{b}_{1}$ qui, elles-mêmes, sont majorées par Cste $\cdot \sup _{\mu}\left\|\varphi_{1, \mu}\right\|_{C^{M}}$ pour $M$ assez grand. On obtient donc que

$$
\begin{aligned}
2 \mathscr{R}\left\langle\mathbf{C} T_{\mathscr{R}\left(b_{1}\right)} \cdot \nabla u, \mathbf{C} u\right\rangle= & \left\langle\mathbf{C} T_{\mathscr{R}\left(b_{1}\right)} \cdot \nabla u, \mathbf{C} u\right\rangle-\left\langle T_{\mathscr{R}\left(b_{1}\right)} \cdot \nabla \mathbf{C} u, \mathbf{C} u\right\rangle \\
& +\left\langle r_{1}(x, D) \mathbf{C} u, \mathbf{C} u\right\rangle+\left\langle\mathbf{C} u,\left[\mathbf{C}, T_{\mathscr{R}\left(b_{1}\right)} \cdot \nabla\right] u\right\rangle \\
2 \mathscr{R}\left\langle\mathbf{C} T_{\mathscr{R}\left(b_{1}\right)} \cdot \nabla u, \mathbf{C} u\right\rangle= & \left\langle\left[\mathbf{C}, T_{\mathscr{R}\left(b_{1}\right)} \cdot \nabla\right] u, \mathbf{C} u\right\rangle+\left\langle r_{1}(x, D) \mathbf{C} u, \mathbf{C} u\right\rangle \\
& +\left\langle\mathbf{C} u,\left[\mathbf{C}, T_{\mathscr{R}\left(b_{1}\right.} \cdot \nabla\right] u\right\rangle .
\end{aligned}
$$

On intègre alors sur $[0, T]$ et, les propositions (ii), (iii) et (iv) du lemme 3.6 donnent l'estimation souhaitée car $\mathscr{R}\left(b_{1}\right)$ a les mêmes propriétés que $b_{1}$.

\subsection{Preuve du lemme 3.7}

On a

$$
\left\langle\mathbf{C} T_{b_{2}}^{\delta} \cdot \nabla_{x} \bar{u}, \mathbf{C} u\right\rangle=\left\langle\left[\mathbf{C}, T_{b_{2}}^{\delta} \cdot \nabla_{x}\right] \bar{u}, \mathbf{C} u\right\rangle+\left\langle T_{b_{2}}^{\delta} \cdot \nabla_{x} \mathbf{C} \bar{u}, \mathbf{C} u\right\rangle
$$


or, d'après la proposition (iv) du lemme 3.6, on a

$$
\begin{aligned}
& \left|\int_{0}^{T}\left\langle\left[\mathbf{C}, T_{b_{2}}^{\delta} \cdot \nabla_{x}\right] \bar{u}, \mathbf{C} u\right\rangle d t\right| \\
& \quad \leq A \sup _{\mu}\left(\left\|\varphi_{1, \mu}\right\|_{C^{M}}^{2}\left\|\varphi_{2, \mu}\right\|_{C^{M}}\right)\left(\frac{1}{R}\|\| J^{1 / 2} u\|\|_{T}^{2}+R T \sup _{[0, T]}\|u\|_{0}^{2}\right) .
\end{aligned}
$$

De plus, $\mathbf{c}(x, \xi)$ est réel et pair en $\xi$ donc $\mathbf{C} \bar{u}=\overline{\mathbf{C} u}$. Le deuxième terme de cette somme est donc de la forme $\left\langle T_{b_{2}}^{\delta} \cdot \nabla_{x} \bar{v}, v\right\rangle$ avec $v=\mathbf{C} u$.

On a

$$
\left\langle T_{b_{2}}^{\delta} \cdot \nabla_{x} \bar{v}, v\right\rangle=-\left\langle\bar{v}, \nabla_{x} \cdot\left(T_{\bar{b}_{2}}^{\delta}+r(x, D)\right) v\right\rangle \quad \text { avec } r \in S_{1, \delta}^{-1, \varrho-1} .
$$

Le terme en $\nabla_{x} \cdot r(x, D)$ étant d'ordre 0 pour $\varrho \geq 1$, il ne pose pas de problème et ne sera donc pas écrit dans la suite. On a alors $\left\langle T_{b_{2}}^{\delta} \cdot \nabla_{x} \bar{v}, v\right\rangle=-\overline{\left\langle\nabla_{x} \cdot T_{b_{2}}^{\delta} v, \bar{v}\right\rangle}$ or $\overline{\nabla_{x} v}=\nabla_{x} \bar{v}$, sachant que $\widetilde{b}_{2}(x, \xi)$ est pair en $\xi$, on a $\overline{T_{\bar{b}_{2}} v}=T_{b_{2}} \bar{v}$, et $\left\langle T_{b_{2}}^{\delta} . \nabla_{x} \bar{v}, v\right\rangle=$ $-\left\langle\nabla_{x} \cdot T_{b_{2}}^{\delta} \bar{v}, v\right\rangle$ or $\nabla_{x} \cdot T_{b_{2}}^{\delta}=T_{b_{2}}^{\delta} \cdot \nabla_{x}+r_{1}(x, D)$ avec $r_{1} \in S_{1, \delta}^{0, \varrho-1}$ donc

$$
2\left\langle T_{b_{2}}^{\delta} \cdot \nabla_{x} \bar{v}, v\right\rangle=\left\langle\left(r+r_{1}\right)(x, D) \bar{v}, v\right\rangle \leq A\|\mathbf{C} u\|_{0}^{2} \leq A \sup _{\mu, i}\left\|\varphi_{i, \mu}\right\|_{C^{M}}^{3} \sup _{[0, T]}\|u\|_{0}^{2} .
$$

Ce qui termine la preuve du lemme 3.7.

\subsection{Preuve du lemme 3.9}

Soit $m^{\prime}$ un nombre réel positif fixé assez grand. On commence par écrire que

$$
T_{\varphi_{i, \mu}}^{\delta}-T_{\varphi_{i, \mu, m}}^{\delta}=T_{\varphi_{i, \mu}}^{\delta}-T_{\varphi_{i, \mu, m^{\prime}}}^{\delta}+T_{b}
$$

o ù $\forall x \in \mathbb{R}^{n}, b(x)=\varphi_{i, \mu, m^{\prime}}(x)-\varphi_{i, \mu, m}(x)$. On a

$$
\left\langle\mathbf{C}_{m} T_{b} . \nabla u, \mathbf{C}_{m} u\right\rangle=\left\langle T_{b} . \nabla \mathbf{C}_{m} u, \mathbf{C}_{m} u\right\rangle+\left\langle\left[\mathbf{C}_{m}, T_{b} . \nabla\right] u, \mathbf{C}_{m} u\right\rangle .
$$

Comme dans la preuve de la proposition (iv) du lemme 3.6, on a

$$
\left|\int_{0}^{T}\left\langle\left[\mathbf{C}_{m}, T_{b} . \nabla\right] u, \mathbf{C}_{m} u\right\rangle d t\right| \leq A \sup _{\mu, i}\left\|\varphi_{i, \mu}\right\|_{C^{M}}^{3}\left(R T \sup _{[0, T]}\|u\|_{0}^{2}+\frac{1}{R}\|\| J^{1 / 2} u\|\|_{T}^{2}\right) .
$$

De plus, on a

$$
\begin{aligned}
& \left\langle T_{b} \cdot \nabla \mathbf{C}_{m} u, \mathbf{C}_{m} u\right\rangle=\left\langle J^{-1 / 2} T_{b} \cdot \nabla \mathbf{C}_{m} u, J^{1 / 2} \mathbf{C}_{m} u\right\rangle \\
& =\left\langle\left[J^{-1 / 2}, T_{b} \cdot \nabla\right] \mathbf{C}_{m} u, J^{1 / 2} \mathbf{C}_{m} u\right\rangle+\left\langle\left\langle x-x_{\mu}\right\rangle^{2} T_{b} \cdot \nabla J^{-1 / 2} \mathbf{C}_{m} u,\left\langle x-x_{\mu}\right\rangle^{-2} J^{1 / 2} \mathbf{C}_{m} u\right\rangle .
\end{aligned}
$$

D'après le théorème 2.1 , pour $\varrho \geq 1,\left[J^{-1 / 2}, T_{b} . \nabla\right] \in O p S_{1, \delta}^{-1 / 2}$ avec les semi-normes de son symbole majorées par $A\|b\|_{C^{1}}$. On a donc

$$
\int_{0}^{T}\left\langle\left[J^{-1 / 2}, T_{b} \cdot \nabla\right] \mathbf{C}_{m} u, J^{1 / 2} \mathbf{C}_{m} u\right\rangle d t \leq A T\|b\|_{C^{1}}\left\|\mathbf{C}_{m}\right\|_{\mathscr{L}\left(L^{2}\right)}\|u\|_{0}^{2} .
$$


De plus, on a

$$
\begin{aligned}
& \left\langle\left\langle x-x_{\mu}\right\rangle^{2} T_{b} . \nabla J^{-1 / 2} \mathbf{C}_{m} u,\left\langle x-x_{\mu}\right\rangle^{-2} J^{1 / 2} \mathbf{C}_{m} u\right\rangle \\
& \quad=\left\langle\left\langle x-x_{\mu}\right\rangle^{2} T_{b} . \nabla J^{-1}\left\langle x-x_{\mu}\right\rangle^{2}\left\langle x-x_{\mu}\right\rangle^{-2} J^{1 / 2} \mathbf{C}_{m} u,\left\langle x-x_{\mu}\right\rangle^{-2} J^{1 / 2} \mathbf{C}_{m} u\right\rangle,
\end{aligned}
$$

or

$$
\left\langle x-x_{\mu}\right\rangle^{2} T_{b} . \nabla J^{-1}\left\langle x-x_{\mu}\right\rangle^{2} \in O p S_{1, \delta}^{0}
$$

avec ses semi-normes majorées par $\|b\|_{L^{\infty}}$. En effet, le symbole de cet opérateur est

$$
\left\langle x-x_{\mu}\right\rangle^{2} \sum_{|\nu| \leq 2} \partial_{\xi}^{\nu}\left(\widetilde{b}(x, \xi) i \xi\langle\xi\rangle^{-1}\right)\left(-i \partial_{x}\right)^{\nu}\left(\left\langle x-x_{\mu}\right\rangle^{2}\right) .
$$

Sachant que $\widetilde{b}$ absorbe les puissance $\left\langle x-x_{\mu}\right\rangle$, on obtient le résultat. On a donc

$$
\begin{aligned}
\int_{0}^{T}\left\langle\left\langle x-x_{\mu}\right\rangle^{2} T_{b} . \nabla J^{-1 / 2} \mathbf{C}_{m} u,\left\langle x-x_{\mu}\right\rangle^{-2}\right. & \left.J^{1 / 2} \mathbf{C}_{m} u\right\rangle d t \\
& \leq A\|b\|_{L^{\infty}} \mid\left\|J^{1 / 2} \mathbf{C}_{m} u\right\| \|_{T}^{2},
\end{aligned}
$$

or, d'après le lemme 2.20 , on a $\|b\|_{L^{\infty}} \leq\left(A / m+A / m^{\prime}\right)\left\|\varphi_{1, \mu}\right\|_{C^{1}}$. De plus, pour le terme en $T_{\varphi_{i, \mu}}^{\delta}-T_{\varphi_{i, \mu, m^{\prime}}}^{\delta}$, on utilise $C_{m}^{*}$ pour éviter la commutation entre $C_{m}$ et $T_{\varphi_{i, \mu}}^{\delta}-T_{\varphi_{i, \mu, m^{\prime}}}^{\delta}$. C'est l'estimation de ce terme qui fait appraître $\frac{A m^{N M}}{m^{\prime}} \mid\left\|J^{1 / 2} u\right\|_{T}$ où $N$ et $M$ sont des entiers fixés assez grands ne dépendant que de $n$. En utilisant (4.7), (4.8) et (4.9), on obtient l'inégalité du lemme 3.9.

\subsection{Preuve du lemme 3.10}

L'inégalité (3.17) se prouve en utilisant l'inégalité de Cauchy-Schwarz et le fait que, pour tout réel $a$, tout réel $b$ et tout $R^{\prime}>0$,

$$
a b \leq \frac{1}{2}\left(\frac{a^{2}}{R^{\prime}}+R^{\prime} b^{2}\right) .
$$

Pour prouver l'inégalité (3.18), on commence par intercaller $\theta_{1}$. On a

$$
I_{T}\left(J^{s} f, J^{s} u\right) \leq I_{T}\left(\theta_{1}(D / R) J^{s} f, J^{s} u\right)+I_{T}\left(\left(1-\theta_{1}(D / R)\right) J^{s} f, J^{s} u\right) .
$$

Pour le deuxième terme, on utilise (3.17). Pour le premier, sachant que $\theta_{1}(D / R)$ et $J^{s}$ commutent, on a

$$
I_{T}\left(\theta_{1}(D / R) J^{s} f, J^{s} u\right) \leq \sum_{\mu}\left|\alpha_{\mu}\right| I_{T}\left(J^{s} \theta_{1}(D / R) f_{\mu}, J^{s} u\right),
$$

or

$$
I_{T}\left(J^{s} \theta_{1}(D / R) f_{\mu}, J^{s} u\right)=I_{T}\left(\left\langle x-x_{\mu}\right\rangle^{2} J^{s-1 / 2} \theta_{1}(D / R) f_{\mu},\left\langle x-x_{\mu}\right\rangle^{-2} J^{s+1 / 2} u\right) .
$$

On utilise ensuite l'inégalité de Cauchy-Schwarz, puis (4.10), en enfin, on prend le sup sur $\mu$, ce qui donne l'estimation (3.18). 


\subsection{Preuve du lemme 3.11}

On étudie tout d'abord $\left[\left\langle x-x_{\mu}\right\rangle^{2}, J^{s-1 / 2}\right]$. Le multiplicateur $\left\langle x-x_{\mu}\right\rangle^{2}$ étant un polynôme de degré 2 , on a $\left[\left\langle x-x_{\mu}\right\rangle^{2}, J^{s-1 / 2}\right]=r(x, D)$ avec

$$
r(x, \xi)=-\sum_{|\nu| \in\{1,2\}} r_{\nu}(x, \xi)
$$

où $r_{\nu}(x, \xi)=\partial_{\xi}^{\nu}\left(\langle\xi\rangle^{s-1 / 2}\right) D_{x}^{\nu}\left(\left\langle x-x_{\mu}\right\rangle^{2}\right)$ et $r_{\nu}(x, D)\left\langle x-x_{\mu}\right\rangle^{-2} \in S_{1,0}^{s-1-1 / 2}$.

En posant $b=\int_{0}^{1}\left(\partial_{t} \widetilde{\widetilde{\varphi}}_{1, \mu}\right)\left(x, t t^{\prime}\right) d t^{\prime}$, on obtient donc que

$$
\begin{aligned}
& \sup _{\mu}\left(\int_{0}^{T}\left\|\left\langle x-x_{\mu}\right\rangle^{2} J^{s-1 / 2} T_{b} \cdot \nabla w\right\|_{0}^{2} d t\right)^{1 / 2} \\
& \quad \leq A T \sup _{[0, T]}\left\|\left\langle x-x_{\mu}\right\rangle^{2} T_{b} \cdot \nabla w\right\|_{s-3 / 2}+\sup _{\mu}\left(\int_{0}^{T}\left\|\left\langle x-x_{\mu}\right\rangle^{2} T_{b} \cdot \nabla w\right\|_{s-1 / 2}^{2} d t\right)^{1 / 2} .
\end{aligned}
$$

Or, $\left\langle x-x_{\mu}\right\rangle^{2} T_{b}\left\langle x-x_{\mu}\right\rangle^{2} \in O p S_{1,1}^{0}$ car, par construction $b$ absorbe les puissances de $\left\langle x-x_{\mu}\right\rangle$ et $b \in S_{1,1}^{0}$. On obtient donc

$$
\begin{aligned}
\sup _{\mu}( & \left.\int_{0}^{T}\left\|\left\langle x-x_{\mu}\right\rangle^{2} T_{b} \cdot \nabla w\right\|_{s-1 / 2}^{2} d t\right)^{1 / 2} \\
& \leq A\left(\sup _{\mu}\left(\int_{0}^{T}\left\|\left\langle x-x_{\mu}\right\rangle^{-2} \nabla w\right\|_{s-1 / 2}^{2} d t\right)^{1 / 2}+T \sup _{[0, T]}\|w\|_{s}\right) .
\end{aligned}
$$

Après commutation de $J^{s-1 / 2}$ et $\left\langle x-x_{\mu}\right\rangle^{-2}$, on obtient l'estimation annoncée.

\subsection{Preuve du lemme 3.12}

On a

$$
\begin{aligned}
& \left\langle x-x_{\mu}\right\rangle^{2} J^{s-1 / 2} \theta_{1}\left(\frac{D}{R}\right)\left(T_{\varphi_{1, \mu}}-T_{\varphi_{1, \mu}}^{\delta}\right) . \nabla \\
& \quad=\left(\left\langle x-x_{\mu}\right\rangle^{2}\langle\xi\rangle^{s-1 / 2} \theta_{1}\left(\frac{\xi}{R}\right)\right)(x, D)\left\langle x-x_{\mu}\right\rangle^{-2}\left\langle x-x_{\mu}\right\rangle^{2}\left(T_{\varphi_{1, \mu}}-T_{\varphi_{1, \mu}}^{\delta}\right) . \nabla .
\end{aligned}
$$

En utilisant que sur le support de $\theta_{1}, R^{\delta}\langle\xi\rangle^{-\delta} \leq 1$, on a

$$
\left(\left\langle x-x_{\mu}\right\rangle^{2}\langle\xi\rangle^{s-1 / 2} \theta_{1}\left(\frac{\xi}{R}\right)\right)(x, D)\left\langle x-x_{\mu}\right\rangle^{-2} R^{\delta} J^{-\delta} \in O p S_{1, \delta}^{s-1 / 2}
$$

avec ses seminormes indépendantes de $R$. On obtient donc que

$$
\begin{aligned}
\|\left(\left\langle x-x_{\mu}\right\rangle^{2}\langle\xi\rangle^{s-1 / 2} \theta_{1}\left(\frac{\xi}{R}\right)\right)(x, D) & \left(T_{\varphi_{1, \mu}}-T_{\varphi_{1, \mu}}^{\delta}\right) . \nabla w \|_{0} \\
& \leq \frac{A}{R^{\delta}}\left\|J^{\delta}\left\langle x-x_{\mu}\right\rangle^{2}\left(T_{\varphi_{1, \mu}}-T_{\varphi_{1, \mu}}^{\delta}\right) . \nabla w\right\|_{s-1 / 2}
\end{aligned}
$$

en intercalant $\left\langle x-x_{\mu}\right\rangle^{-2} R^{\delta} J^{-\delta} R^{-\delta} J^{\delta}\left\langle x-x_{\mu}\right\rangle^{2}$. Or, pour tout $k \in \llbracket 1, n \rrbracket$, l'opérateur $\left\langle x-x_{\mu}\right\rangle^{2}\left(T_{\varphi_{1, \mu, k}}-T_{\varphi_{1, \mu, k}}^{\delta}\right)\left\langle x-x_{\mu}\right\rangle^{2}$ est borné de $H^{s}$ dans $H^{s+\delta \min (1, \varrho)} \operatorname{car}\left\langle x-x_{\mu}\right\rangle^{2}$ est un polynôme de degré 2 et donc, le symbole de cet opérateur est

$$
-\sum_{|\nu| \leq 2}\left\langle x-x_{\mu}\right\rangle^{2} \partial_{\xi}^{\nu}\left(\left(\widetilde{\varphi}_{1, \mu, k}(x, \xi)-\widetilde{\varphi}_{1, \mu, k}^{\delta}(x, \xi)\right)\right) D_{x}^{\nu}\left(\left\langle x-x_{\mu}\right\rangle^{2}\right) .
$$

De plus, on a le lemme suivant (prouver à la fin de cette démonstration), 
Lemme 4.4. Pour tout $k$ et tout $k^{\prime} \in \llbracket 1, n \rrbracket$, on a $\left.\left\langle x-x_{\mu}\right\rangle^{2}\left(\widetilde{\varphi}_{1, \mu, k}-\widetilde{\varphi}_{1, \mu, k}^{\delta}\right)\right)(x, D)$ et $\left(\left\langle x-x_{\mu}\right\rangle^{2}\left(x_{k^{\prime}}-x_{\mu, k^{\prime}}\right)\left(\widetilde{\varphi}_{1, \mu, k}-\widetilde{\varphi}_{1, \mu, k}^{\delta}\right)(x, D)\right)$ sont des opérateurs bornés de $H^{s}$ dans $H^{s+\delta \min (1, \varrho)}$.

Sachant que $\varrho>2$, on obtient donc

$\int_{0}^{T}\left\|J^{\delta}\left\langle x-x_{\mu}\right\rangle^{2}\left(T_{\varphi_{1, \mu}}-T_{\varphi_{1, \mu}}^{\delta}\right) . \nabla w\right\|_{s-1 / 2} d t \leq A \sum_{k} \int_{0}^{T}\left\|\left\langle x-x_{\mu}\right\rangle^{-2} \partial_{x_{k}} w\right\|_{s-1 / 2} d t$.

Le commutateur $\left[J^{s-1 / 2},\left\langle x-x_{\mu}\right\rangle^{-2}\right] \in S_{1,0}^{s-3 / 2}$ donc on a

$\left(\int_{0}^{T}\left\|J^{s-1 / 2}\left\langle x-x_{\mu}\right\rangle^{2} \theta_{1}\left(\frac{D}{R}\right)\left(T_{\varphi_{1, \mu}}-T_{\varphi_{1, \mu}}^{\delta}\right) . \nabla w\right\|_{0}^{2} d t\right)^{1 / 2} \leq \frac{A}{R^{\delta}} \lambda_{2}^{T}(w)+A T \lambda_{1}^{T}(w)$.

Preuve du lemme 4.4. On remarque tout d'abord que $\left\langle x-x_{\mu}\right\rangle^{2}=1+\sum_{k^{\prime}}\left(x_{k^{\prime}}-\right.$ $\left.x_{\mu, k^{\prime}}\right)^{2}$ et donc

$$
\begin{aligned}
\langle x & \left.-x_{\mu}\right\rangle^{2}\left(\widetilde{\varphi}_{1, \mu, k}(x, \xi)-\widetilde{\varphi}_{1, \mu, k}^{\delta}(x, \xi)\right) \\
& =\widetilde{\varphi}_{1, \mu, k}(x, \xi)-\widetilde{\varphi}_{1, \mu, k}^{\delta}(x, \xi)+\sum_{k^{\prime}}\left(x_{k^{\prime}}-x_{\mu, k^{\prime}}\right)^{2}\left(\widetilde{\varphi}_{1, \mu, k}(x, \xi)-\widetilde{\varphi}_{1, \mu, k}^{\delta}(x, \xi)\right) .
\end{aligned}
$$

La proposition 2.16 donne le résultat pour le premier terme. Pour l'autre terme, sachant que

$$
\left(x_{k^{\prime}}-x_{\mu, k^{\prime}}\right)^{2}=\left(x_{k^{\prime}}-y_{k^{\prime}}\right)^{2}+2\left(x_{k^{\prime}}-y_{k^{\prime}}\right)\left(y_{k^{\prime}}-x_{\mu, k^{\prime}}\right)+\left(y_{k^{\prime}}-x_{\mu, k^{\prime}}\right)^{2},
$$

que $\left|x-x_{\mu}\right|^{2}$, et que $T_{\varphi}^{\delta}=\varphi^{\delta}(x, D)$ avec

$$
\widetilde{\varphi}^{\delta}(x, \xi)=\left(1-\theta_{1}(\xi)\right)|\xi|^{\delta n} \int_{\mathbb{R}^{n}} \widehat{\chi}_{1}\left(|\xi|^{\delta}(x-y)\right) \varphi(y) d y
$$

où $\widehat{\chi}_{1} \in \mathscr{S}$, donc, pour tout $\delta \in[0,1]$, on a $\left(x_{k^{\prime}}-y_{k^{\prime}}\right)^{k_{0}} T_{\varphi}^{\delta} \in O p S_{1, \delta}^{-k_{0} \delta}$.

On a

$$
\left(x_{k^{\prime}}-x_{\mu, k^{\prime}}\right)^{2}\left(T_{\varphi_{1, \mu, k}}-T_{\varphi_{1, \mu, k}}^{\delta}\right)=\sum_{k_{0}=0}^{2}\left(\begin{array}{c}
2 \\
k_{0}
\end{array}\right)\left(x_{k^{\prime}}-y_{k^{\prime}}\right)^{2-k_{0}}\left(T_{\varphi_{k_{0}}}-T_{\varphi_{k_{0}}}^{\delta}\right)
$$

où $\varphi_{k_{0}}(y)=\left(y_{k^{\prime}}-x_{\mu, k^{\prime}}\right)^{k_{0}} \varphi_{1, \mu, k}(y) \in C^{\varrho}$. En développant le facteur $\left(x_{k^{\prime}}-y_{k^{\prime}}\right)^{2-k_{0}}$ on obtient donc que les deux premiers termes de cette somme sont dans $S_{1,1}^{-\left(2-k_{0}\right) \delta}$ car $0<\delta \leq 1$. Le troisième vérifie le lemme 2.13 et la proposition 2.16 , on obtient donc le lemme 4.4 dans la premier cas.

Le deuxième cas énoncé dans le lemme 4.4 se traite comme ci-dessus. 


\section{Références}

[1] Bony, J.-M.: Calcul symbolique et propagation des singularités pour les équations aux dérivées partielles non linéaires. Ann. Sci. École Norm. Sup. (4) 14 (1981), no. 2, 209-246.

[2] Coifman, R. et Meyer, Y.: Au delà des opérateurs pseudo-différentiels. Astérisque 57, Société Mathématique de France, Paris, 1978.

[3] Doi, S.: On the Cauchy problem for Schrödinger type equations and the regularity of the solutions. J. Math. Kyoto Univ. 34 (1994), 319-328.

[4] Kenig, C. E.: The Cauchy problem for quasi-linear Schrödinger equations (Following Kenig-Ponce-Vega). IAS/Park City Mathematics Series, 2002.

[5] Kenig, C. E., Ponce, G. And Vega, L.: Smoothing effect and local existence theory for the generalized nonlinear Schrödinger equations. Invent. Math. 134 (1998), no. 3, 489-545.

[6] Kenig, C. E., Ponce, G. And Vega, L.: Small solutions to nonlinear Schrödiger equations. Ann. Inst. H. Poincaré Anal. Non Linéaire 10 (1993), 255-288.

[7] Kenig, C.E., Ponce, G. And Vega, L.: The Cauchy problem for quasi-linear Schrödinger equations. Invent. math. 158 (2004), 343-388.

[8] Meyer, Y.: Nouvelles estimations pour les solutions d'équations aux dérivées partielles. In Séminaire Goulaoudic-Meyer-Schwartz, 1981/1982., Exp. no. VI, 13 pp. École Polytech., Palaiseau, 1982.

[9] Meyer, Y.: Remarques sur un théorème de J.-M. Bony. Rend. Circ. Mat. Palermo (2) 1981, suppl. 1, 1-20.

[10] Mizonata, S.: On the Cauchy problem. Notes and Reports in Math. in Science and Engineering 3, Academic Press, Orlando, FL; Science Press, Beijing, 1985.

[11] Ponce, G.: Local existence theory for the generalized Schrödinger equation. In Journées "Équations aux Dérivées Partielles" (Saint-Jean-de-Monts, 1997), Exp. no. XIV, 1-11. École Polytech., Palaiseau, 1997.

[12] Szeftel, J.: Microlocal dispersive smoothing for the nonlinear Schrödinger equation. SIAM J. Math. Anal. 37 (2005), no. 2, 549-597.

[13] T'Joen, L.: Effets régularisants et existence locale pour l'équation de Schrödinger non linéaire à coefficients variables. Comm. Partial Differential Equations 27 (2002), no. 3-4, 527-564.

[14] TaYlor, M. E.: Pseudodifferential operators and nonlinear PDE. Progress in Mathematics 100, Birkhäuser Boston, Boston, MA, 1991.

[15] Tataru, D.: On the Fefferman-Phong inequality and related problems. Comm. Partial Differential Equations 27 (2002), no. 11, 2101-2138.

[16] TAKeuCHI, J.: On the Cauchy problem for some non-Kowalewskian equations with distinct characteristic roots. J. Math. Kyoto Univ. 20 (1980), 105-124.

Received May 10, 2012; revised January 18, 2014.

Pierre-Yves Bienaimé : 8 Place Louis Descars, 72240 Saint Symphorien, France.

E-mail: pierre-yves.bienaime@univ-nantes.fr 